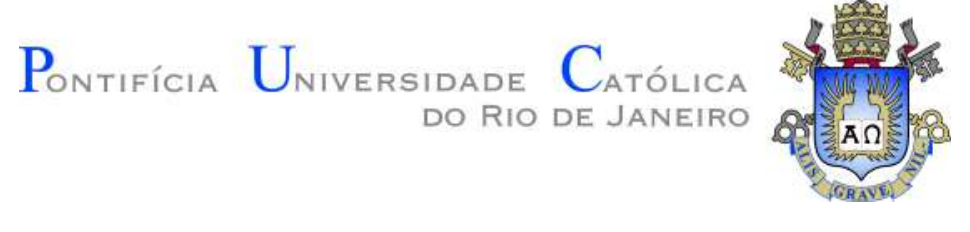

Bruna Mascotte Oliveira de Menezes

O impacto de choques no preço do petróleo: pequeno modelo para a economia brasileira

Dissertação de Mestrado

Dissertação apresentada como requisito parcial para a obtenção do grau de Mestre pelo Programa de Pós-graduação em Macroeconomia e Finanças da Pontifícia Universidade Católica do Rio de Janeiro (PUC-Rio).

Orientador: Prof. Carlos Viana de Carvalho 
Bruna Mascotte Oliveira de Menezes

\section{O impacto de choques no preço do petróleo: pequeno modelo para a economia brasileira}

Dissertação apresentada como requisito parcial para a obtenção do grau de Mestre pelo Programa de Pós-graduação em Macroeconomia e Finanças da Pontifícia Universidade Católica do Rio de Janeiro (PUC-Rio). Aprovada pela Comissão Examinadora abaixo.

\section{Prof. Carlos Viana de Carvalho}

Orientador

Departamento de Economia - Pontifícia Universidade Católica do Rio de Janeiro (PUC-Rio)

Prof. Waldyr Areosa

Departamento de Economia - PUC-Rio

Prof. André Minella

Departamento de Estudos e Pesquisas - Banco Central do Brasil 
Todos os direitos reservados. É proibida a reprodução total ou parcial do trabalho sem autorização da universidade, do autor e do orientador.

Bruna Mascotte Oliveira de Menezes

Graduada em administração de empresas pelo Instituto Brasileiro de Mercado de Capitais (IBMEC-RJ). Sócia e consultora da Catavento Consultoria.

Ficha Catalográfica

Mascotte Oliveira de Menezes, Bruna

O impacto de choques no preço do petróleo: pequeno modelo para a economia brasileira / Bruna Mascotte Oliveira de Menezes; orientador: Carlos Viana de Carvalho. - Rio de janeiro: Pontifícia Universidade Católica do Rio de Janeiro (PUC-Rio), Departamento de Economia, 2020.

v., 88 f: il. color. ; $30 \mathrm{~cm}$

Dissertação (mestrado) - Pontifícia Universidade Católica do Rio de Janeiro, Departamento de Economia.

Inclui bibliografia

1. Macroeconomia - Teses. 2. Petróleo. 3. Energia. 4. Política monetária. 5. Modelo macroeconômico. 6. Brasil. I. Viana de Carvalho, Carlos. II. Pontifícia Universidade Católica do Rio de Janeiro. Departamento de Economia. III. Título. 
Ao meu avô, Alberto Mascotte, e à Alice Rosa: na certeza de que seguimos juntos sempre, no amor e na fé. 


\section{Agradecimentos}

Agradeço ao meu orientador, Prof. Carlos Viana de Carvalho, pela inspiração ao longo das aulas de Política Monetária e Fiscal, e por todo o suporte durante a elaboração da tese.

A Lucas Santoro, meu parceiro de vida, pela paciência, apoio e companheirismo de sempre. À Titta, por ter alegrado meus momentos de tensão.

Aos meus pais, Thereza e Alvaro, que durante toda a vida se esforçaram para me dar o melhor que podiam, meu amor incondicional. Aos meus irmãos, Victor e Tony, por me forçarem a tentar ser uma pessoa melhor. À minha avó, tios, tias e primos, pela alegria de compartilhar a vida com vocês.

À minha maior sorte, Carol, Antônia e Laura, minhas companheiras desde sempre, e pra sempre. Aos meus amigos queridos: Guilherme, Thaís, Jade e Victor.

A Rui Terra Neto, pelas longas horas dedicadas a me ajudar com a derivação e estimação.

À Clarissa Lins e Guilherme Ferreira, meus sócios na Catavento Consultoria, pelo incentivo e suporte, em todos os sentidos, ao longo dessa trajetória.

Aos meus colegas de mestrado na PUC-Rio, que ajudaram a tornar mais leves os últimos dois anos e meio de muita dedicação, estudo e aprendizado.

A Deus, que me dá todo dia saúde e força vital para ir atrás de meus sonhos e objetivos. 


\section{Resumo}

Mascotte Oliveira de Menezes, Bruna; Viana de Carvalho, Carlos. O impacto de choques no preço do petróleo: pequeno modelo para a economia brasileira. Rio de Janeiro, 2020. 88p. Dissertação de Mestrado - Departamento de Economia, Pontifícia Universidade Católica do Rio de Janeiro.

Mudanças no preço de petróleo são fonte importante para flutuações econômicas, assim como uma proxy para choques globais, por afetar diversas economias simultaneamente. O modelo semi estrutural estimado visa representar uma pequena economia aberta com câmbio flutuante e regime de metas para a inflação, e foi calibrado e estimado com base em dados da economia brasileira. A inovação do modelo traduz-se pela existência de bens energéticos, aqui representados por derivados de petróleo. Estes têm seus preços influenciados pela cotação do petróleo internacional e são utilizados como insumo para a produção do bem final não energético, além de entrarem diretamente na cesta de consumo das famílias: refletindo o uso de gasolina para deslocamento, profissional e de lazer, e o uso de GLP para cozimento de alimentos. Neste modelo, um choque de $6 \%$ na cotação internacional do petróleo leva a um aumento de $0,6 \%$ na inflação headline e de cerca de $0,4 \%$ na inflação core logo após o choque, que rapidamente retornam ao estado estacionário. A resposta da política monetária varia a depender se o Banco Central responde a desvios na inflação headline ou na inflação core, sendo o aumento dos juros menor para o segundo cenário. Ainda, uma regra de política monetária forward-looking tende a incorrer em maiores aumentos na taxa de juros por menos tempo, sendo menos contracionista. A resposta contracionista gera uma queda de $0,12 \%$ a $0,18 \%$ no consumo trimestral das famílias de forma hump-shaped, notadamente diante do menor consumo de bens energéticos (queda de $2 \%$ ), mas também de bens não energéticos (queda de $0,06 \%$ a $0,12 \%)$.

\section{Palavras-chave}

Petróleo Energia Política monetária Modelo macroeconômico Brasil 


\section{Abstract}

Mascotte Oliveira de Menezes, Bruna; Viana de Carvalho, Carlos (Advisor). The impact of oil price shocks: a small model for the Brazilian economy. Rio de Janeiro, 2020. 88p. Dissertação de Mestrado - Departamento de Economia, Pontifícia Universidade Católica do Rio de Janeiro.

Changes in the oil price are a source of relevant economic fluctuations, as well as a proxy for global shocks, since they affect different economies simultaneously. The estimated semi structural model aims to represent a small open economy with floating exchange rate and inflation targeting, having been calibrated and estimated based on Brazilian data. The innovation in this model lies on the inclusion of the energy sector, hereby represented by oil products. Their prices are influenced by the international oil price and they are used both for the production of the non-energy good, and in the household consumption basket, in order to represent the use of gas for commuting and leisure, as well as the use of liquefied petroleum gas for cooking. In this model, a $6 \%$ increase in the oil price leads to a $0.6 \%$ increase in headline inflation and a $0.4 \%$ increase in core inflation right after the shock, rapidly returning to its steady state level. The Central Bank reaction depends on whether the monetary policy rule responds to deviations from headline or core inflation. The interest rate increase is lower in the second scenario. Also, a forward-looking monetary policy rule leads to a higher increase in the nominal interest rate, but for a shorter period, being less contractionary, since it foresees that the oil price shock will have a short-lived impact on inflation. This contractionary policy leads to a hump-shaped $0.12-0.18 \%$ decrease in quarterly household consumption, notably due to a reduction in demand for energy goods ( $2 \%$ fall), but also for non-energy goods $(0.06-0.12 \%$ fall).

\section{Keywords}

Oil Energy Monetary policy Macroeconomic model Brazil 


\section{Sumário}

1 Introdução 10

2 Modelo $\quad 12$

2.1 Famílias 12

$\begin{array}{lll}2.2 & \text { Firmas } & 14\end{array}$

2.3 Política monetária 17

3 Dados e estimação $\quad 19$

$\begin{array}{lll}3.1 \text { Dados } & 19\end{array}$

3.2 Estimação 21

$\begin{array}{lll}3.3 & \text { Parâmetros calibrados } & 21\end{array}$

3.4 Parâmetros estimados - priors 22

4 Resultados $\quad 24$

4.1 Choque de política monetária 26

4.2 Choque nos preços internacionais do petróleo 29

4.3 Choque nos preços nacionais de derivados de petróleo 31

5 Conclusão 33

6 Referências bibliográficas $\quad 34$

7 Apêndice $\quad 36$

7.1 Equações log linearizadas 36

7.2 Derivação da cesta de consumo das famílias ótima e seu preço 37

7.3 Derivação e linearização da cesta de insumos das firmas produtoras de bens finais (simétrica ao consumo) 39

7.4 Diagnósticos e gráficos para Alternativa Alternativa I: Forward-looking e headline inflation $\quad 40$

7.5 Resultados da estimação de diferentes regras de política monetária 46

7.6 Teste de robustez: série de dados começando em 2005

7.7 Versão com calibração de parâmetro não identificado 80 
"The bloom of health, and life, to man will bring; As from her depths the magic liquid flows, To calm our sufferings, and assuage our woes."

Daniel Yergin, The Prize: The Epic Quest for Oil, Money, and Power. 


\section{1 \\ Introdução}

Desde os anos 70, macroeconomistas vêem mudanças no preço de petróleo como fonte importante para flutuações econômicas, assim como uma proxy para choques globais, por afetar diversas economias simultaneamente. Tal percepção estaria influenciada pelos dois episódios históricos de choques de petróleo acompanhados de baixo crescimento econômico, alto desemprego e alta inflação na década de 70, durante a Guerra de Yom Kippur em 1973 e a Revolução Iraniana em 1979 (Blanchard e Gali, 2007).

A literatura econômica que modela o efeito de choques de petróleo sobre a economia e seus canais de transmissão cresceu, portanto, a partir das grandes variações do preço de petróleo na década de 70. Um primeiro grupo de papers buscou avaliar de forma empírica o tamanho da elasticidade preço do petróleo/PIB (Rasche e Tatom, 1981). Bodenstein et al (Bodenstein et al, 2010), porém, afirmam que tais estudos eram afetados por questões relativas à endogeneidade. Trabalhos subsequentes buscaram endereçar tal questão, ao separar variações do preço do petróleo de episódios onde todos os preços variavam, decompondo, por meio de VARs, o efeito direto dos choques de petróleo e da resposta da política monetária sobre o produto e outras variáveis (Hamilton, 1983). Porém, o uso de VARs com interpretação estrutural na forma reduzida dos coeficientes poderia dificultar a identificação da contribuição da política monetária e a avaliação de regimes alternativos da mesma (Medina e Soto, 2005).

Neste cenário, este estudo busca utilizar um pequeno modelo em parte microfundamentado visto que este tipo de modelo, além de ter se consolidado como importante insumo para tomada de decisão de bancos centrais ao redor do mundo, desde as publicações de Cristiano et al (Cristiano et al, 2005) e Smets e Wouters (Smets e Wouters, 2003), também apresenta vantagens para a estimação do impacto de choques no preço do petróleo na economia. Notadamente, seu uso permite o controle para a causalidade reversa (Bodenstein et al, 2010) e um melhor entendimento dos canais de transmissão do choque no preço do petróleo sobre a inflação e o produto, além da resposta endógena da autoridade monetária (Medina e Soto, 2005). Ainda, papers mais recentes buscam avaliar diferentes regras de política monetária e suas respostas a choques no preço do petróleo, notadamente no que diz respeito à escolha de 
estabilizar a inflação headline ou a inflação core (Medina e Soto, 2005).

Como base para parametrização do pequeno modelo proposto usou-se notadamente o SAMBA, Stochastic Analytical Model with a Bayesian Approach (Castro et al, 2011). De modo a incorporar choques no preço do petróleo, usou-se como base Bodenstein et al (Bodenstein et al, 2008) e Medina e Soto (Medina e Soto, 2005). Os bens energéticos, aqui representados pelos derivados de petróleo, têm seus preços influenciados pela cotação do petróleo internacional e são utilizados como insumo para a produção do bem final não energético, além de entrarem diretamente na cesta de consumo das famílias: refletindo o uso de gasolina para deslocamento, profissional e de lazer, e o uso de GLP para cozimento de alimentos.

Neste sentido, um choque de $6 \%$ na cotação internacional do petróleo leva a um aumento de $0,6 \%$ na inflação headline e de cerca de $0,4 \%$ na inflação core logo após o choque, que rapidamente retornam ao estado estacionário.A resposta da política monetária varia a depender se o Banco Central responde a desvios na inflação headline ou na inflação core, sendo o aumento dos juros menor para o segundo cenário. Ainda, uma regra de política monetária forward-looking tende a incorrer em maiores aumentos na taxa de juros por menos tempo, sendo menos contracionista, por enxergar que o choque de inflação é de curta duração. A resposta contracionista gera uma queda de $0,12 \%$ a $0,18 \%$ no consumo trimestral das famílias de forma hump-shaped, notadamente diante do menor consumo de bens energéticos (queda de 2\%), mas também de bens não energéticos (queda de 0,06\% a 0,12\%).

Este trabalho está organizado da seguinte forma: na seção 2 é apresentado o modelo; a seção 3 descreve os dados utilizados e a estimação realizada; a seção 4 apresenta os resultados da estimação e dos choques no modelo; a seção 5 conclui. 


\section{2}

\section{Modelo}

O modelo semi estrutural estimado visa representar uma pequena economia aberta com câmbio flutuante e regime de metas para a inflação. A inovação do modelo traduz-se pela existência de bens energéticos, derivados de petróleo, na cesta de consumo das famílias e nos insumos das firmas para produção do bem final não energético. O modelo inclui friç̧ões do tipo persistência de hábitos nas preferências do consumidor e rigidez de preços, com fixação de preços à la Calvo.

Um diagrama resumido encontra-se abaixo:

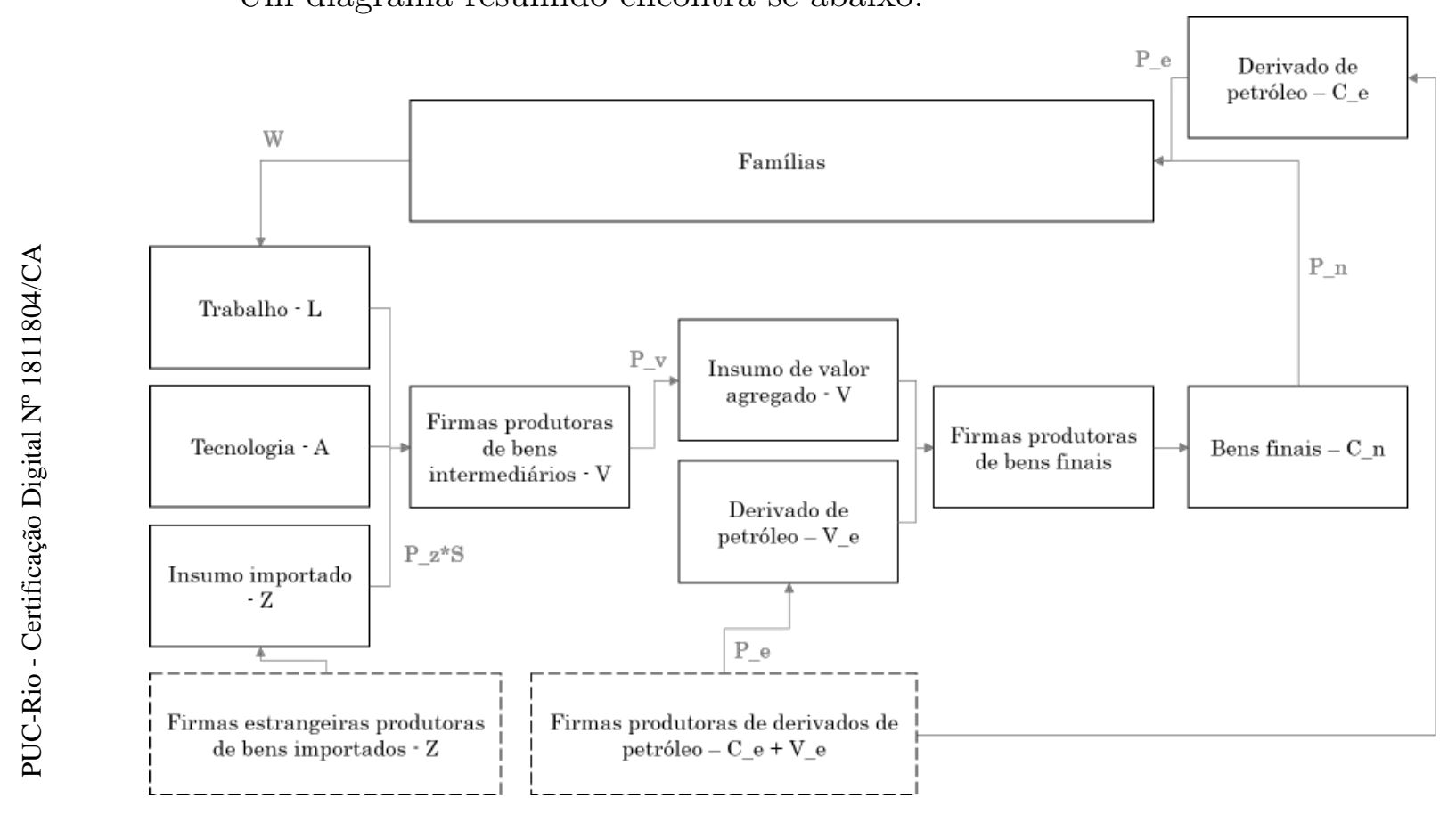

\section{1}

\section{Famílias}

O consumidor representativo maximiza

$$
E_{0} \sum_{t=0}^{\infty} \beta^{t} \Gamma\left(\frac{\left(C_{t}-h \bar{C}_{t-1}\right)^{1-\sigma}-1}{1-\sigma}-\frac{L_{t}^{1+\varphi}}{1+\varphi}\right),
$$

onde $\beta_{t}$ é o fator de desconto intertemporal, $\sigma$ e $\varphi$ são os inversos das elasticidades intertemporais de substituição e da oferta de trabalho, respectivamente. $L_{t}$ é a oferta de trabalho e $h$ representa a persistência dos hábitos de consumo. $\bar{C}_{t-1}$ é o consumo agregado no trimestre anterior, tomado como exógeno pelo agente representativo, sujeito à restrição orçamentária: 


$$
P_{t} C_{t}+E_{t}\left[Q_{t, t+1} B_{t+1}\right]=B_{t}+W_{t} L_{t}+\int \Pi_{t}(i) d i
$$

onde

$$
C_{t}=\left[\left(1-w_{e, t}\right)^{\frac{1}{\rho_{e}}} C_{n, t}^{\frac{\rho_{e}-1}{\rho_{e}}}+\left(w_{e, t}\right)^{\frac{1}{\rho_{e}}} C_{e, t}^{\frac{\rho_{e}-1}{\rho_{e}}}\right]^{\frac{\rho_{e}}{\rho_{e}-1}}
$$

sendo $C_{n_{t}}$ o consumo de bens não energéticos, $C_{e_{t}}$ o consumo de derivados de petróleo, $\rho_{e}$ a elasticidade de substituição entre consumo de bens energéticos e consumo de bens não energéticos e $w_{e, t}$ a importância de bens derivados de petróleo na cesta de consumo. Este último pode variar ao longo do tempo, e seu log em torno do estado estacionário $\left(\hat{w}_{e, t}\right)$ segue um processo $\operatorname{AR}(1)$ do tipo:

$$
\hat{w}_{e, t}=\rho_{w_{e, t}} \hat{w}_{e, t}+\sigma_{w_{e, t}} \varepsilon_{t}^{w_{e}},
$$

onde $\varepsilon_{t}^{w_{e}}$ são choques no peso de bens derivados de petróleo na cesta de consumo.

O preço real dos derivados de petróleo nacionais é calculado a partir da divisão do nível de preços:

$$
P_{e_{t}}^{r}=\frac{P_{e_{t}}}{P_{t}}
$$

O movimento dos preços reais dos derivados de petróleo é dado por

$$
P_{e_{t}}^{r}=P_{e_{t}}^{*_{r}} S_{t}^{r} \epsilon_{t}^{P_{e}}
$$

onde $P_{e_{t}}^{*_{r}}$ é a cotação internacional dos preços reais de petróleo, $S_{t}$ é a taxa de câmbio e $\epsilon_{t}^{P_{e}}$ são choques nos preços reais internos de derivados de petróleo. Tais choques cumprem o papel de representar os processos (ex.: refino do óleo cru em derivado de petróleo) ou particularidades brasileiras (ex.: controle artificial de preços administrados e mudanças na política de preços da Petrobras) que fazem com que o preço do derivado nacional não seja igual ao preço do petróleo internacional convertido pela taxa de câmbio vigente, e seguem

$$
\epsilon_{t}^{P_{e}}=\rho_{\epsilon}{\mathcal{P}_{e}}_{t-1}^{P_{e}}+\sigma_{\epsilon_{P_{e}}} \varepsilon_{t}^{P_{e}}
$$

Já os movimentos na cotação internacional do preço real do petróleo, em desvio log do estado estacionário $\left(p_{e_{t}}^{*_{r}}\right)$, seguem

$$
p_{e_{t}}^{*_{r}}=\rho_{p_{e}^{*}} p_{e_{t-1}}^{*_{r}}+\sigma_{p_{e}^{*}} \varepsilon_{t}^{p_{e}^{*}}
$$


onde $\varepsilon_{t}^{P_{e}^{*}}$ são choques nos preços reais internacionais de petróleo.

A partir de $G_{t}=\frac{\Gamma_{t+1}}{\Gamma_{t}}$, supomos que, em desvios logarítmicos de seu steady state, o processo $G_{t}$ satisfaça

$$
g_{t}=\rho_{g} g_{t-1}+\sigma_{g} \varepsilon_{t}^{g}
$$

Por meio das condições de primeira ordem, encontramos as seguintes equações para a demanda por derivados de petróleo $\left(C_{e_{t}}\right)$ e bens finais $\left(C_{n_{t}}\right)$ por parte das famílias

$$
\begin{gathered}
C_{n, t}=\left(\frac{P_{n, t}}{P_{t}}\right)^{-\rho_{e}}\left(1-w_{e, t}\right) C_{t}, \\
C_{e, t}=\left(\frac{P_{e, t}}{P_{t}}\right)^{-\rho_{e}}\left(w_{e, t}\right) C_{t},
\end{gathered}
$$

e o preço da cesta de consumo das famílias é dado por

$$
P_{t}^{1-\rho_{e}}=\left(1-w_{e, t}\right) P_{n, t}^{1-\rho_{e}}+w_{e, t} P_{e, t}^{1-\rho_{e}} .
$$

\section{2}

\section{Firmas}

O bem final não energético consumido pelas famílias, $C_{n_{t}}$, é produzido pelas firmas produtoras de bens finais, que operam sob competição perfeita, por meio das funções de produção e custo abaixo

$$
\begin{gathered}
Y_{t}=\left[\left(1-w_{e_{y}, t}\right)^{\frac{1}{\rho_{e}}} V_{t}^{\frac{\rho_{e_{y}}-1}{\rho_{e_{y}}}}+\left(w_{e_{y}, t}\right)^{\frac{1}{\rho_{e}}} V_{e, t}^{\frac{\rho_{e}-1}{\rho_{e}}}\right]^{\frac{\rho_{e_{y}}}{\rho_{e_{y}}-1}}, \\
\text { Custo }{ }_{Y}=V_{t} P_{v_{t}}+V_{e_{t}} P_{e_{t}},
\end{gathered}
$$

onde $V_{t}$ é o insumo de valor agregado produzido pelas firmas produtoras de bens intermediários, $V_{e_{t}}$ é a demanda de derivados de petróleo para produção do produto final, $\rho_{e_{y}}$ é elasticidade de substituição entre o derivado de petróleo e insumo de valor agregado e $w_{e_{y}, t}$ é a relevância do petróleo na produção do produto final. Este último pode variar ao longo do tempo, e seu log em torno do estado estacionário $\left(\hat{w}_{e_{y}, t}\right)$ segue um processo $\operatorname{AR}(1)$ do tipo:

$$
\hat{w}_{e_{y}, t}=\rho_{w_{e y}} \hat{w}_{e_{y}, t-1}+\sigma_{w_{e_{y}}} \varepsilon_{t}^{w_{e_{y}}}
$$

onde $\varepsilon_{t}^{w_{e}}$ são choques no peso de bens derivados de petróleo na cesta de insumos para produção do bem final.

A partir das condições de primeira ordem, temos as seguintes equações de demanda por insumo derivado de petróleo $\left(v_{e, t}\right)$, insumo de valor agregado $\left(v_{t}\right)$ e preço do bem final $\left(P_{n_{t}}\right)$ : 


$$
\begin{gathered}
V_{t}=\left(\frac{P_{v, t}}{P_{n, t}}\right)^{-\rho_{e_{y}}}\left(1-w_{e_{y}, t}\right) Y_{t} \\
V_{e, t}=\left(\frac{P_{e, t}}{P_{n, t}}\right)^{-\rho_{e_{y}}}\left(w_{e_{y}, t}\right) Y_{t} \\
P_{n_{t}}^{1-\rho_{e_{y}}}=\left(1-w_{e_{y}, t}\right) P_{v, t}^{1-\rho_{e_{y}}}+w_{e_{y}, t} P_{e, t}^{1-\rho_{e_{y}}}
\end{gathered}
$$

O composto das diferentes variedades de insumos de valor agregado, $V_{t}$, e o preço do bem de valor agregado, $P_{V_{t}}$ se dão por

$$
\begin{aligned}
V_{t} & =\left(\int_{0}^{1} V_{t}(i)^{\frac{\theta_{t-1}}{\theta_{t}}} d i\right)^{\frac{\theta_{t}}{\theta_{t-1}}}, \\
P_{V_{t}} & =\left(\int_{0}^{1} P_{V_{t}}(i)^{1-\theta_{t}} d i\right)^{\frac{1}{1-\theta_{t}}},
\end{aligned}
$$

onde $V_{t}(i)$ são as diferentes variedades de insumos de valor agregado e $P_{v_{t}}(i)$ são os preços das diferentes variedades de insumos de valor agregado. Tais insumos são produzidos por firmas produtoras de bens intermediários que serão introduzidas mais adiante, e a demanda por cada variedade resultante da otimização é dada por

$$
V_{t}(i)=V_{t} *\left(\frac{P_{v_{t}}(i)}{P_{v_{t}}}\right)^{-\theta_{t}}
$$

Ainda, o equilíbrio no mercado de bens finais é dado, portanto, por

$$
Y_{t}=C_{n, t}
$$

ou seja, a produção de bens finais deve ser igual à demanda das famílias pelos bens finais.

Os insumos de valor agregado $\left(V_{t}\right)$ são produzidos por um continuum de firmas que operam sob competição monopolística, de acordo com as funções de produção e custo abaixo

$$
\begin{aligned}
V_{t}(i) & =A L_{t}(i)^{(1-\delta)} Z_{t}(i)^{\delta}, \\
\text { Custo }_{V} & =W_{t} L_{t}(i)+S_{t} P_{t}^{*} Z_{t}(i),
\end{aligned}
$$

onde $A$ é a tecnologia, $L_{t}(i)$ é a demanda por trabalho, $Z_{t}(i)$ é a quantidade de insumo importado utilizado pela firma i e $V_{t}(\mathrm{i})$ é o insumo de valor agregado produzido pela firma i.

Em desvio log do steady state, o processo de produtividade $A_{t}$ satisfaz

$$
a_{t}=\rho_{a} a_{t-1}+\sigma_{a} \varepsilon_{t}^{a}
$$

O preço do insumo importado para a produção de bens intermediários, $P_{t}^{*}$, é determinado no mercado internacional e satisfaz (em desvios log do steady state): 


$$
\pi_{t}^{*}=\rho_{\pi}^{*} \pi_{t-1}^{*}+\sigma_{\pi}^{*} \varepsilon_{t}^{\pi^{*}}
$$

onde

$$
\pi_{t}^{*}=\ln \left(\frac{P_{t}^{*}}{P_{t-1}^{*}}\right)-\bar{\pi}
$$

e $\bar{\pi}$ é a inflação de steady state.

Já a taxa de câmbio $S_{t}$, em desvios log do steady state, satisfaz:

$$
i_{t}=i_{t}^{*}+E_{t}\left[s_{t+1}\right]-s_{t}+\phi_{t}-\chi\left(s_{t}+p_{t}^{*}-p_{t}\right)
$$

onde $i_{t}^{*}$ é a taxa de juros dos EUA (em desvios log do steady state) e satisfaz

$$
i_{t}^{*}=\rho_{i^{*}} i_{t-1}^{*}+\sigma_{i}^{*} \varepsilon_{t}^{i^{*}}
$$

$i_{t}$ é a taxa de juros fixada pelo Banco Central de acordo com a regra de juros a ser definida posteriormente, e $\phi_{t}$ é um choque de prêmio de risco que satisfaz

$$
\phi_{t}=\rho_{\phi} \phi_{t-1}+\sigma_{\phi} \varepsilon_{t}^{\phi}
$$

$\mathrm{Na}$ presença de Loterias de Calvo, as firmas intermediárias produtoras de bens de valor agregado minimizam seus custos por meio de

$$
\begin{gathered}
\max _{L_{t}, Z_{t}} W_{t} L_{t}(i)+S_{t} P_{t}^{*} Z_{t}(i) \\
\text { s.a. } A_{t} L_{t}(i)^{(1-\delta)} Z_{t}(i)^{\delta}
\end{gathered}
$$

Além de escolher $L_{t}$ e $Z_{t}$ que minimizem seu custo, quando a firma i é sorteada a reotimizar seus preços, ela maximizará seu valor presente ponderado pela probabilidade de não reotimização dada por $(1-\lambda)$ e a sequência de demanda que ela enfrentará:

$$
\begin{gathered}
\max _{P_{V_{t}}^{0}} \sum_{k=0}^{\infty}(1-\lambda)^{k} E_{t} Q_{t, t+k}\left[P_{V_{t}}^{0} \Pi_{t-1, t+k-1}^{\gamma} V_{t+k \mid t}(i)-V_{t}(i)\right] \\
\text { s.a. } V_{t+k \mid t}(i)=\left(\frac{P_{V_{t}}^{0}}{P_{V_{t+k}}}\right)^{-\theta_{t+k}} \prod_{t-1, t+k-1}^{-\gamma \theta_{t+k}} V_{t+k}
\end{gathered}
$$

onde $P_{V_{t}}^{0}$ é o preço do insumo de valor agregado que a firma escolhe caso seja sorteada. Quando não reotimiza, seus preços são indexados, sendo corrigidos por uma fração da inflação passada:

$$
P_{V_{t}}(i)=P_{V_{t-1}} *\left(\frac{P_{V t-1}}{P_{V t-2}}\right)^{\gamma}
$$

Ainda, 


$$
M_{t}=\frac{\theta_{t}}{\theta_{t}-1},
$$

onde $\theta_{t}$ é a elasticidade de substituição entre as variedades de insumos de valor agregado e $M_{t}$ é o mark-up dos preços cobrados pelas firmas produtoras de bens intermediários que evolui, em desvios logarítmos do estado estacionário, de acordo com

$$
\hat{M}_{t}=\rho_{M} \hat{M}_{t-1}+\sigma_{M} \varepsilon_{t}^{M}
$$

\section{3}

\section{Política monetária}

\subsection{1}

\section{Alternativa I: Forward-looking e headline inflation}

O Banco Central fixa a taxa de juros doméstica trimestral de acordo com a seguinte regra de Taylor (em desvios log do steady state):

$i_{t}=\rho_{i} i_{t-1}+\left(1-\rho_{i}\right)\left[\phi_{\pi}\left(\frac{E_{t}\left[\pi_{t-1, t+2}\right]-\pi^{m}}{4}\right)+\phi_{c} c_{t}+\phi_{\Delta_{c}}\left(c_{t}-c_{t-4}\right)+\phi_{s}\left(s_{t}-s_{t-2}\right)\right]+v_{t}$

onde $\pi^{m}$ a é meta para a inflação anual (em desvio log do steady state), $\pi_{t-1, t+2}$ é a inflação acumulada nos trimestres entre $\mathrm{t}-1$ e $\mathrm{t}+2$ e $v_{t}$ é um choque monetário, tal que:

$$
\begin{gathered}
\pi_{t}^{m}=\rho_{\pi^{m}} \pi_{t-1}^{m}+\sigma_{\pi^{m}} \varepsilon_{t}^{\pi^{m}} \\
v_{t}=\rho_{v} v_{t-1}+\sigma_{v} \varepsilon_{t}^{v}
\end{gathered}
$$

\subsection{2}

\section{Alternativa II: Forward-looking e core inflation}

O Banco Central fixa a taxa de juros doméstica de acordo com a seguinte regra de Taylor (em desvios log do steady state):

$i_{t}=\rho_{i} i_{t-1}+\left(1-\rho_{i}\right)\left[\phi_{\pi}\left(\frac{\left(E_{t}\left[\pi_{n_{t-1, t+2}}\right]-\pi^{m}\right.}{4}\right)+\phi_{c} c_{t}+\phi_{\Delta_{c}}\left(c_{t}-c_{t-4}\right)+\phi_{s}\left(s_{t}-s_{t-2}\right)\right]+v_{t}$

onde $\pi^{m}$ a é meta para a inflação anual (em desvio log do steady state):

$$
\pi_{t}^{m}=\rho_{\pi^{m}} \pi_{t-1}^{m}+\sigma_{\pi^{m} \varepsilon_{t}^{\pi^{m}}}
$$

$\pi_{n_{t-1, t+2}}$ é a inflação core acumulada nos trimestres entre $\mathrm{t}-1$ e $\mathrm{t}+2 \mathrm{a}$ partir de

$$
\pi_{n_{t}}=\ln \left(\frac{P_{N_{t}}}{P_{N_{t}-1}}\right)-\bar{\pi}_{n}
$$

e $\bar{\pi}_{n}$ é a inflação core de steady state

e $v_{t}$ é um choque monetário:

$$
v_{t}=\rho_{v} v_{t-1}+\sigma_{v} \varepsilon_{t}^{v}
$$




\subsection{3}

\section{Alternativa III: Backward-looking e headline inflation}

O Banco Central fixa a taxa de juros doméstica de acordo com a seguinte regra de Taylor (em desvios log do steady state):

$i_{t}=\rho_{i} i_{t-1}+\left(1-\rho_{i}\right)\left[\phi_{\pi}\left(\frac{\pi_{t-3, t}-\pi^{m}}{4}\right)+\phi_{c} c_{t}+\phi_{\Delta_{c}}\left(c_{t}-c_{t-4}\right)+\phi_{s}\left(s_{t}-s_{t-2}\right)\right]+v_{t}$

onde $\pi^{m}$ a é meta para a inflação anual (em desvio log do steady state), $\pi_{t-3, t}$ é a inflação acumulada nos últimos trimestres entre t - 3 e t e $v_{t}$ é um choque monetário, tal que:

$$
\begin{gathered}
\pi_{t}^{m}=\rho_{\pi^{m}} \pi_{t-1}^{m}+\sigma_{\pi^{m}} \varepsilon_{t}^{\pi^{m}} \\
v_{t}=\rho_{v} v_{t-1}+\sigma_{v} \varepsilon_{t}^{v}
\end{gathered}
$$

\subsection{4}

\section{Alternativa IV: Backward-looking e core inflation}

O Banco Central fixa a taxa de juros doméstica de acordo com a seguinte regra de Taylor (em desvios log do steady state):

$i_{t}=\rho_{i} i_{t-1}+\left(1-\rho_{i}\right)\left[\phi_{\pi}\left(\frac{\pi_{n_{t-3, t}-\pi^{m}}}{4}\right)+\phi_{c} c_{t}+\phi_{\Delta_{c}}\left(c_{t}-c_{t-4}\right)+\phi_{s}\left(s_{t}-s_{t-2}\right)\right]+v_{t}$ onde $\pi^{m}$ a é meta para a inflação anual (em desvio log do steady state)

$$
\pi_{t}^{m}=\rho_{\pi^{m}} \pi_{t-1}^{m}+\sigma_{\pi^{m}} \varepsilon_{t}^{\pi^{m}}
$$

$\pi_{n_{t-3, t}}$ é a inflação acumulada nos últimos trimestres entre t - 3 e t

$$
\pi_{n_{t}}=\ln \left(\frac{P_{N_{t}}}{P_{N_{t}-1}}\right)-\bar{\pi}_{n}
$$

e $\bar{\pi}_{n}$ é a inflação core de steady state

e $v_{t}$ é um choque monetário:

$$
v_{t}=\rho_{v} v_{t-1}+\sigma_{v} \varepsilon_{t}^{v}
$$

Como na maior parte da literatura sobre modelos DSGE, as equações de equilíbrio foram submetidas à aproximação de primeira ordem em torno de um estado estacionário. O conjunto completo de equações log-linearizadas se encontra no Apêndice. 


\section{3}

\section{Dados e estimação}

\section{1}

\section{Dados}

A estimação usou como base dados trimestrais de janeiro de 2000 a dezembro de 2019, de modo a englobar o período de vigência do regime de metas de inflação no Brasil. Para a inflação, utilizamos a variação trimestral do Índice de Preços ao Consumidor Amplo, computado pelo IBGE/SNIPC e disponível no ipeadata. Para a taxa de juros doméstica, a média trimestral da taxa Selic, também disponível no ipeadata, foi trimestralizada com base na fórmula:

$$
\text { média_Selic_trim }=\left((1+\text { média_Selic_anual })^{1 / 4}\right)-1 \text {. }
$$

Para o consumo, foi utilizado o dado de consumo das famílias no Sistema de Contas Nacionais (SCN) do IBGE. A taxa de câmbio BRL/USD utilizada é a média trimestral do dado disponibilizado no ipeadata pelo Banco Central/SGS. A inflação estrangeira é calculada por meio da variação trimestral do Consumer Price Index (CPI) dos EUA, disponível no website do Federal Reserve Bank de St. Louis (FRED).

Para a taxa de juros estrangeira, tendo em vista que o período de análise perpassa o período de zero lower bound, foi utilizada a Wu-Xia Shadow Federal Funds Rate (Wu e Xia, 2016) trimestralizada pela mesma fórmula utilizada para a Selic, de modo a incorporar o impacto das medidas de política monetária não convencionais, como quantitative easing, em uma taxa de juros teórica.

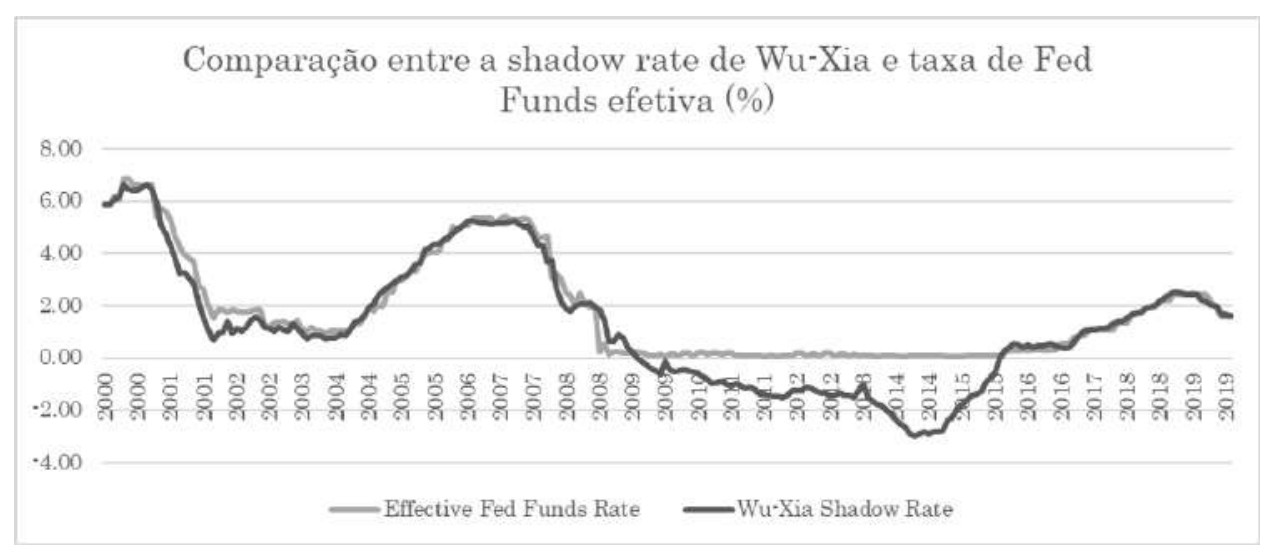

Para os preços internacionais de petróleo, usou-se a cotação média trimestral dos preços de petróleo do tipo Brent, considerado benchmark inter- 
nacional para a commodity, e disponível no site do Federal Reserve Bank de St. Louis (FRED).

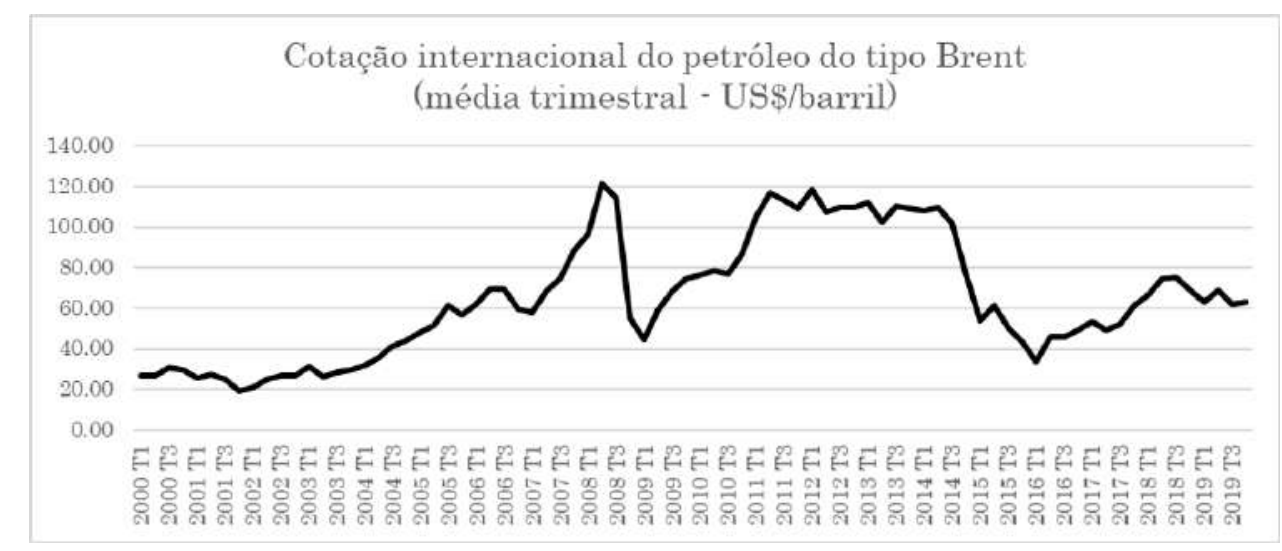

Para os preços de derivados nacionais, construiu-se um índice ponderado (base 2018) com a variação de preços da gasolina, do óleo diesel e do GLP, que juntos respondem por cerca de $90 \%$ do mercado de derivados de petróleo brasileiro (Agência Nacional de Petróleo, Gás Natural e Biocombustíveis/ANP, 2019) como pode ser observado nos gráficos abaixo:

Consumo de derivados de petróleo no Brasil (2018)

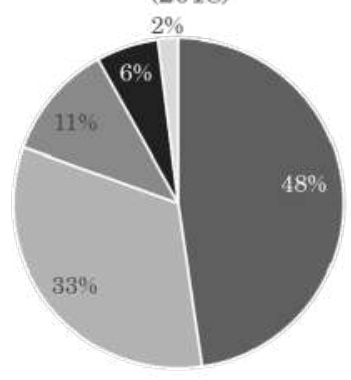

- Óleo diesel

- Gasolina comum

-GLP

- QAV

Consumo de derivados de petróleo no Brasil (média 2009-2018)

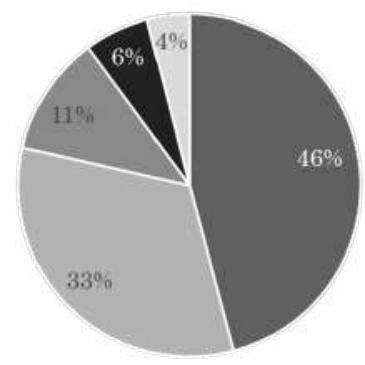

Fonte: Agéncia Nacional do Petróleo, Gás Natural e Biocombustíveis - ANP

Por fim, para a demanda por derivados de petróleo das famílias usou-se como proxy o consumo aparente de gasolina e GLP, disponível no ipeadata. Para a demanda por derivados das firmas, usou-se como proxy o consumo aparente de óleo diesel, combustível usado para transporte de bens e mercadorias via modal rodoviário, responsável por 91\% da demanda energética para transporte de cargas no Brasil em 2019 (Empresa de Pesquisa Eenergética/MME, 2019).

Todos os dados, em percentual ou em log do nível, foram submetidos ao teste ADF para raiz unitária. A estatística ADF, usada no teste, é um número 
negativo, e quanto mais negativo, mais indicativo o teste se torna de rejeitar a hipótese nula de que existe raiz unitária na série. Os resultados dos testes ADF nas séries primárias são apresentados abaixo.

\begin{tabular}{|l|r|r|l|l|}
\hline Resultado testes ADF & t-stat & critical value 5\% & raiz unitária? & tratamento \\
\hline log consumo das famílias (R\$) & -2.10 & -2.8922 & sim & ciclo - filtro HP \\
\hline log consumo de derivados de petróleo pelas famílias (1) & -0.67 & -2.8922 & sim & ciclo - filtro HP \\
\hline taxa de juros nominal (\%) & -0.96 & -2.8932 & sim & ciclo- filtro HP \\
\hline taxa de juros nominal estrangeira (\%) & -3.50 & -2.8943 & não & desvio da média amostral \\
\hline meta de inflação (\%) & -6.80 & -2.8922 & não & desvio da média amostral \\
\hline log preço real de derivados de petróleo nacionais (R\$) & -2.55 & -2.8922 & sim & ciclo- filtro HP \\
\hline log preço real de petróleo internacional (US\$) & -1.77 & -2.8922 & sim & ciclo - filtro HP \\
\hline inflação (\%) & -4.93 & -2.8922 & não & desvio da média amostral \\
\hline inflação estrangeira (\%) & -9.21 & -2.8922 & não & desvio da média amostral \\
\hline log taxa de câmbio (US\$/R\$) & -1.48 & -2.8922 & sim & ciclo - filtro HP \\
\hline log consumo de derivados de petróleo pelas firmas (1) & -1.20 & -2.8950 & sim & ciclo- filtro HP \\
\hline
\end{tabular}

Naqueles onde havia raiz unitária, como consumo das famílias, consumo de derivados de petróleo, preço de petróleo e taxa Selic, o tratamento foi baseado em retirar a tendência por meio do filtro HP, com $\lambda=1600$. No caso de séries que não continham raiz unitária, como as taxas de inflação nacional e estrangeira, foi calculado o desvio da média amostral.

\section{2}

\section{Estimação}

Para a estimação do modelo, foi especificado um grupo de parâmetros a serem calibrados, além de priors para os parâmetros estimados. As posteriors foram geradas por meio do algoritmo Metropolis-Hastings, utilizando o método de fases adaptativas. Para cada uma das versões de política monetária, foi feita uma primeira estimação com 10.000 replicações, onde foram descartadas 5.000. A partir de então, foram feitas estimações seguintes com 20.000, 50.000 e 100.000 replicações, descartando metade e utilizando a matriz de covariância da estimação anterior por meio do comando do dynare mcmc_jumping _covariance.

\section{3}

\section{Parâmetros calibrados}

O modelo estimado tem um total de 41 parâmetros. Desse total, calibraram-se 10 e estimaram-se 31. Em relação à calibragem dos parâmetros, utilizou-se como base a estimação de Castro et al. (2011), de Bodenstein et al (2010) e dados do setor energético brasileiro.

O primeiro parâmetro foi $\chi$, calibrado como $1^{e-3}$, bem pequeno, suficiente apenas para garantir a unicidade de equilíbrio estacionário sem afetar a 
dinâmica da economia.

O segundo parâmetro calibrado foi $\beta$, o fator de desconto. Utilizamos o valor de 0.989 , conforme calculado por Castro et al (2011) tomando como base a taxa de crescimento do PIB brasileiro, a taxa de juros média entre 2005Q1-2010Q2 e a meta de inflação então vigente de 4,5\%.

O terceiro parâmetro calibrado foi $\mathrm{h}=0.74$, com base no fator de persistência de hábitos estimado por Castro et al (2011), e em linha com o valor utilizado por Linardi (2016) (Linardi, 2016) de 0.7. O inverso da elasticidade de substituição do trabalho, $\varphi$, foi calibrado em 1, conforme Castro et al (2011).

O peso de derivados de petróleo na cesta de consumo das famílias, $w_{e}$ de 4,267\%, foi calibrado com base no peso da gasolina e do GLP no Índice de Preços ao Consumidor Amplo - IPCA. Já o peso de derivados de petróleo na cesta de insumos das firmas produtoras de bens finais, $w_{e_{y}}$ de $5,547 \%$, foi calibrado com base no peso de derivados de petróleo no Índice de Preços ao Produtor Amplo - IPA-OG, computado pelo FGV-IBRE.

Os demais parâmetros calibrados se encontram na tabela abaixo:

\begin{tabular}{|l|l|r|}
\hline Parâmetros calibrados & símbolo & valor calibrado \\
\hline chi & X & $1.00 \mathrm{E}-03$ \\
\hline fator de desconto & $\beta$ & 0.989 \\
\hline fator de persistência de hábitos & h & 0.74 \\
\hline inverso da elasticidade de substituição do trabalho & varphi & 1 \\
\hline coeficiente da inflação & gamma & 0.5 \\
\hline peso de derivados de petróleo na cesta de consumo & w_e & 0.04267 \\
\hline peso de derivados de petróleo na cesta de insumos para produção do bem final & w_ey & 0.05547 \\
\hline choque monetário & rho_v & 0.3 \\
\hline choque de produtividade & rho_a & 0.8 \\
\hline choque de prêmio de risco & rho_phi & 0.8 \\
\hline
\end{tabular}

\section{4}

\section{Parâmetros estimados - priors}

Para as distribuições das priors, utilizou-se como referência as estimações de Castro et al (2011). As distribuições utilizadas estão resumidas na tabela abaixo: 


\begin{tabular}{|c|c|c|c|}
\hline Priors & distribuição & média & variância \\
\hline$\sigma$ & normal & 1.4 & 0.05 \\
\hline$\delta$ & beta & 0.5 & 0.15 \\
\hline$\rho_{e}$ & beta & 0.4 & 0.15 \\
\hline$\rho_{e_{y}}$ & beta & 0.4 & 0.15 \\
\hline$\rho_{g}$ & beta & 0.5 & 0.25 \\
\hline$\rho_{m u}$ & beta & 0.5 & 0.25 \\
\hline$\rho_{\pi}$ & beta & 0.5 & 0.25 \\
\hline$\rho_{i *}$ & beta & 0.5 & 0.25 \\
\hline$\rho_{i}$ & beta & 0.6 & 0.15 \\
\hline$\rho_{p_{e}^{*}}$ & beta & 0.5 & 0.15 \\
\hline$\rho_{e_{p_{e}}}$ & beta & 0.5 & 0.15 \\
\hline$\rho_{\pi^{m}}$ & beta & 0.6 & 0.15 \\
\hline$\rho_{w_{e}}$ & beta & 0.5 & 0.15 \\
\hline$\rho_{w_{e y}}$ & beta & 0.5 & 0.15 \\
\hline$\phi_{\pi}$ & normal & 2.5 & 0.35 \\
\hline$\phi_{c}$ & gamma & 0.4 & 0.1 \\
\hline$\phi_{d c}$ & gamma & 0.3 & 0.1 \\
\hline$\phi_{s}$ & gamma & 0.7 & 0.1 \\
\hline$\lambda$ & beta & 0.1 & 0.05 \\
\hline$\sigma_{g}$ & inv_gamma & 0.001 & 1 \\
\hline$\sigma_{m u}$ & inv_gamma & 0.1 & 1 \\
\hline$\sigma_{\pi}$ & inv_gamma & 0.001 & 1 \\
\hline$\sigma_{a}$ & inv_gamma & 0.1 & 1 \\
\hline$\sigma_{i^{*}}$ & inv_gamma & 0.001 & 1 \\
\hline$\sigma_{p h i}$ & inv_gamma & 0.001 & 1 \\
\hline$\sigma_{v}$ & inv_gamma & 0.001 & 1 \\
\hline$\sigma_{e_{p_{e}}}$ & inv_gamma & 0.001 & 1 \\
\hline$\sigma_{p_{e}^{*}}$ & inv_gamma & 0.001 & 1 \\
\hline$\sigma_{\pi^{m}}$ & inv_gamma & 0.001 & 1 \\
\hline$\sigma_{w_{e}}$ & inv_gamma & 0.001 & 0.2 \\
\hline$\sigma_{w_{e y}}$ & inv_gamma & 0.001 & 0.2 \\
\hline
\end{tabular}




\section{Resultados}

Os resultados das quatro versões do modelo estimadas se encontram abaixo, onde as diferenças têm como base as alternativas de regra de política monetária. Em geral, as posteriors apresentam padrão normal e as funções de resposta impulso são bem-comportadas, com os sinais e formatos esperados pela teoria econômica.

O valor estimado para a elasticidade de substituição entre o derivado de petróleo e o bem não energético na cesta de consumo das famílias, $\rho_{e}$, é estimado em torno de 0.38, em linha com Bodenstein (2010) e An e Kang (2011) (An e Kang, 2011). Já a elasticidade de substituição entre o derivado de petróleo e o bem não energético na cesta de insumos das firmas produtoras de bens finais, $\rho_{e_{y}}$ é ligeiramente maior, em torno de 0.43 .

A persistência do preço do petróleo internacional, $\rho_{p_{e}^{*}}$, é estimado em torno de 0.70, em linha com Medina e Soto (2005) e An e Kang (2011).

Ainda, no que diz respeito aos parâmetros de política monetária, a persistência da taxa de juros nominal, $\rho_{i}$, foi estimada em cerca de 0.21 , menor do que o resultado de Carvalho e Vilela (2015) (Carvalho e Vilela, 2015), de 0.54. Ainda, o parâmetro de sensibilidade da política monetária à inflação, $\phi_{\pi}$, foi estimado em cerca de 2.04, em linha com Carvalho e Vilela (2005).

Por fim, por meio da observação do gráfico que compara prior x posterior (contido no Apêndice), foi possível observar que o parâmetro $\rho_{w_{e y}}$ não estaria identificado, por apresentar distribuição prior e posterior praticamente idêntica. Ao analisar as possíveis causas de tal fato, acreditamos que tenha ocorrido devido à existência de apenas uma variável observável entre as presentes na cesta de insumos das firmas produtoras de bens finais, sendo ela a demanda por energéticos das firmas $\left(V_{e}\right)$. Já o parâmetro $\rho_{w_{e}}$ foi corretamente identificado, tendo como base as duas variáveis observáveis contidas na cesta de bens das famílias: $C_{e}$ e $C$. Entre as potenciais soluções para tal fato estão a calibração do parâmetro $\rho_{w_{e y}}$ com base no valor estimado para $\rho_{w_{e}}$, o que foi feito sem alterações nos resultados, que podem ser encontrados no Apêndice.

As distribuições posteriores da estimação da primeira alternativa de política monetária (forward-looking e inflação headline), com as respectivas média, intervalo de confiança e desvio padrão, constam na tabela abaixo: 


\begin{tabular}{|c|c|c|c|c|c|c|}
\hline \multirow{2}{*}{$\begin{array}{l}\text { parâmetro } \\
\text { sigma }\end{array}$} & \multirow{2}{*}{\begin{tabular}{|l|} 
prior \\
1.400 \\
\end{tabular}} & \multirow{2}{*}{\begin{tabular}{l|} 
posterior \\
1.4616
\end{tabular}} & \multicolumn{2}{|c|}{$90 \%$ intervalo } & \multirow{2}{*}{\begin{tabular}{|l|} 
distribuição \\
norm
\end{tabular}} & \multirow{2}{*}{\begin{tabular}{|l} 
destio padrão \\
0.0500
\end{tabular}} \\
\hline & & & 1.3798 & 1.5475 & & \\
\hline delta & 0.400 & 0.5409 & 0.3080 & 0.7931 & beta & 0.1500 \\
\hline rho_e & 0.400 & 0.3809 & 0.2159 & 0.5516 & beta & 0.1500 \\
\hline tho_ey & 0.400 & 0.4291 & 0.2536 & 0.6035 & beta & 0.1500 \\
\hline rho_g & 0.500 & 0.0821 & 0.0024 & 0.1535 & beta & 0.2500 \\
\hline tho_mu & 0.500 & 0.2115 & 0.0125 & 0.3788 & beta & 0.2500 \\
\hline rho_pi & 0.500 & 0.1445 & 0.0045 & 0.2767 & beta & 0.2500 \\
\hline rho_i_ext & 0.500 & 0.8327 & 0.7768 & 0.8868 & beta & 0.2500 \\
\hline rho_i & 0.500 & 0.2256 & 0.1023 & 0.3515 & beta & 0.1500 \\
\hline sho_p_e_ext & 0.500 & 0.7042 & 0.5993 & 0.8163 & beta & 0.1500 \\
\hline rho_e.p_e & 0.500 & 0.7134 & 0.6034 & 0.8227 & beta & 0.1500 \\
\hline rho_meta & 0.600 & 0.7453 & 0.6200 & 0.8773 & beta & 0.1500 \\
\hline rho_w_e & 0.500 & 0.4965 & 0.3808 & 0.6167 & beta & 0.1500 \\
\hline rho_w_ey & 0.500 & 0.5006 & 0.2561 & 0.7534 & beta & 0.1500 \\
\hline phi_pi & 2.500 & 2.1547 & 1.6271 & 2.6995 & norm & 0.3500 \\
\hline phi_e & 0.400 & 0.4676 & 0.2758 & 0.6545 & gamm & 0.1000 \\
\hline phi_de & 0.300 & 0.4367 & 0.2185 & 0.6413 & gamm & 0.1000 \\
\hline phi_s & 0.700 & 1.0470 & 0.8764 & 1.2138 & gamm & 0.1000 \\
\hline lambda & 0.100 & 0.0765 & 0.0572 & 0.0963 & beta & 0.0500 \\
\hline sigma_gg & 0.250 & 0.0418 & 0.0353 & 0.0481 & invg & Inf \\
\hline
\end{tabular}

\begin{tabular}{|l|l|l|l|l|l|l|}
\hline parâmetro & prior & posterior & \multicolumn{2}{|l|}{$90 \%$ intervalo } & distribuiçăo & desrio padrão \\
\hline sigma_mu & 0.500 & 0.6268 & 0.3362 & 0.9994 & invg & Inf \\
\hline sigma_pi & 0.025 & 0.0073 & 0.0063 & 0.0084 & invg & Inf \\
\hline sigma_a & 0.500 & 0.1265 & 0.1000 & 0.1553 & invg & Inf \\
\hline sigma_i_ext & 0.025 & 0.0031 & 0.0029 & 0.0033 & invg & Inf \\
\hline sigma_phi & 0.050 & 0.0090 & 0.0076 & 0.0104 & invg & Inf \\
\hline sigma_v & 1.000 & 0.1224 & 0.1176 & 0.1284 & invg & Inf \\
\hline sigma___e_ext & 0.250 & 0.0632 & 0.0544 & 0.0719 & invg & Inf \\
\hline sigma_e_p_e & 0.250 & 0.0570 & 0.0488 & 0.0647 & invg & Inf \\
\hline sigma_meta & 0.025 & 0.0033 & 0.0029 & 0.0036 & invg & Inf \\
\hline sigma_w_e & 0.050 & 0.0127 & 0.0109 & 0.0145 & invg & Inf \\
\hline sigma_w_ey & 0.050 & 0.0150 & 0.0129 & 0.0171 & invg & Inf \\
\hline
\end{tabular}

De forma resumida, um choque de $6 \%$ na cotação internacional do petróleo leva a um aumento de $0,6 \%$ na inflação headline e de cerca de $0,4 \%$ na inflação core logo após o choque, que rapidamente retornam ao estado estacionário. A resposta da política monetária varia a depender se o Banco Central responde a desvios na inflação headline ou na inflação core, sendo o aumento dos juros menor para o segundo cenário. A resposta contracionista gera uma queda de $0,12 \%$ a $0,18 \%$ no consumo trimestral das famílias de forma hump-shaped, notadamente diante do menor consumo de bens energéticos (queda de $2 \%$ ), mas também de bens não energéticos (queda de 0,06\% a $0,12 \%)$. No quadro abaixo pode-se encontrar um quadro-resumo de tais impactos (pico, ou variação máxima atingida), observados a partir das IRFs.

\begin{tabular}{|c|c|c|c|c|}
\hline \multirow{2}{*}{ Efoito nas variáveis no pico (variação máxima) } & \multicolumn{2}{|c|}{ Inflação headline } & \multicolumn{2}{|c|}{ Inflação core } \\
\hline & Forward-looking & Baekw ard-looking & Forward-looking & Backward-looking \\
\hline - consumo familias (c) & $\begin{array}{r}-0.15 \% \\
\end{array}$ & $\begin{array}{r}-0.18 \% \\
\end{array}$ & $\begin{array}{r}-0.12 \% \\
\end{array}$ & $-0.15 \%$ \\
\hline - salários (W) & $-0.45 \%$ & $-0.60 \%$ & $-0.40 \%$ & $-0.50 \%$ \\
\hline - taxa de juros nominal (i) & $0.16 \%$ & $0.12 \%$ & $0.12 \%$ & $0.06 \%$ \\
\hline - inflação headline (pi) & $0.60 \%$ & $0.60 \%$ & $0.60 \%$ & $0.60 \%$ \\
\hline - consumo nāo energéticos (c_n) & $-0.07 \%$ & $-0.12 \%$ & $-0.06 \%$ & $-0.10 \%$ \\
\hline - consumo derivados petróleo pelas familias (c_e) & $-2.00 \%$ & $-2.00 \%$ & $-2.00 \%$ & $-2.00 \%$ \\
\hline - inflação core (pi_n) & $0.40 \%$ & $0.40 \%$ & $0.40 \%$ & $0.40 \%$ \\
\hline - demandainsumos de petróleo pelas firmas ( $\mathrm{v}$-e $)$ & $-2.50 \%$ & $-2.50 \%$ & $-250 \%$ & $-2.50 \%$ \\
\hline - taxa de cambio real (s_real) & $-0.60 \%$ & $-0.60 \%$ & $-0.60 \%$ & $-0.60 \%$ \\
\hline Log verossimilhança marginal & 2277.25 & 2274.36 & 2276.06 & 2270.31 \\
\hline
\end{tabular}

Como pode ser visto, um Banco Central que responda a mudanças na inflação headline tende a causar um maior aumento na taxa de juros nominal e uma maior contração no consumo das famílias. Ainda, para um mesmo tipo 
de inflação, uma regra de política monetária forward-looking tende a incorrer em maiores aumentos na taxa de juros por menos tempo, por enxergar que o choque de inflação é de curta duração e, portanto, é menos contracionista.

Nas subseções seguintes, será analisado o impacto de três choques diferentes sobre a economia sob a regra de política monetária que mira a inflação headline de forma forward-looking, visto que foi a versão com maior log verossimilhança marginal. Apesar de tal fato significar uma melhor aderência aos dados, uma diferença tão pequena entre as diferentes alternativas não seria suficiente para afirmar que o Banco Central do Brasil teria reagido de tal forma no período analisado. As posteriores e IRFs das demais alternativas podem ser encontradas no Apêndice. Ainda um teste de robustez, com base na estimação do modelo com a série de dados de 2005 a 2019, período onde a meta de inflação ficou praticamente constante no Brasil, pode ser encontrado no Apêndice.

\section{1}

\section{Choque de política monetária}

Um choque de política monetária, como pode ser observado na figura abaixo, de acordo com o modelo estimado, geraria uma maior taxa de juros nominal (i). A política monetária mais restritiva, portanto, leva a uma redução da inflação (pi) e dos preços (p).

Há também uma menor demanda por trabalho (l), que implica em menores salários (w), diante de menor demanda para o mesmo nível de oferta, que por si implica em menor custo marginal (mc) para as firmas.

Esse choque de política monetária, com aumento da taxa de juros doméstica, gera uma valorização do câmbio (s), respeitando a Paridade Descoberta da Taxa de Juros, que pode ser justificada por conta da maior atratividade da moeda local para investidores internacional, gerando maior demanda pelo real.

O choque de política monetária gera menor consumo (c) por parte das famílias, com redução da demanda agregada por conta de menores incentivos para consumir e maiores incentivos a poupar, por conta da maior taxa de juros, apesar de um atingimento do pico da redução alguns trimestres após o choque por conta da persistência de hábito.

O choque de política monetária atua por dois canais, em sentidos opostos, na demanda pelo insumo importado (z). Positivamente, na medida em que insumos importados ficam mais baratos por conta da valorização da taxa de câmbio doméstica, e negativamente, na medida em que há uma redução na demanda agregada. Aparentemente, pelo gráfico, o efeito renda é dominante, gerando redução na demanda por insumos importados. Nas variáveis energéticas, devido à valorização cambial, há redução no preço dos 
derivados de petróleo nacionais $\left(p_{\_} e\right)$, o que impulsiona aumento de sua demanda por parte das famílias $\left(c \_e\right)$, apesar de posterior redução devido à queda no consumo das famílias de forma hump-shaped. O mesmo formato ocorre para a demanda por derivados por parte das firmas $\left(v \_e\right)$.

Em termos de timing, a maioria das variáveis está de volta ao estado estacionário em 2,5 anos (10 trimestres), compatível com os resultados de Castro et al (2011). 
IRF: Choque de política monetária
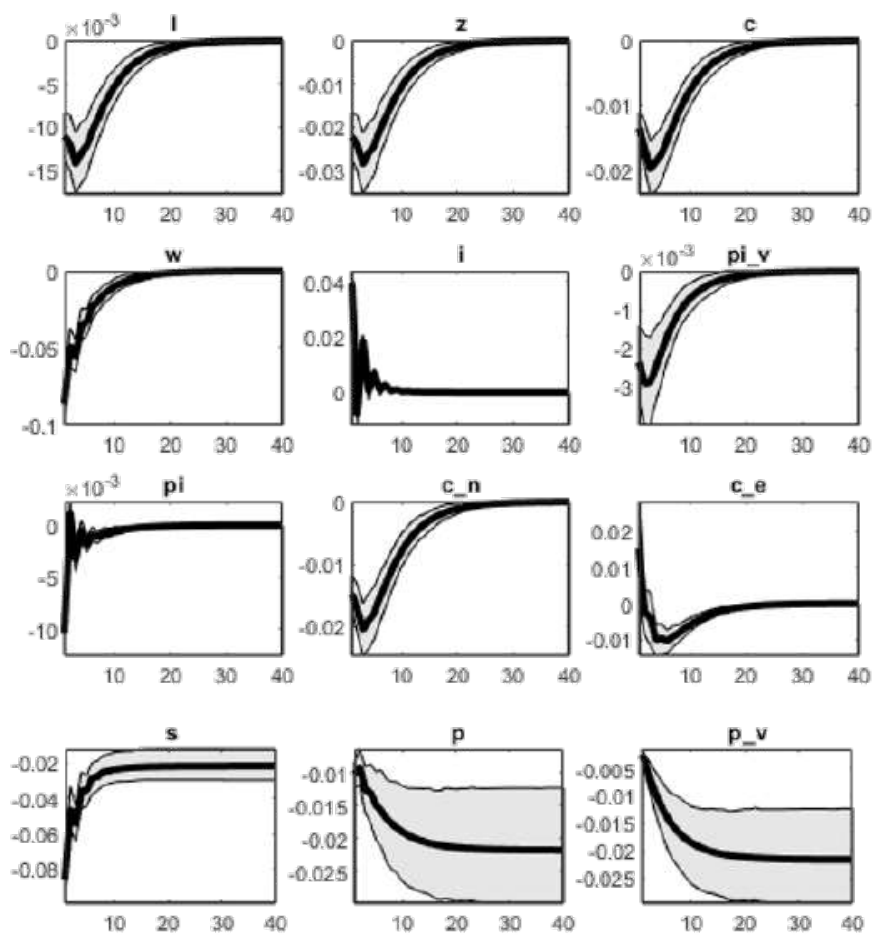

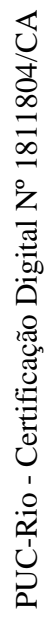
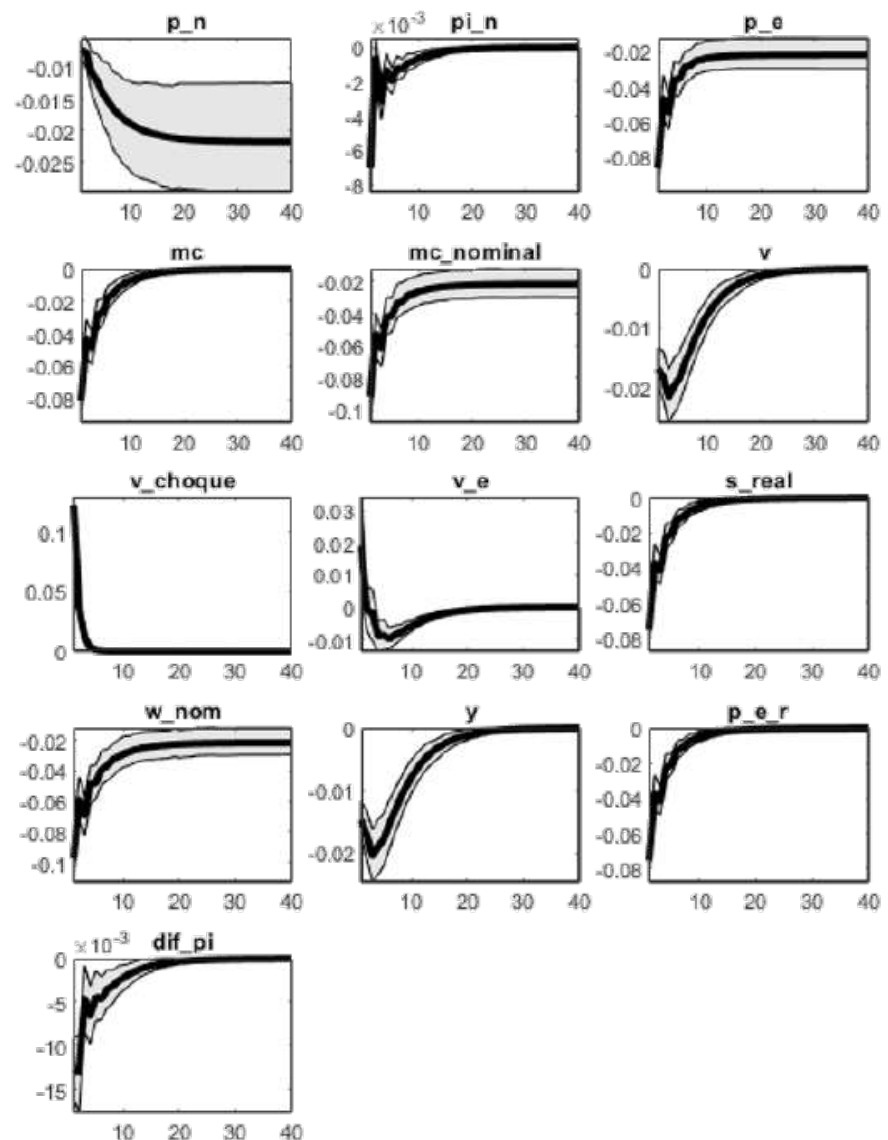


\section{Choque nos preços internacionais do petróleo}

Um choque nos preços internacionais de petróleo gera um aumento no preço de derivados nacionais e, consequentemente, uma menor demanda por derivados pelas famílias e pelas firmas produtoras de bens finais.

O aumento do custo das firmas gera um maior preço dos produtos finais e da inflação headline e core no curtíssimo prazo, rapidamente voltando ao steady state. A resposta do Banco Central ao aumentar a taxa de juros, por outro lado, se dá de forma mais persistente.

O efeito renda do maior preço de derivados de petróleo sobre as famílias, aliado aos menores salários, geram uma queda no consumo das famílias, de forma hump-shaped por conta da persistência de hábitos e, portanto, apresenta características de um choque contracionista sobre a economia.

Ainda, a taxa de câmbio real aprecia, impulsionada pela maior taxa de juros nacional segundo a Paridade Descoberta da Taxa de Juros. 
IRF: Choque nos preços internacionais de petróleo
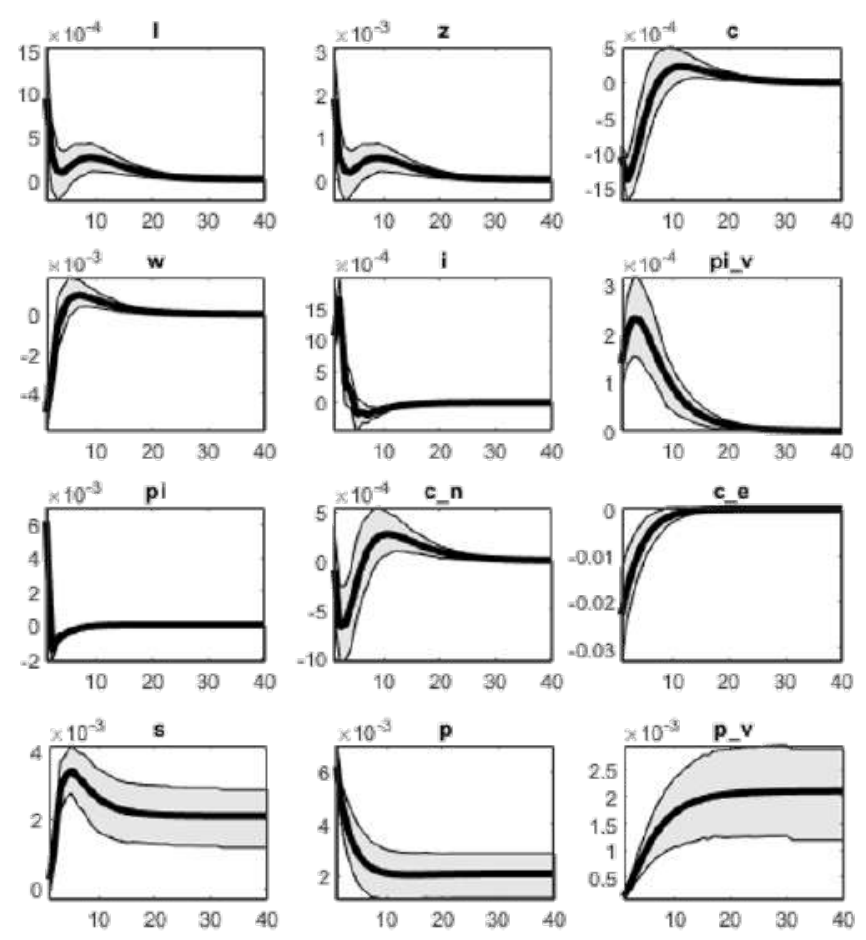

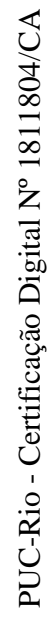
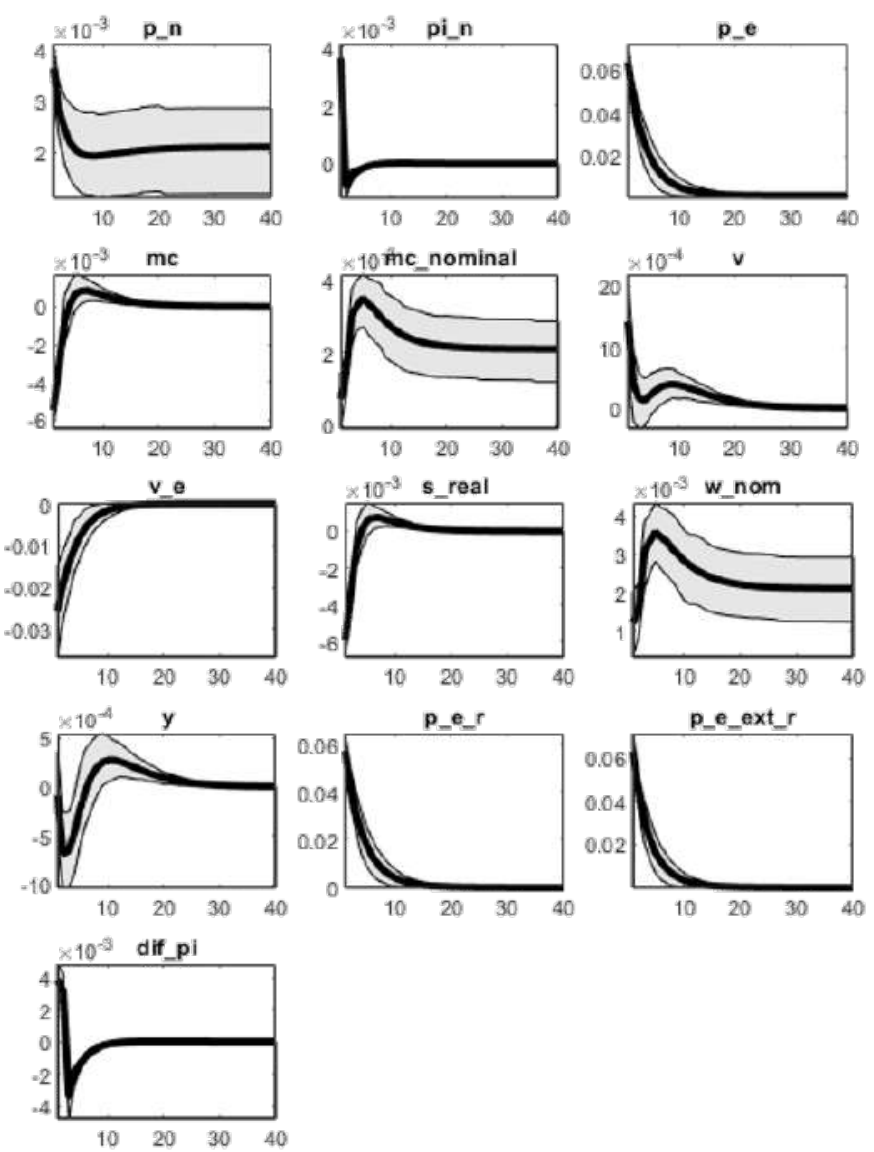


\section{3}

\section{Choque nos preços nacionais de derivados de petróleo}

$\mathrm{Na}$ mesma linha do choque de preço de petróleo internacional, um choque no preço de derivados nacionais gera um choque contracionista sobre a economia, com aumento da taxa de juros nominal de forma mais persistente do que o aumento da inflação. Ainda, a redução do consumo das famílias ocorre de forma hump-shaped. 
IRF: Choque nos preços derivados nacionais
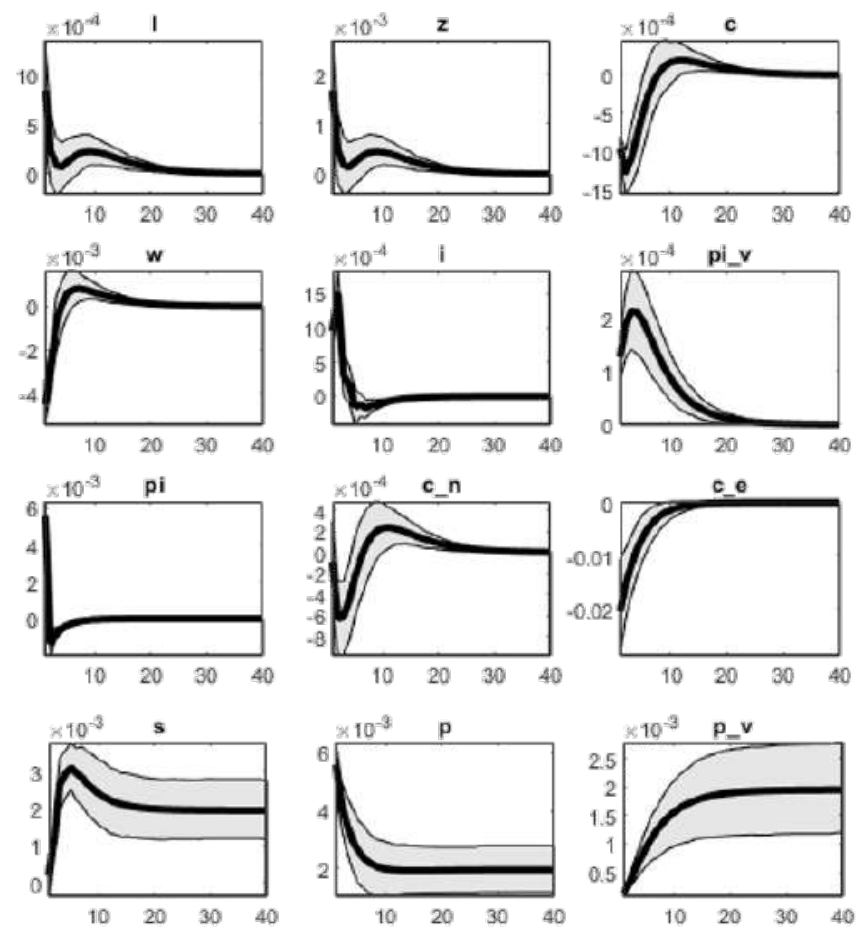

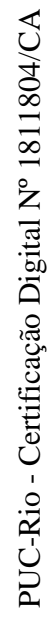
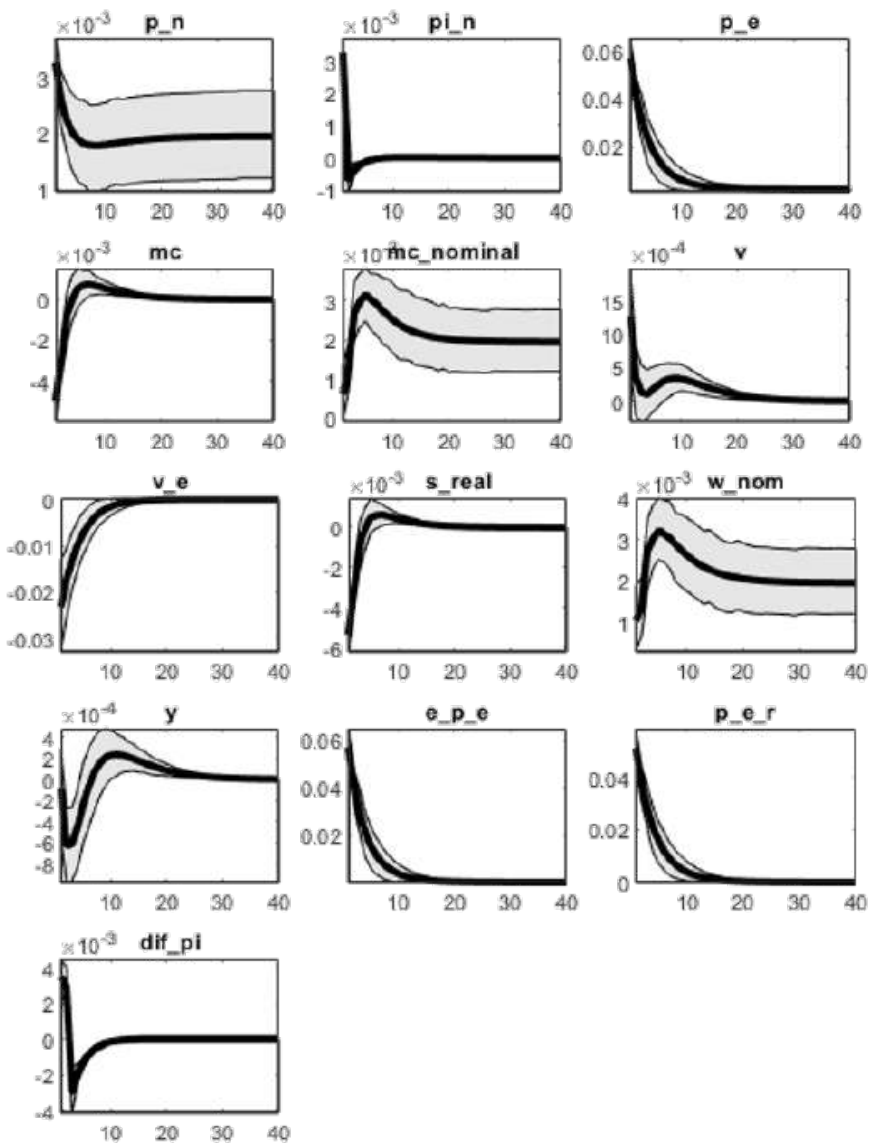


\section{5}

\section{Conclusão}

Mudanças no preço de petróleo são fonte importante para flutuações econômicas, assim como uma proxy para choques globais, por afetar diversas economias simultaneamente.

A inovação do modelo apresentado está na existência de bens energéticos, aqui representados por derivados de petróleo. Estes são utilizados como insumo para a produção do bem final não energético, além de entrarem diretamente na cesta de consumo das famílias, o que poderia refletir o uso de gasolina para deslocamento, profissional e de lazer, e o uso de GLP para cozimento de alimentos.

Neste modelo, um choque de $6 \%$ na cotação internacional do petróleo leva a um aumento de $0,6 \%$ na inflação headline e de cerca de $0,4 \%$ na inflação core logo após o choque, que rapidamente retornam ao estado estacionário. A resposta da política monetária varia a depender se o Banco Central responde a desvios na inflação headline ou na inflação core, sendo o aumento dos juros menor para o segundo cenário. Ainda, uma regra de política monetária forward-looking tende a incorrer em maiores aumentos na taxa de juros por menos tempo, sendo menos contracionista, por enxergar que o choque de inflação é de curta duração. A resposta contracionista gera uma queda de $0,12 \%$ a $0,18 \%$ no consumo trimestral das famílias de forma hump-shaped, notadamente diante do menor consumo de bens energéticos (queda de 2\%), mas também de bens não energéticos (queda de 0,06\% a 0,12\%).

Entre os potenciais estudos futuros estão um melhor refinamento do setor energético, incluindo oferta e demanda nacional e estrangeira, e a adaptação para a realidade brasileira como relevante produtor de petróleo, o que faria com que choques positivos no preço de petróleo possam ter também um impacto estimulativo na economia a partir do canal de aumento de investimentos, e não apenas contracionista como foi mostrado no estudo.

Ainda, em um contexto global de mudanças climáticas e transição para uma economia de baixo carbono, faz-se mister entender de que forma choques climáticos sistêmicos e a maior participação de fontes renováveis na matriz energética poderão afetar a macroeconomia e a eficácia da resposta da política monetária. 


\section{6}

\section{Referências bibliográficas}

[Agência Nacional de Petróleo, Gás Natural e Biocombustíveis/ANP, 2019]

MME/ANP. Anuário Estatístico ANP, 2019. 3.1

[An e Kang, 2011] AN, S.; KANG, H.. Oil Shocks in a DSGE Model

for the Korean Economy. In: Commodity Prices and Markets, NBER

Chapters, p. 295-321. National Bureau of Economic Research, Inc, June 2011. 4

[Blanchard e Gali, 2007] BLANCHARD, O. J.; GALI, J.. The Macroeconomic Effects of Oil Shocks. Why are the 2000s So Different from the 1970s? Número 13368. 2007. 1

[Bodenstein et al, 2008] BODENSTEIN, M.; ERCEG, C. J. ; GUERRIERI, L.. Optimal monetary policy with distinct core and headline inflation rates. FRB International Finance Discussion, (941), 2008. 1

[Bodenstein et al, 2010] BODENSTEIN, M.; ERCEG, C. ; GUERRIERI, L.. Oil shocks and external adjustment. Board of Governors of the Federal Reserve System - International Finance Discussion Papers, (897R), 2010. 1

[Carvalho e Vilela, 2015] CARVAlHO, C. V.; VILELA, A. D.. What if Brazil Hadn't Floated the Real in 1999? Textos para discussão 647, Department of Economics PUC-Rio (Brazil), Nov. 2015. 4

[Castro et al, 2011] CASTRO, M. R.; GOUVEA, S. N.; MINELLA, A.; SANTOS, R. C. ; SOUZA-SOBRINHO, N. F.. Samba: Stochastic analytical model with a bayesian approach. Working Papers 235, Banco Central do Brasil, 2011. 1

[Cristiano et al, 2005] CHRISTIANO, L.; EICHENBAUM, M. ; EVANS, C.. Nominal rigidities and the dynamic effects of a shock to monetary policy. Journal of Political Economy, 113(1):1-45, 2005. 1

[Empresa de Pesquisa Eenergética/MME, 2019] MME/EPE. Plano Decenal de Expansão de Energia 2029, 2019. 3.1

[Hamilton, 1983] HAMILTON, J.. Oil and the macroeconomy since world war ii. Journal of Political Economy, 91:228-48, 02 1983. 1 
[Linardi, 2016] LINARDI, F.. Assessing the Fit of a Small OpenEconomy DSGE Model for the Brazilian Economy. Working Papers Series 424, Central Bank of Brazil, Research Department, 2016. 3.3

[Medina e Soto, 2005] MEDINA, J. P.; SOTO, C.. Oil Shocks and Monetary Policy in an Estimated DSGE Model for a Small Open Economy. Working Papers Central Bank of Chile 353, Central Bank of Chile, Dec. 2005. 1

[Rasche e Tatom, 1981] RASCHE, R. H.; TATOM, J. A.. Energy price shocks, aggregate supply and monetary policy: The theory and the international evidence. Carnegie-Rochester Conference Series on Public Policy, 14:9-93, 1981. 1

[Smets e Wouters, 2003] SMETS, F.; WOUTERS, R.. An estimated stochastic dynamic general equilibrium model of the euro area. Journal of the European Economic Association, 1(5):1123-1175, 2003. 1

[Wu e Xia, 2016] WU, J. C.; XIA, F. D.. Measuring the macroeconomic impact of monetary policy at the zero lower bound. Journal of Money, Credit and Banking, 48(2-3):253-291, 2016. 3.1 


\section{Apêndice}

\section{1}

\section{Equações log linearizadas}

$$
\begin{aligned}
& \pi_{v}=\left(\frac{\beta}{1+(\beta * \gamma)} * E_{0}\left(\pi_{v, t+1}\right)+\frac{\lambda *(1-\beta *(1-\lambda))}{1+(\beta * \gamma)) *(1-\lambda)} *\left(m c_{n}-p_{v}+m u\right)+\frac{\gamma}{(1+(\beta * \gamma))} * \pi_{v, t-1}\right. \\
& \pi_{v, t}=p_{v, t}-p_{v, t-1} \\
& c_{t}-\frac{h}{(1+h)} * c_{t-1}=\frac{1}{(1+h)} * E_{0}\left(c_{t+1}\right)-\frac{(1-h)}{\sigma *(h+1)} *\left(i_{t}-E_{0}\left(\pi_{t+1}\right)+g_{t}\right) \\
& m c_{t}=m c_{n_{t}}-p_{t} \\
& m c_{t}=(1-\delta) * w_{t}-a_{t}+\delta *\left(s_{t}+p_{t}^{*}-p_{t}\right) \\
& w_{t}=\frac{\sigma}{1-h} * c_{t}-\frac{\sigma * h}{1-h} * c_{t-1}+\varphi * l_{t} \\
& l_{t}=\delta *\left(s_{t}+p_{t}^{*}-w_{t}-p_{t}\right)+v_{t}-a_{t} \\
& z_{t}=w_{t}-\left(s_{t}+p_{t}^{*}-p_{t}\right)+l_{t} \\
& s_{t}^{r}=s_{t}+p_{t}^{*}-p_{t} \\
& w_{t}=w_{t}^{n}-p_{t}
\end{aligned}
$$

$p_{e_{t}}^{r}=p_{e_{t}}^{*_{r}}+s_{t}^{r}+\epsilon_{t}^{p_{e}}$

$y_{t}=c_{n, t}$

Cesta de consumo das famílias e seu preço:

$$
\begin{aligned}
& \hat{c}_{n, t}=\rho_{e} \hat{p}_{t}-\rho_{e} \hat{p}_{n, t}+\hat{c}_{t}-w_{e} \hat{w}_{e, t} \\
& \hat{c}_{e, t}=\rho_{e} \hat{p}_{t}-\rho_{e} \hat{p}_{e, t}+\hat{c}_{t}+\hat{w}_{e, t} \\
& \hat{w}_{e, t}=\rho_{w_{e, t}} \hat{w}_{e, t}+\sigma_{w_{e, t}} \varepsilon_{t}^{w_{e}} \\
& \hat{p}_{t}=\left(1-w_{e}\right) \hat{p}_{n, t}+\left(w_{e}\right) \hat{p}_{e, t}
\end{aligned}
$$

Cesta de insumos das firmas produtoras de bens finais e seu preço: $\hat{v}_{t}=\rho_{e_{y}} \hat{p}_{n, t}-\rho_{e_{y}} \hat{p}_{v, t}+\hat{c}_{n, t}-w_{e_{y}} \hat{w}_{e y, t}$ 


$$
\begin{aligned}
& \hat{v}_{e, t}=\rho_{e_{y}} \hat{p}_{n, t}-\rho_{e_{y}} \hat{p}_{e, t}+\hat{c}_{n, t}+\hat{w}_{e_{y}, t} \\
& w_{e_{y}, t}=\rho_{w_{e y}, t} w_{e_{y}, t-1}+\sigma_{w_{e y}, t} \varepsilon_{t}^{w_{e y}} \\
& \hat{p}_{n, t}=\left(1-w_{e_{y}}\right) \hat{p}_{v, t}+w_{e_{y}} \hat{p}_{e, t}
\end{aligned}
$$

\section{2}

\section{Derivação da cesta de consumo das famílias ótima e seu preço}

Arrumando a equação para facilitar a derivação:

$$
C_{t}=\left[\left(1-w_{e, t}\right)^{\frac{1}{\rho_{e}}} C_{n, t}^{\frac{\rho_{e}-1}{\rho_{e}}}+\left(w_{e, t}\right)^{\frac{1}{\rho_{e}}} C_{e, t}^{\frac{\rho_{e}-1}{\rho_{e}}}\right]^{\frac{\rho_{e}}{\rho_{e}-1}}
$$

Maximização do "lucro"do consumidor criando sua cesta final:

$$
\max P_{t} * C_{t}-P_{n, t} * C_{n, t}-P_{e, t} * C_{e, t}
$$

Sujeito a:

$$
C_{t}=\left[\left(1-w_{e, t}\right)^{\frac{1}{\rho_{e}}} C_{n, t}^{\frac{\rho_{e}-1}{\rho_{e}}}+\left(w_{e, t}\right)^{\frac{1}{\rho_{e}}} C_{e, t}^{\frac{\rho_{e}-1}{\rho_{e}}}\right]^{\frac{\rho_{e}}{\rho_{e}-1}}
$$

Igual a:

$$
\max P_{t} *\left[\left(1-w_{e, t}\right)^{\frac{1}{\rho_{e}}} C_{n, t}^{\frac{\rho_{e}-1}{\rho_{e}}}+\left(w_{e, t}\right)^{\frac{1}{\rho_{e}}} C_{e, t}^{\frac{\rho_{e}-1}{\rho_{e}}}\right]^{\frac{\rho_{e}}{\rho_{e}-1}}-P_{n, t} * C_{n, t}-P_{e, t} * C_{e, t}
$$

Suponha $X_{t}$ :

$$
X_{t}=\left[\left(1-w_{e, t}\right)^{\frac{1}{\rho_{e}}} C_{n, t}^{\frac{\rho_{e}-1}{\rho_{e}}}+\left(w_{e, t}\right)^{\frac{1}{\rho_{e}}} C_{e, t}^{\frac{\rho_{e}-1}{\rho_{e}}}\right]^{\frac{1}{\rho_{e}-1}}
$$

Derivando em relação a $C_{n, t}$ :

$$
\frac{\rho_{e}}{\rho_{e}-1} P_{t} X_{t}\left(1-w_{e, t}\right)^{\frac{1}{\rho_{e}}} C_{n, t}^{\frac{-1}{\rho_{e}}} \frac{\left(\rho_{e}-1\right)}{\rho_{e}}=P_{n, t}
$$

Assim:

$$
C_{n, t}={\frac{P_{t}}{P_{n, t}}}^{\rho_{e}}\left(1-w_{e, t}\right) C_{t}
$$

O mesmo vale para $C_{e, t}$

$$
C_{e, t}={\frac{P_{t}}{P_{e, t}}}^{\rho_{e}}\left(w_{e, t}\right) C_{t}
$$

Alterando a forma das equações para o mais comumente utilizado na literatura:

$$
\begin{gathered}
C_{n, t}=\left(\frac{P_{n, t}}{P_{t}}\right)^{-\rho_{e}}\left(1-w_{e, t}\right) C_{t} \\
C_{e, t}=\left(\frac{P_{e, t}}{P_{t}}\right)^{-\rho_{e}}\left(w_{e, t}\right) C_{t}
\end{gathered}
$$

Substituindo $C_{n, t}$ e $C_{e, t}$ em $C_{t}$ :

$$
C_{t}=\left[\left(1-w_{e, t}\right)^{\frac{1}{\rho_{e}}-\frac{1}{\rho_{e}}+1} C_{t}^{\frac{\rho_{e}-1}{\rho_{e}}}\left(\frac{P_{t}}{P_{n, t}}\right)^{\rho_{e}-1}+w_{e, t}^{\frac{1}{\rho_{e}}-\frac{1}{\rho_{e}}+1} C_{t}^{\frac{\rho_{e}-1}{\rho_{e}}}\left(\frac{P_{t}}{P_{e, t}}\right)^{\rho_{e}-1}\right]^{\frac{\rho_{e}}{\rho_{e}-1}}
$$

Cortando $C_{t}$ e isolando $P_{t}$ :

$$
P_{t}=\left[\left(1-w_{e, t}\right) P_{n, t}^{1-\rho_{e}}+w_{e, t} P_{e, t}^{1-\rho_{e}}\right]^{\frac{1}{1-\rho_{e}}}
$$

O mesmo que:

$$
P_{t}^{1-\rho_{e}}=\left(1-w_{e, t}\right) P_{n, t}^{1-\rho_{e}}+w_{e, t} P_{e, t}^{1-\rho_{e}}
$$




\subsection{1}

\section{Log-Linearização}

$L O G$ em $C_{n, t}$ e $C_{e, t}:$

$$
c_{n, t}=\rho_{e} p_{t}-\rho_{e} p_{n, t}+c_{t}+\log \left(1-w_{e, t}\right)
$$

Diminuindo estado estacionário (S.S) e fazendo a seguinte aproximação $\log \left(1-w_{e, t}\right) \simeq-w_{e, t}$, já que $w_{e, t}$ é um valor baixo:

$$
\hat{c}_{n, t}=\rho_{e} \hat{p}_{t}-\rho_{e} \hat{p}_{n, t}+\hat{c}_{t}-w_{e, t}+w_{e}
$$

Supondo que a variação em torno do estado estacionário de $w_{e, t}$ segue um processo $\operatorname{AR}(1)$ :

$$
\hat{w}_{e, t}=\rho_{w_{e}} \hat{w}_{e, t}+\sigma_{w_{e}} \epsilon_{t}^{w_{e}}
$$

Chegamos então à equação final para $\hat{c}_{n, t}$ :

$$
\hat{c}_{n, t}=\rho_{e} \hat{p}_{t}-\rho_{e} \hat{p}_{n, t}+\hat{c}_{t}-w_{e} \hat{w}_{e, t}
$$

E para $\hat{c}_{e, t}$ :

$$
\hat{c}_{e, t}=\rho_{e} \hat{p}_{t}-\rho_{e} \hat{p}_{e, t}+\hat{c}_{t}+\hat{w}_{e, t}
$$

Para a equação de preços é necessário usar aproximação de primeira ordem de Taylor

Dividindo a equação de preços por $P_{t}$ :

$$
1=\left(1-w_{e, t}\right)\left(\frac{P_{n, t}}{P_{t}}\right)^{1-\rho_{e}}+w_{e, t}\left(\frac{P_{e, t}}{P_{t}}\right)^{1-\rho_{e}}
$$

Primeira ordem de taylor para $\left(\frac{P_{n, t}}{P_{t}}\right)^{1-\rho_{e}}$ :

$$
\begin{gathered}
\left(1-w_{e, t}\right)\left(\frac{P_{n, t}}{P_{t}}\right)^{1-\rho_{e}} \simeq\left(1-w_{e}\right)\left(\left(\frac{P_{n}}{P}\right)^{1-\rho_{e}}+\left(1-\rho_{e}\right)\left(\frac{P_{n}}{P}\right)^{1-\rho_{e}} \frac{P_{n, t}-P_{n}}{P_{n}}-(1-\right. \\
\left.\left.\rho_{e}\right)\left(\frac{P_{n}}{P}\right)^{1-\rho_{e}} \frac{\left(P_{t}-P\right)}{P}\right)-\left(\frac{P_{n}}{P}\right)^{1-\rho_{e}}\left(w_{e, t}-w_{e}\right) \\
\left(1-w_{e, t}\right)\left(\frac{P_{n, t}}{P_{t}}\right)^{1-\rho_{e}} \simeq\left(1-w_{e}\right)\left(\left(\frac{P_{n}}{P}\right)^{1-\rho_{e}}+\left(1-\rho_{e}\right)\left(\frac{P_{n}}{P}\right)^{1-\rho_{e}} \hat{p}_{n, t}-(1-\right. \\
\left.\left.\rho_{e}\right)\left(\frac{P_{n}}{P}\right)^{1-\rho_{e}} \hat{p}_{t}\right)-\left(\frac{P_{n}}{P}\right)^{1-\rho_{e}}\left(w_{e, t}-w_{e}\right)
\end{gathered}
$$

O mesmo vale para $\left(\frac{P_{e, t}}{P_{t}}\right)^{1-\rho_{e}}$ e portanto substituindo os termos de taylor na equação dos preços temos:

$$
\begin{gathered}
1=\left(1-w_{e}\right)\left(\frac{P_{n}}{P}\right)^{1-\rho_{e}}+w_{e}\left(\frac{P_{e}}{P}\right)^{1-\rho_{e}}+\left(1-w_{e}\right)\left(1-\rho_{e}\right)\left(\frac{P_{n}}{P}\right)^{1-\rho_{e}} \hat{p}_{n, t}-(1- \\
\left.w_{e}\right)\left(1-\rho_{e}\right)\left(\frac{P_{n}}{P}\right)^{1-\rho_{e}} \hat{p}_{t}+w_{e}\left(1-\rho_{e}\right)\left(\frac{P_{e}}{P}\right)^{1-\rho_{e}} \hat{p}_{e, t}-w_{e}\left(1-\rho_{e}\right)\left(\frac{P_{e}}{P}\right)^{1-\rho_{e}} \hat{p}_{t}- \\
\left(\frac{P_{n}}{P}\right)^{1-\rho_{e}}\left(w_{e, t}-w_{e}\right)+\left(\frac{P_{e}}{P}\right)^{1-\rho_{e}}\left(w_{e, t}-w_{e}\right)
\end{gathered}
$$


Sabendo que no estado estacionário:

$$
1=\left(1-w_{e}\right)\left(\frac{P_{n}}{P}\right)^{1-\rho_{e}}+w_{e}\left(\frac{P_{e}}{P}\right)^{1-\rho_{e}}
$$

Temos que:

$$
0=+\left(1-w_{e}\right)\left(\frac{P_{n}}{P}\right)^{1-\rho_{e}} \hat{p}_{n, t}-\left(1-w_{e}\right)\left(\frac{P_{n}}{P}\right)^{1-\rho_{e}} \hat{p}_{t}+w_{e}\left(\frac{P_{e}}{P}\right)^{1-\rho_{e}} \hat{p}_{e, t}-w_{e}\left(\frac{P_{e}}{P}\right)^{1-\rho_{e}} \hat{p}_{t}
$$

Dividindo a equação dos preços inicial por $P^{\left(1-\rho_{e}\right)}$ temos:

$$
\left(\frac{P_{t}}{P}\right)^{1-\rho_{e}}=\left(1-w_{e}\right)\left(\frac{P_{n, t}}{P}\right)^{1-\rho_{e}}+w_{e}\left(\frac{P_{e, t}}{P}\right)^{1-\rho_{e}}
$$

E avaliando no estado estacionário:

$$
1=\left(1-w_{e}\right)\left(\frac{P_{n}}{P}\right)^{1-\rho_{e}}+w_{e}\left(\frac{P_{e}}{P}\right)^{1-\rho_{e}}
$$

Sendo que $0<\frac{P n}{P} \leq 1$ e $0<\frac{P e}{P} \leq 1$

Solução:

$$
\frac{P n}{P}=1 \text { e } \frac{P e}{P}=1
$$

Voltando à log linearização:

$$
\begin{gathered}
0=\left(1-w_{e}\right) \hat{p}_{n, t}-\left(1-w_{e}\right) \hat{p}_{t}+\left(w_{e}\right) \hat{p}_{e, t}-\left(w_{e}\right) \hat{p}_{t} \\
\hat{p}_{t}=\left(1-w_{e}\right) \hat{p}_{n, t}+\left(w_{e}\right) \hat{p}_{e, t}
\end{gathered}
$$

\section{3}

Derivação e linearização da cesta de insumos das firmas produtoras de bens finais (simétrica ao consumo)

$$
Y_{t}=\left[\left(1-w_{e_{y}, t}\right)^{\frac{1}{\rho_{e}}} V_{t}^{\frac{\rho_{e_{y}}-1}{\rho_{e_{y}}}}+\left(w_{e_{y}, t}\right)^{\frac{1}{\rho_{e}}} V_{e, t}^{\frac{\rho_{e_{y}}-1}{\rho_{e}}}\right]^{\frac{\rho_{e y}}{\rho_{e y}-1}}
$$

Equilíbrio:

$$
Y_{t}=C_{n, t}
$$

Equações para produção:

$$
\begin{gathered}
\hat{v}_{t}=\rho_{e_{y}} \hat{p}_{n, t}-\rho_{e_{y}} \hat{p}_{v, t}+\hat{c}_{n, t}-w_{e_{y}} \hat{w}_{e_{y}, t} \\
\hat{v}_{e, t}=\rho_{e_{y}} \hat{p}_{n, t}-\rho_{e_{y}} \hat{p}_{e, t}+\hat{c}_{n, t}+\hat{w}_{e_{y}, t}
\end{gathered}
$$

E os preços:

$$
\begin{aligned}
\hat{p}_{n, t}-\hat{p}_{t} & =\left(1-w_{e_{y}}\right) \hat{p}_{v, t}+w_{e y} \hat{p}_{e, t}-\hat{p}_{t} \\
\hat{p}_{n, t} & =\left(1-w_{e_{y}}\right) \hat{p}_{v, t}+w_{e_{y}} \hat{p}_{e, t}
\end{aligned}
$$




\section{4}

Diagnósticos e gráficos para Alternativa Alternativa I: Forward-looking e headline inflation

Forward-looking e headline inflation:

MCMC diagnóstico multivariado
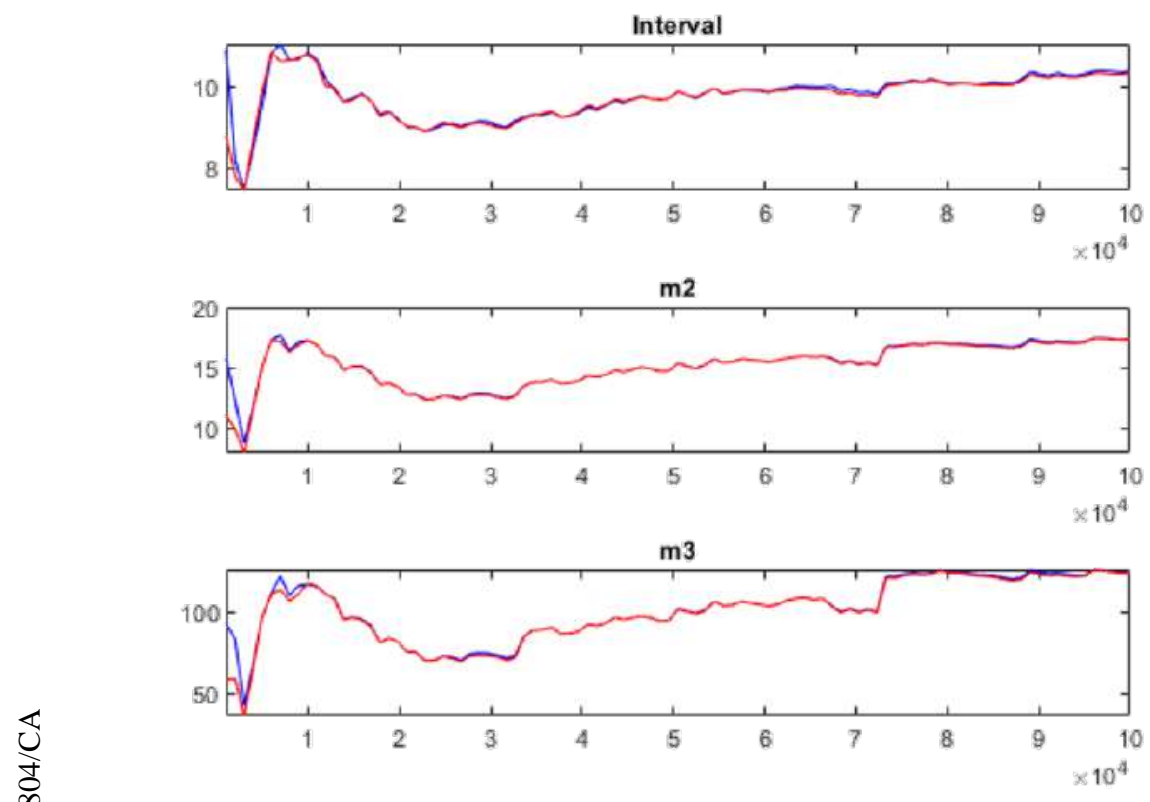

Historical x smoothed variables
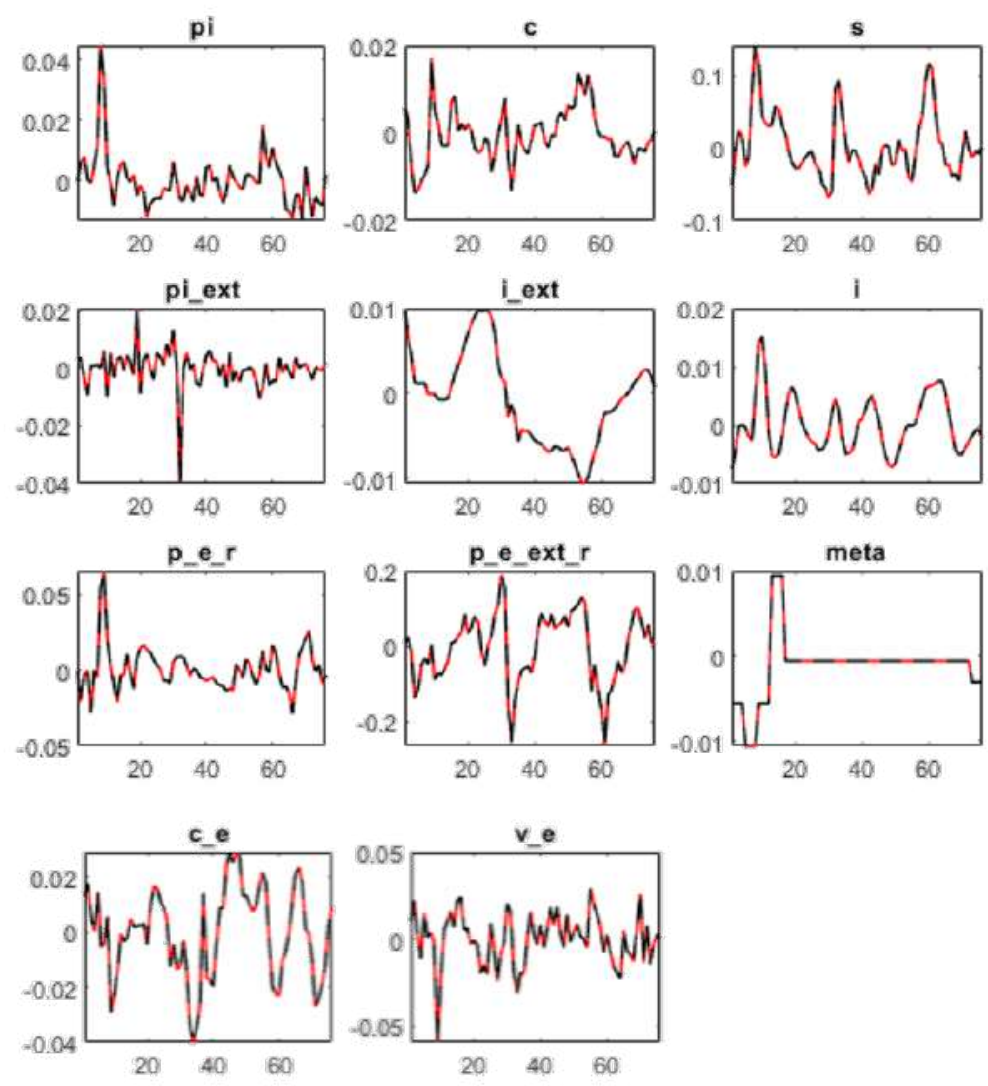
Smoothed shocks
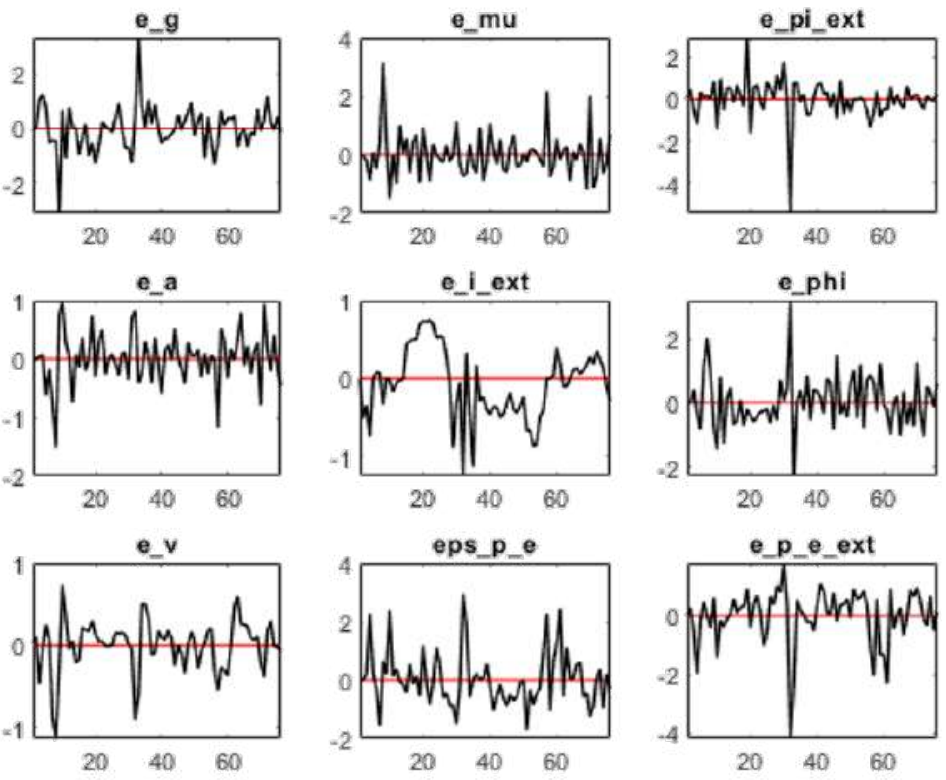

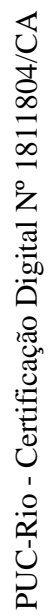
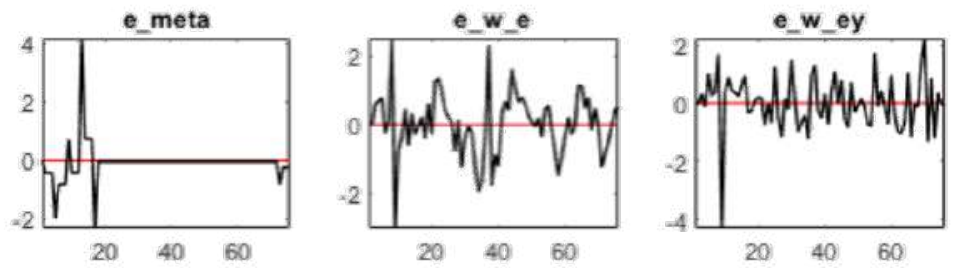
Forward-looking e headline inflation: Priors x posteriors
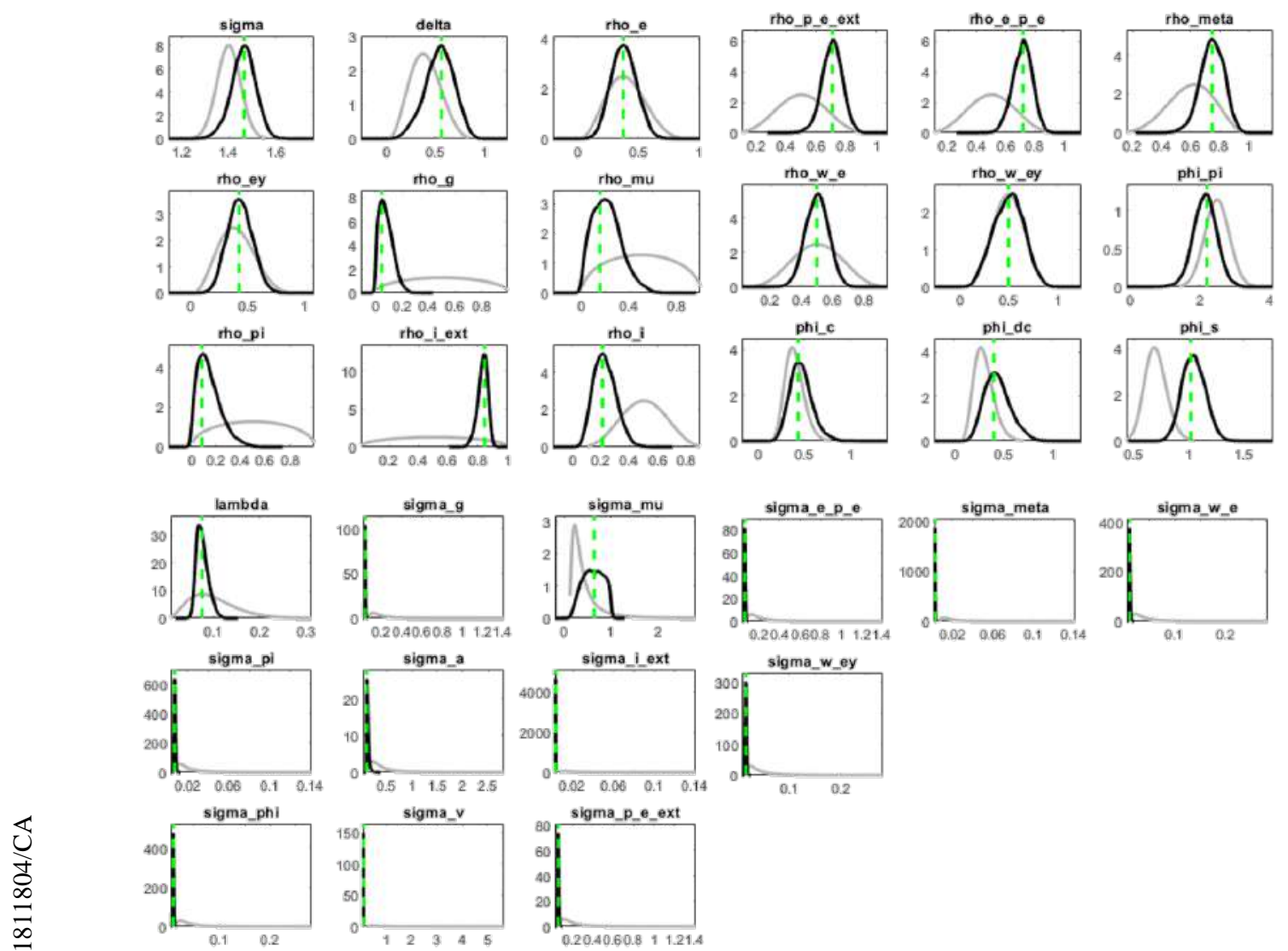

\subsection{1}

\section{Demais IRFs}

Abaixo são plotadas as IRFs para os demais choques que não foram analisadas anteriormente. 
IRF: Choque de produtividade
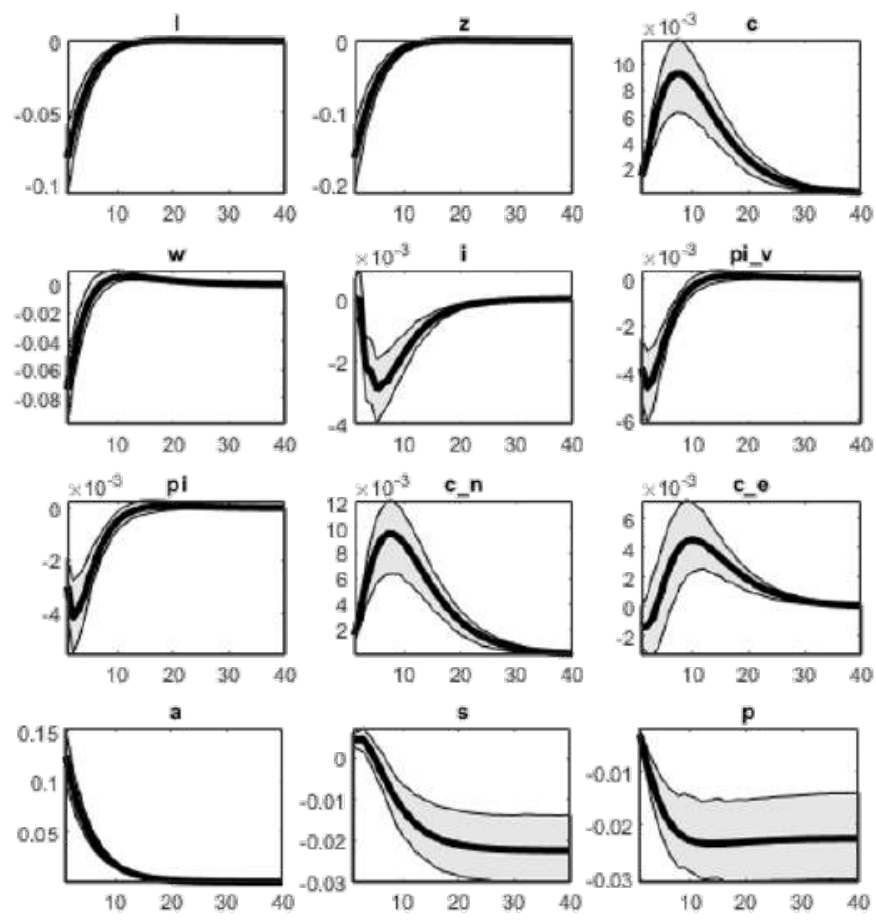

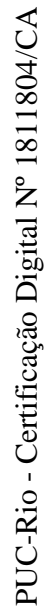
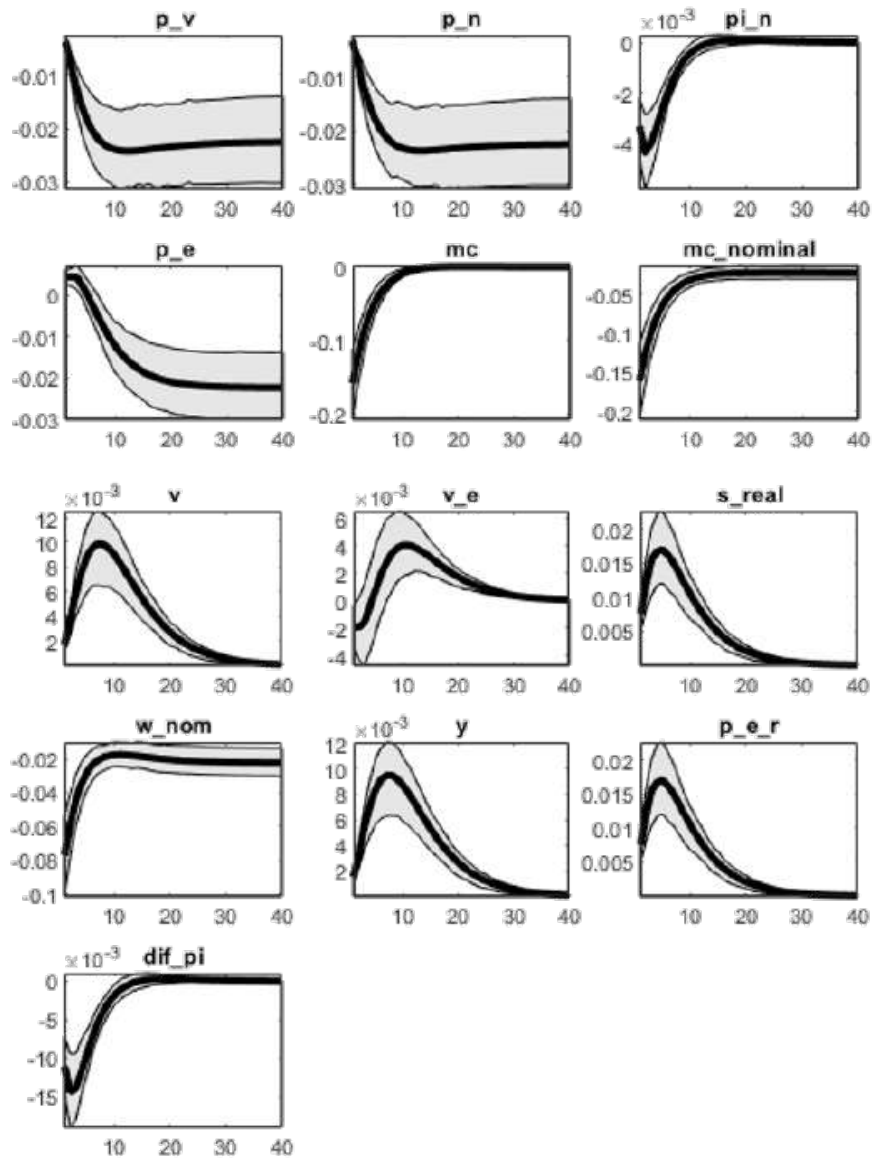
IRF: Choque na taxa de juros estrangeira
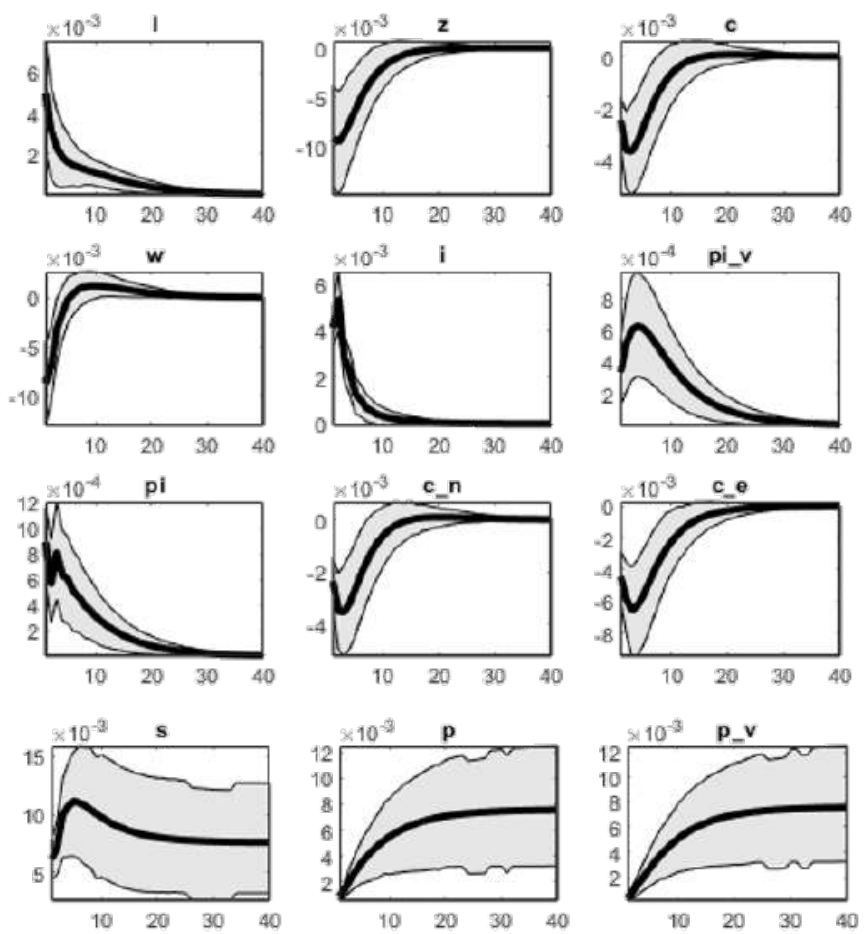

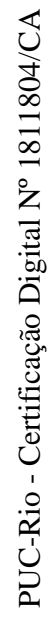
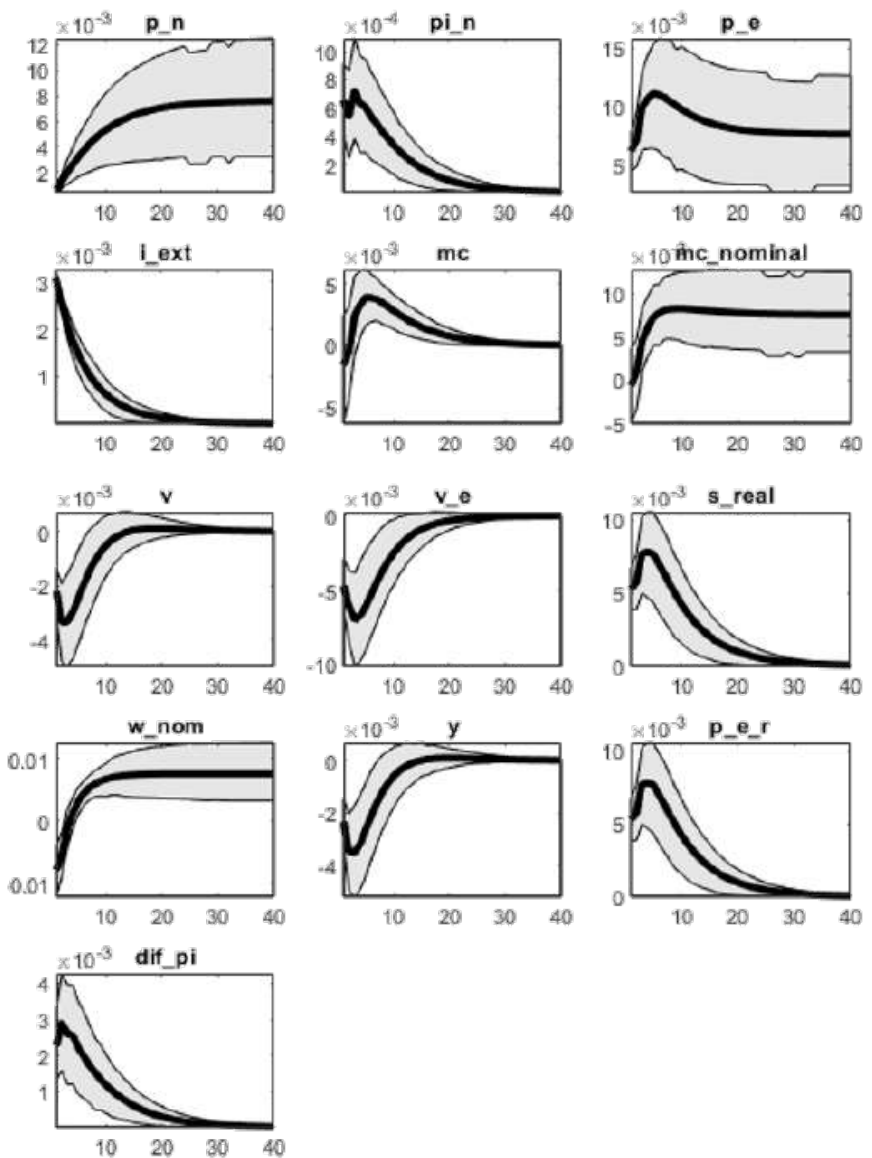
IRF: Choque de prêmio de risco
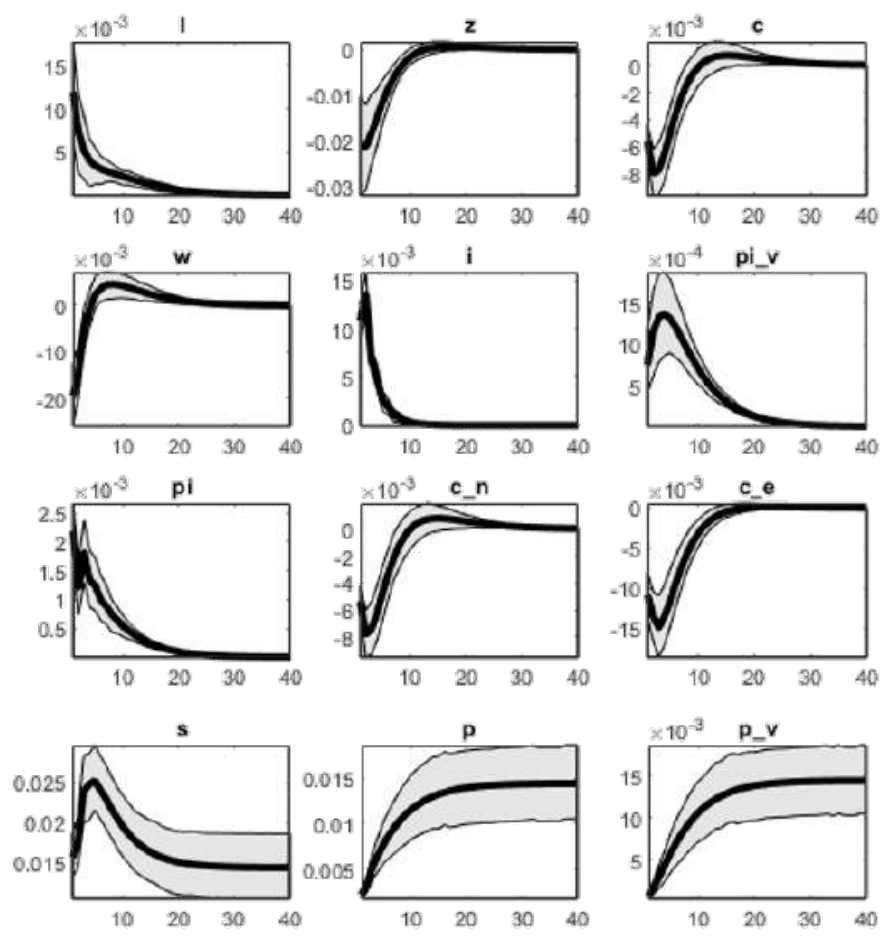

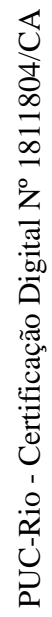
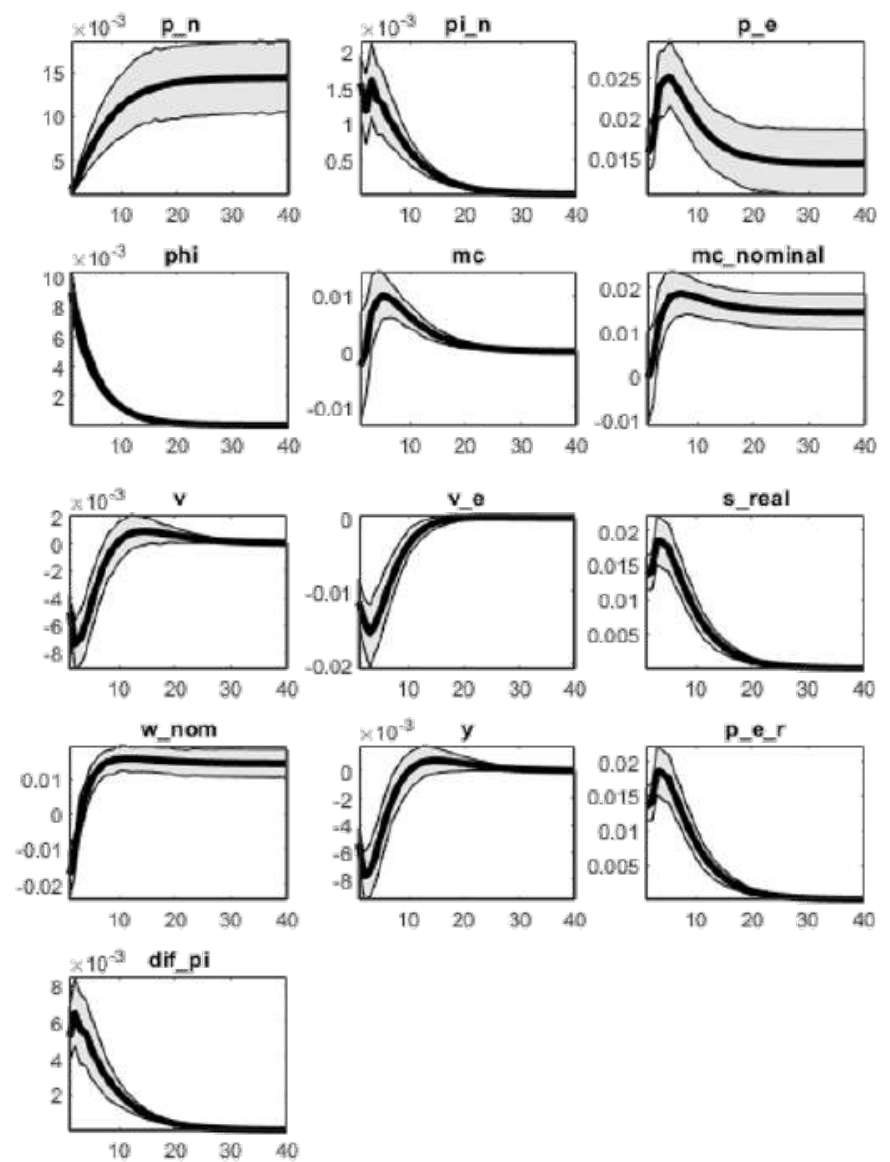


\section{5}

\section{Resultados da estimação de diferentes regras de política monetária}

Nas seções seguintes são apresentados os resultados das estimativas para as regras de política monetária alternativas, contendo o resultado do diagnóstico MCMC multivariado; os gráficos comparando as distribuições priors x posteriors; as variáveis históricas e suavizadas; os choques suavizados e as IRFs para alguns dos choques estimados.

\subsection{1}

\section{Alternativa II: Forward-looking e core inflation}

Forward-looking e core inflation: MCMC diagnóstico multivariado
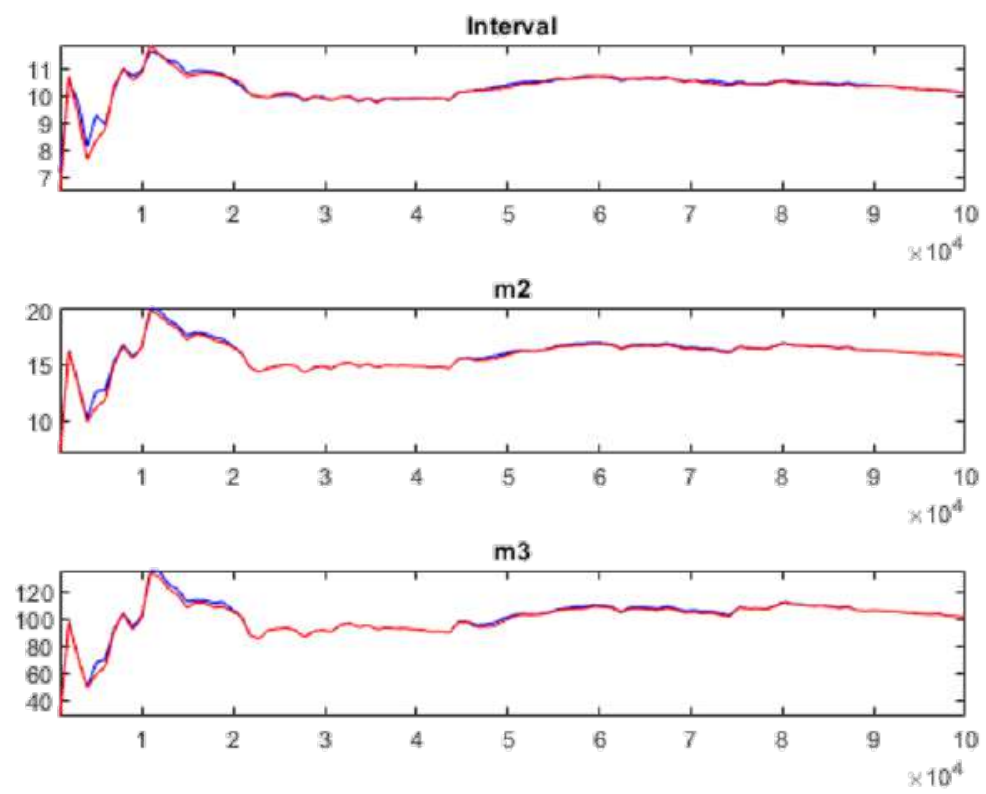
Historical x smoothed variables
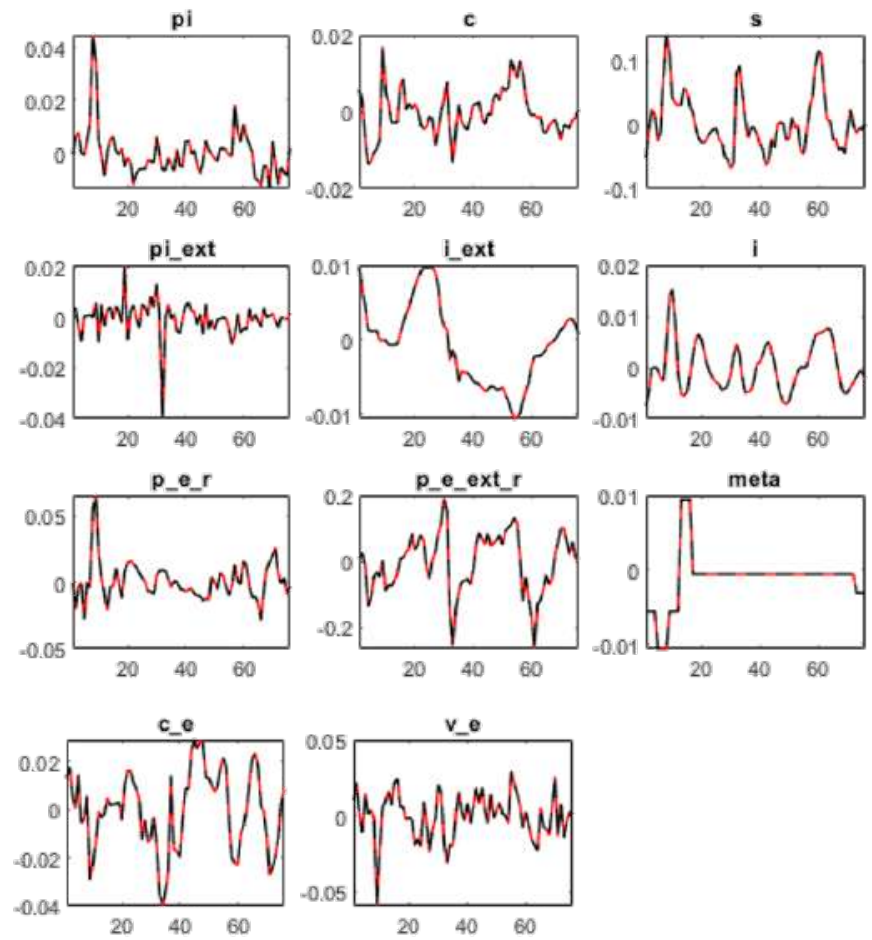

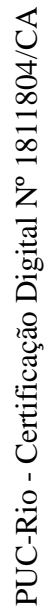

Smoothed shocks
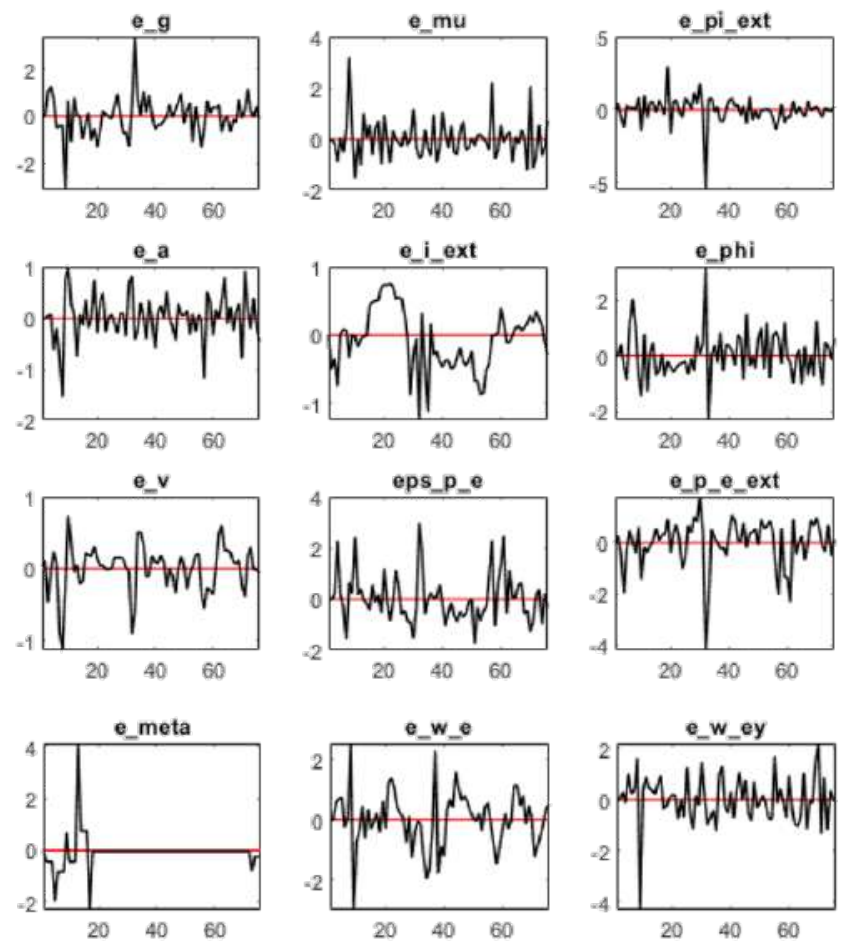
Forward-looking e core inflation: Priors x posteriors
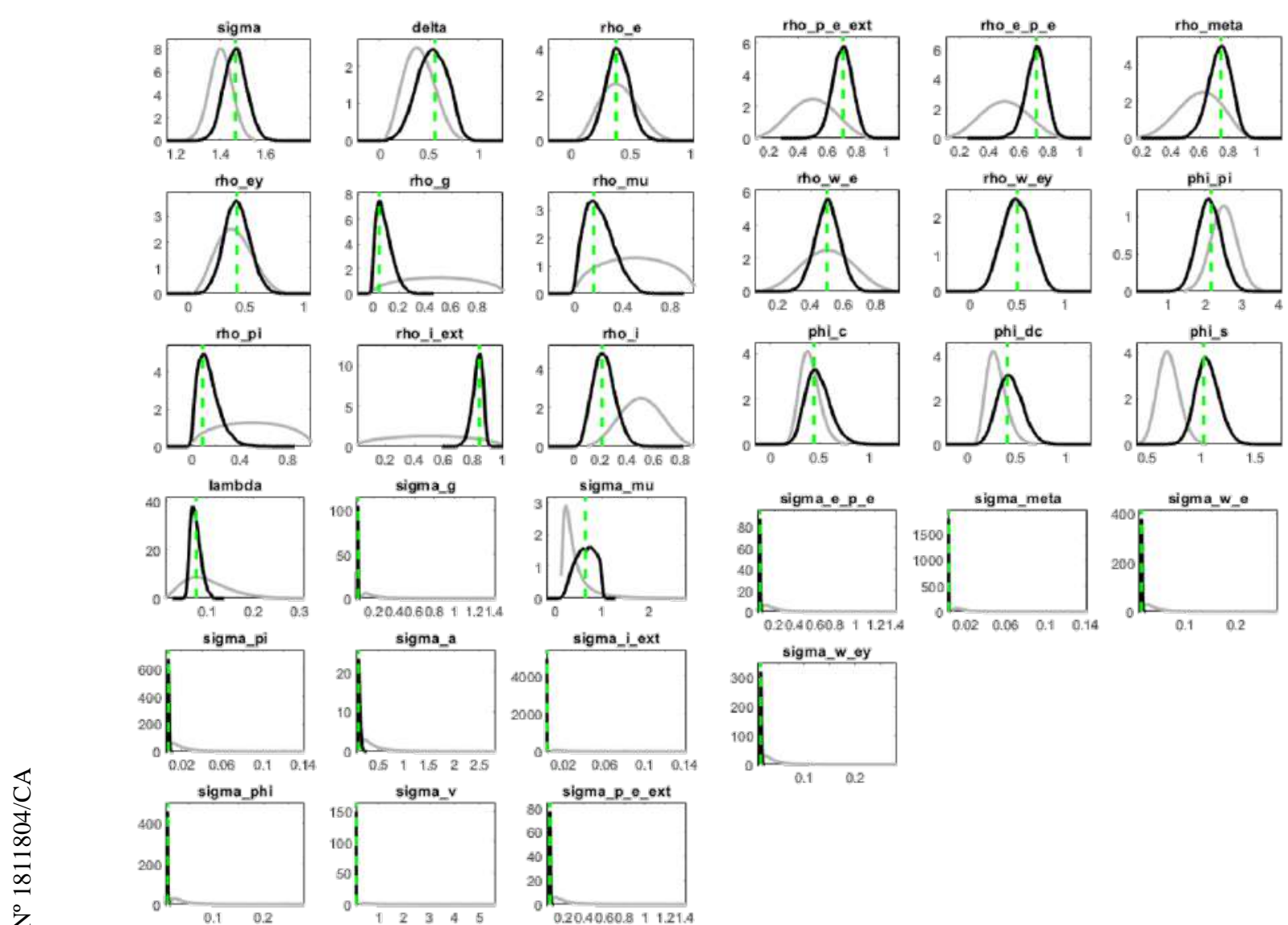

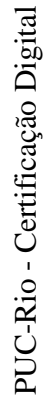


IRF: Choque de política monetária
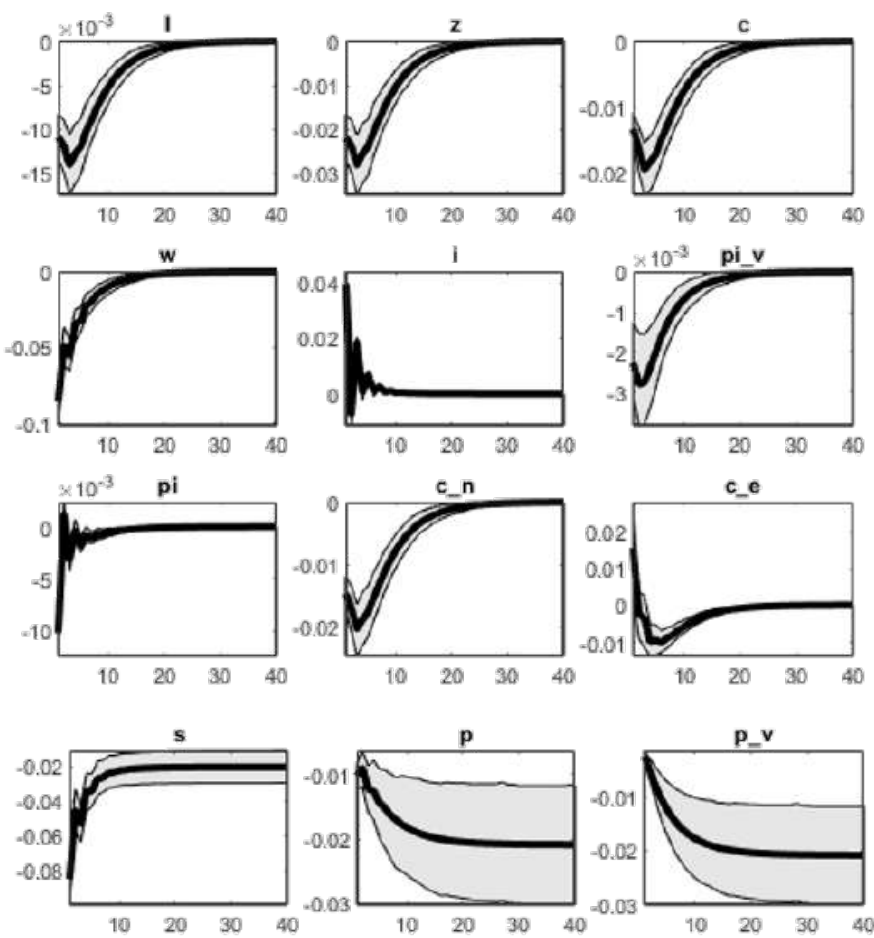

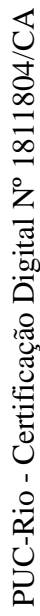
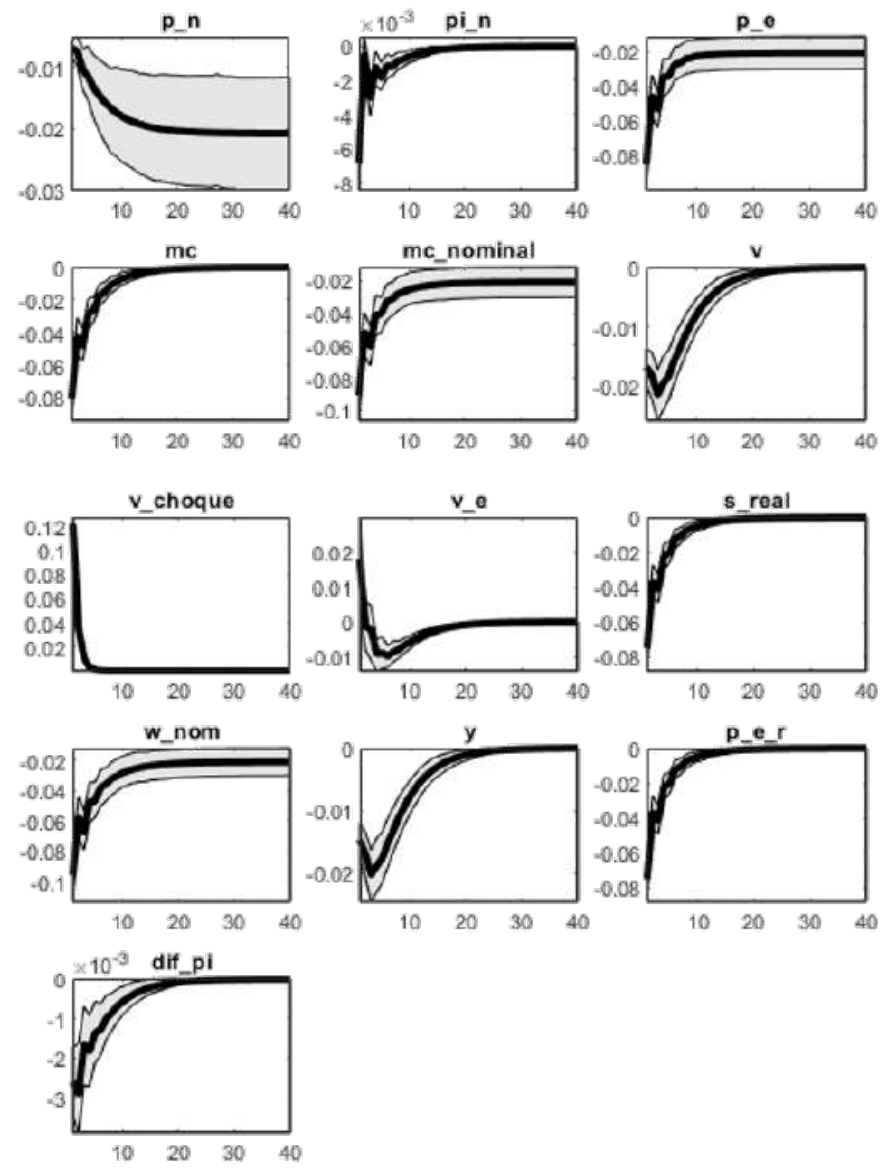
IRF: Choque nos preços internacionais de petróleo
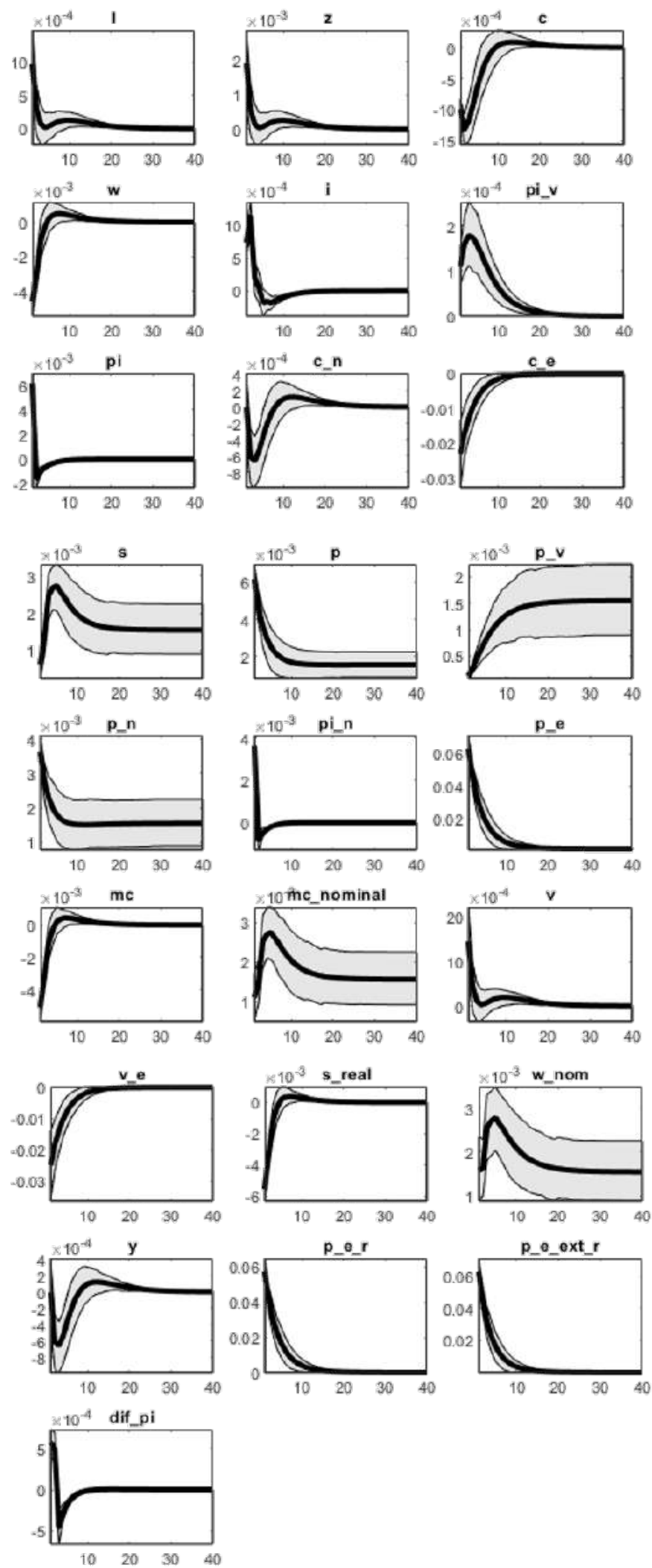
IRF: Choque nos preços de derivados nacionais
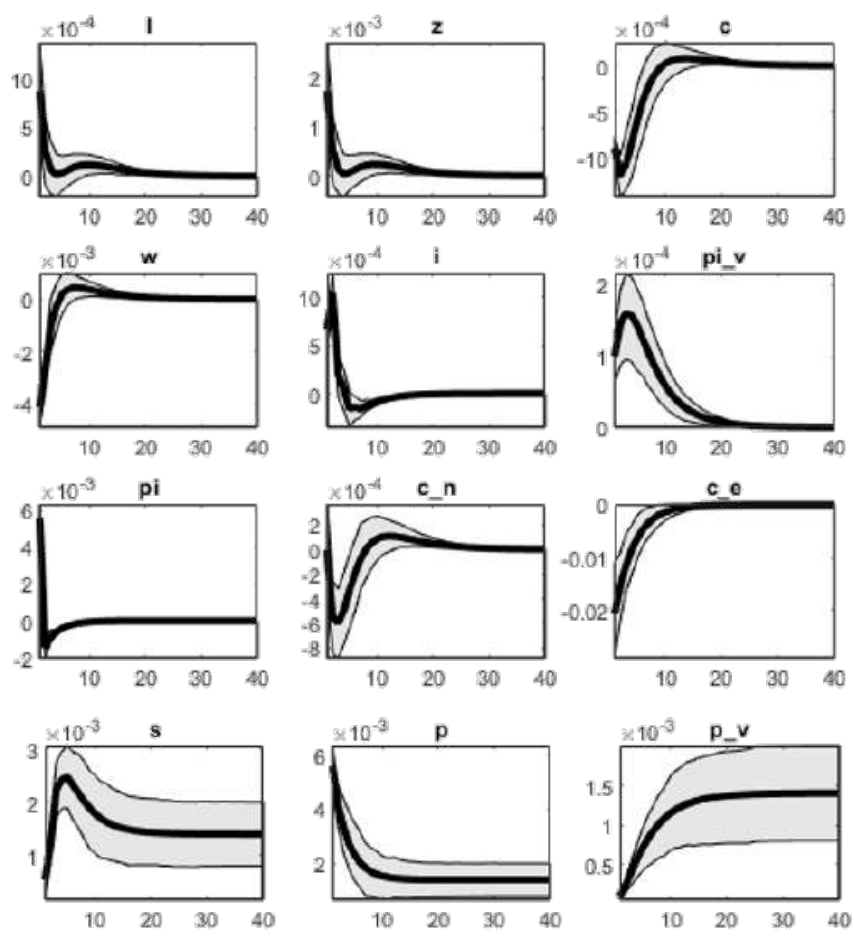

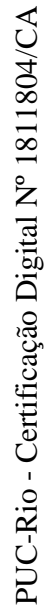
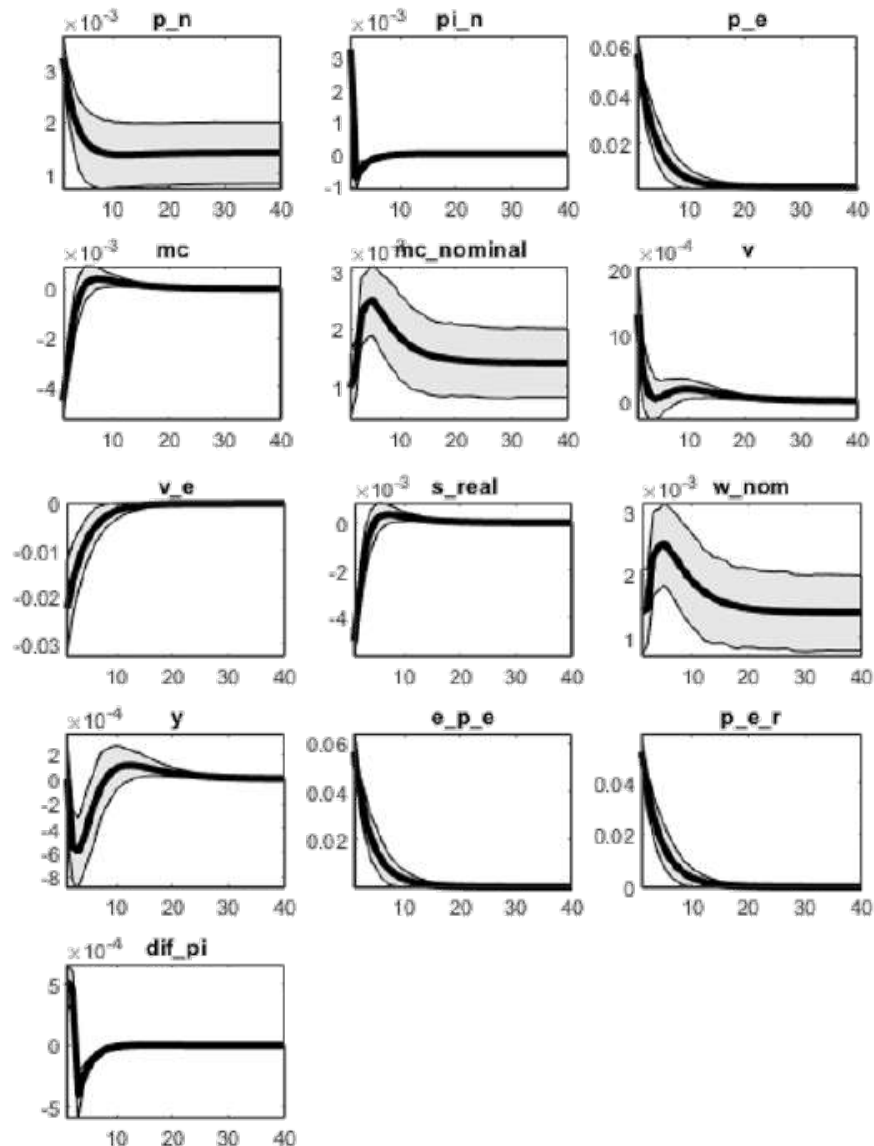
IRF: Choque de produtividade
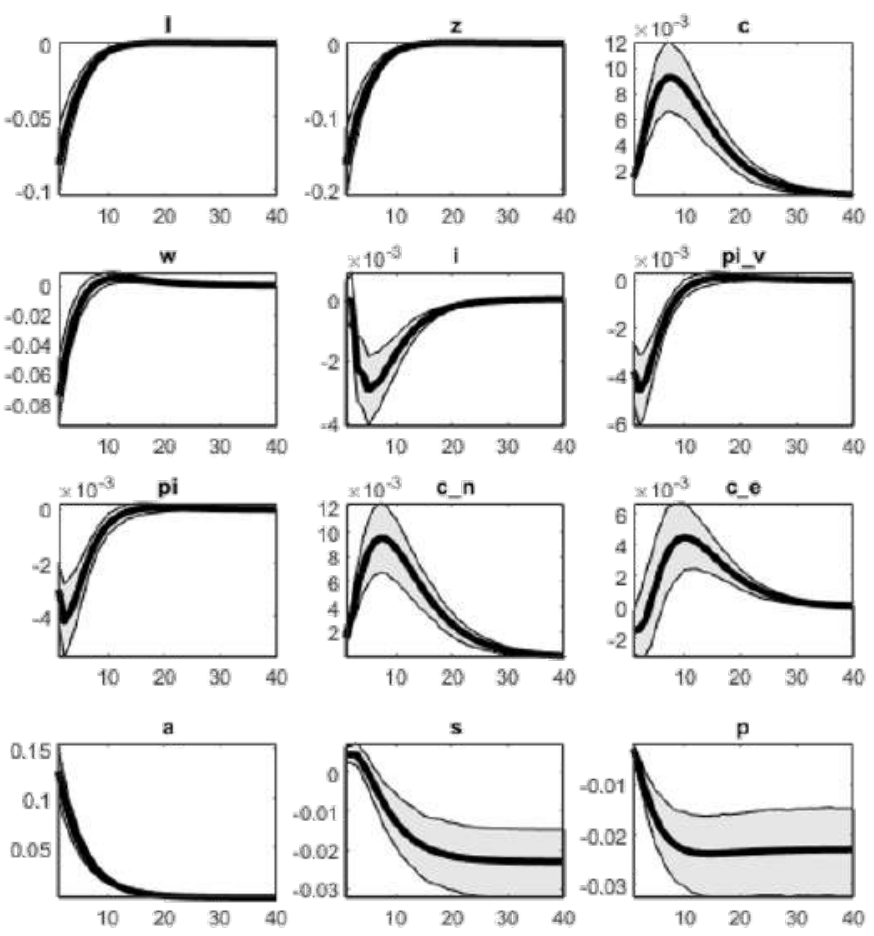

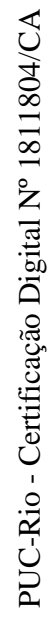
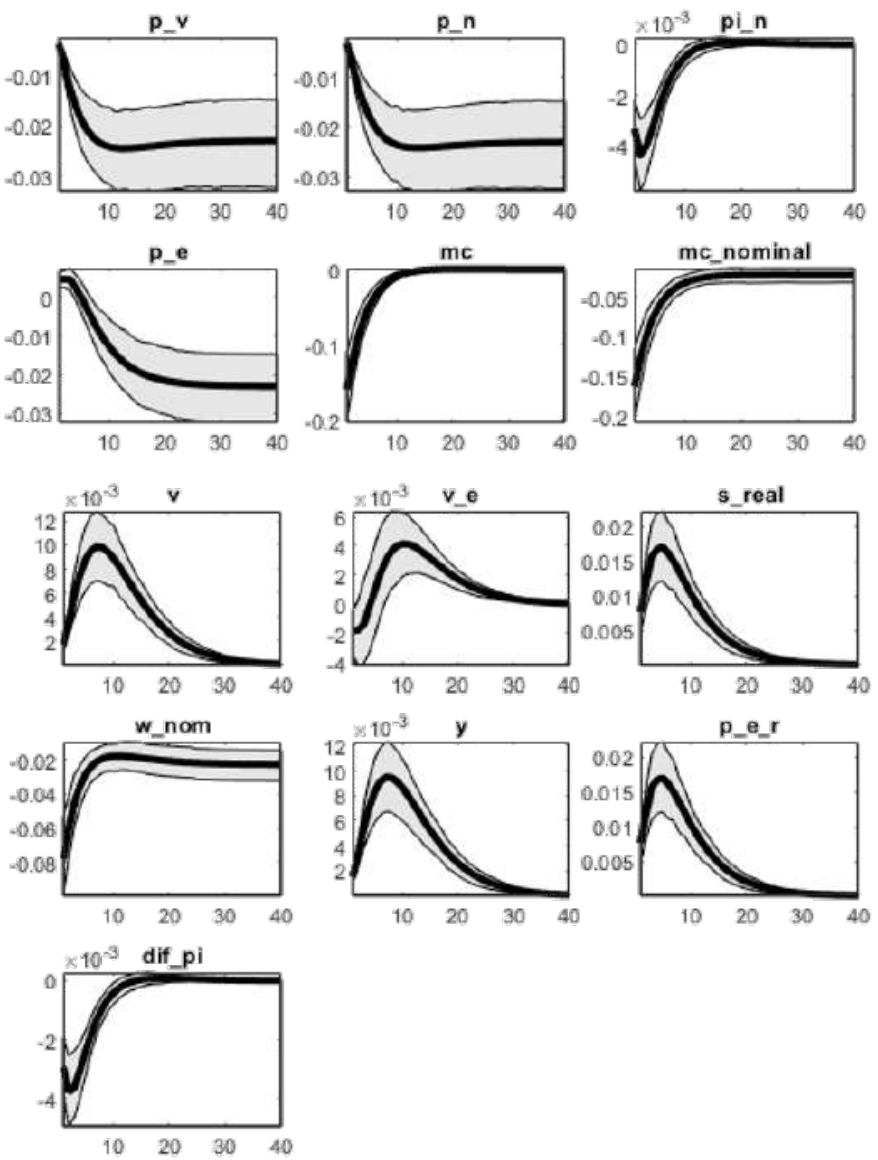
IRF: Choque de prêmio de risco
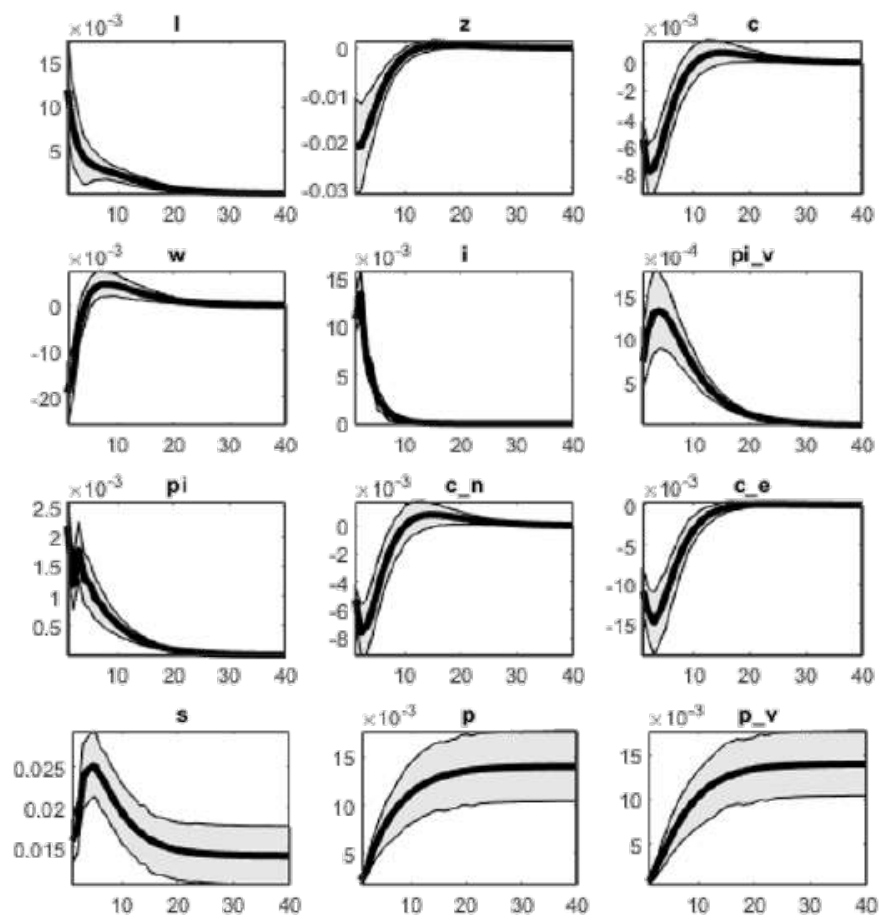

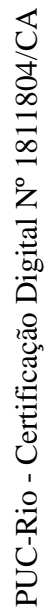
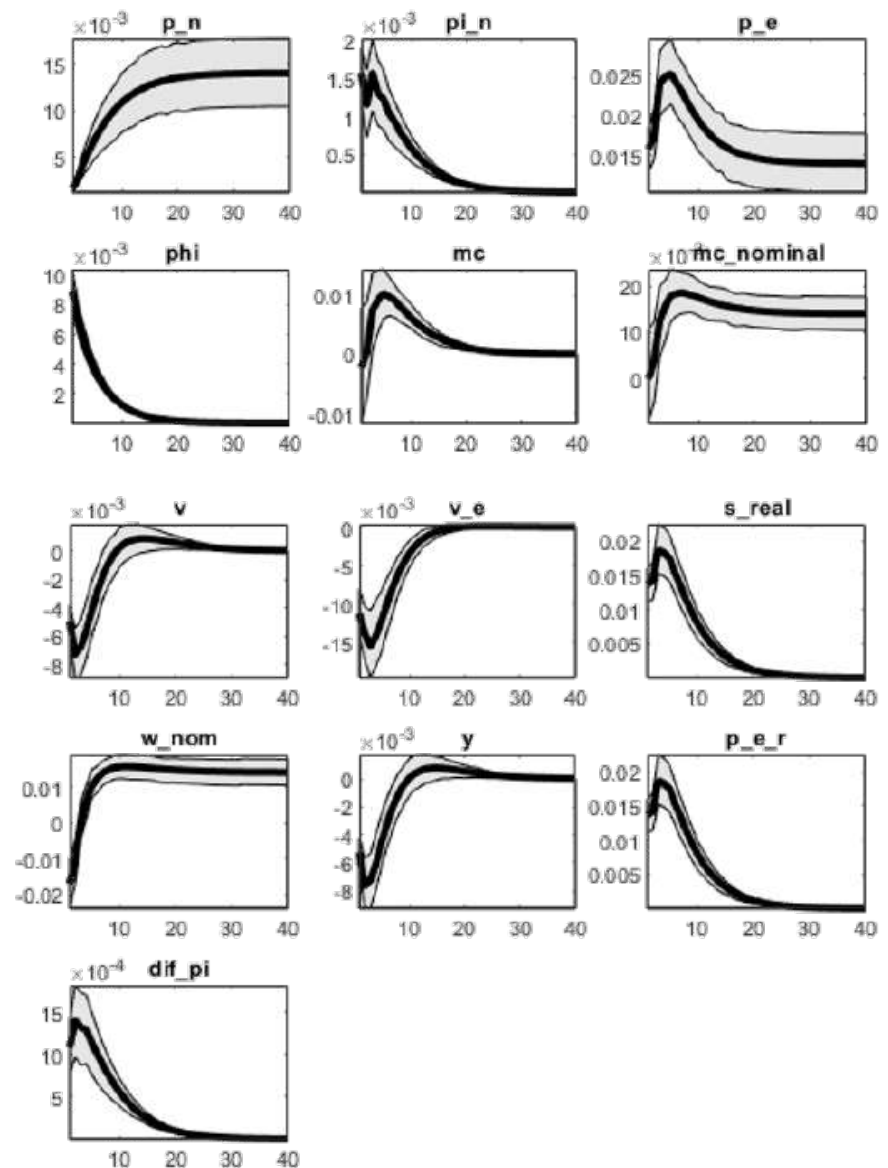
IRF: Choque na taxa de juros estrangeira
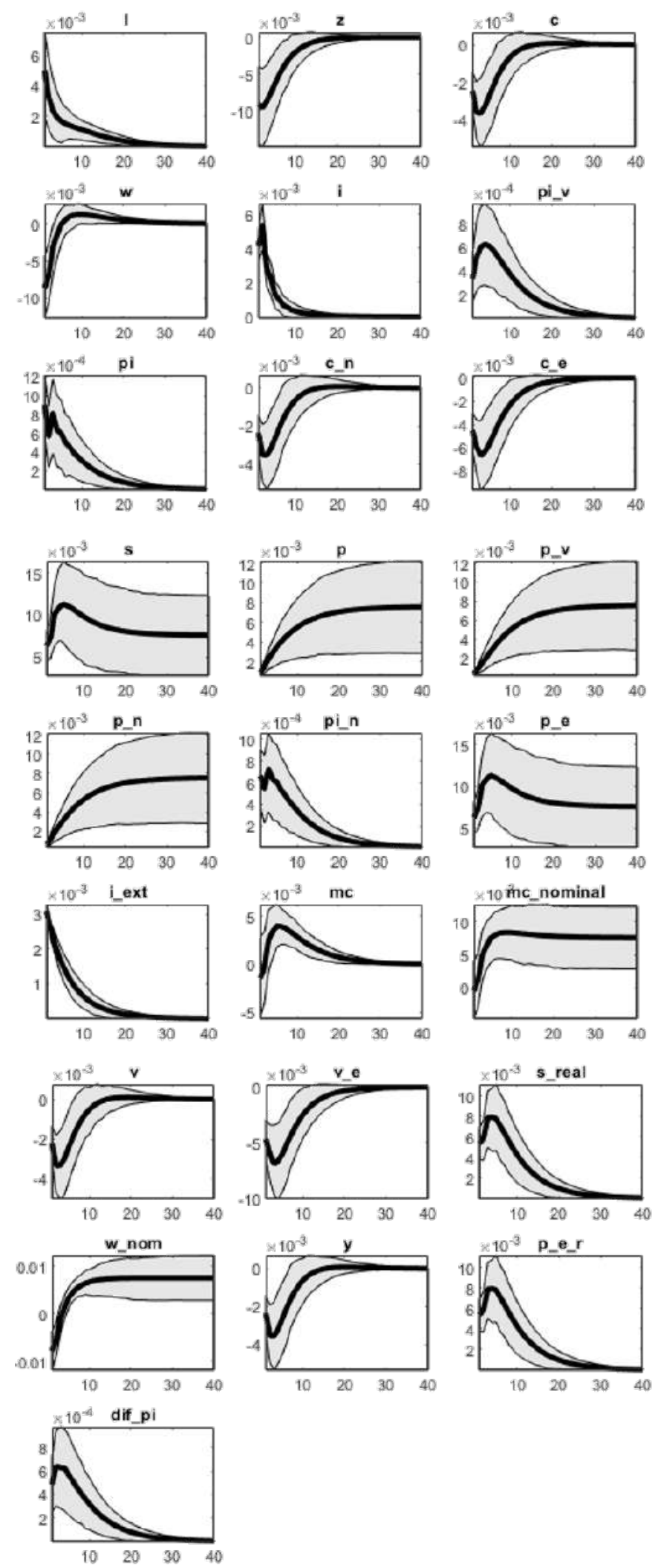


\subsection{2}

Alternativa III: Backward-looking e headline inflation

\section{MCMC diagnóstico multivariado}
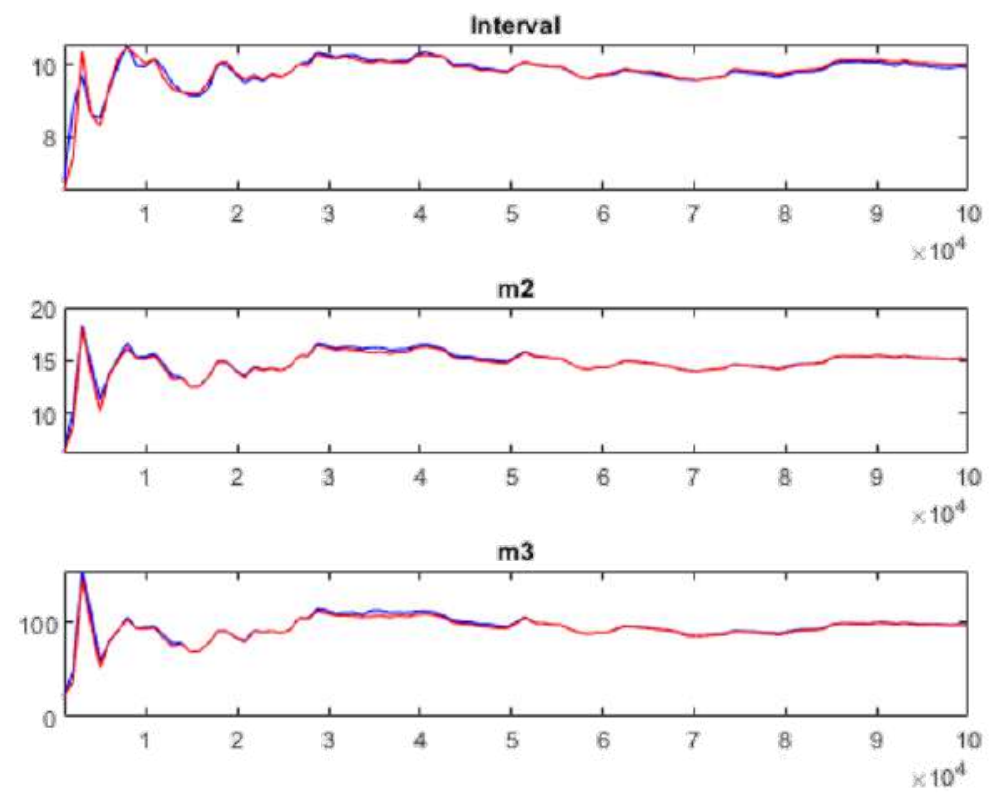

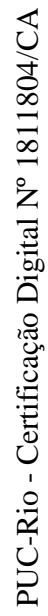

Historical x smoothed variables
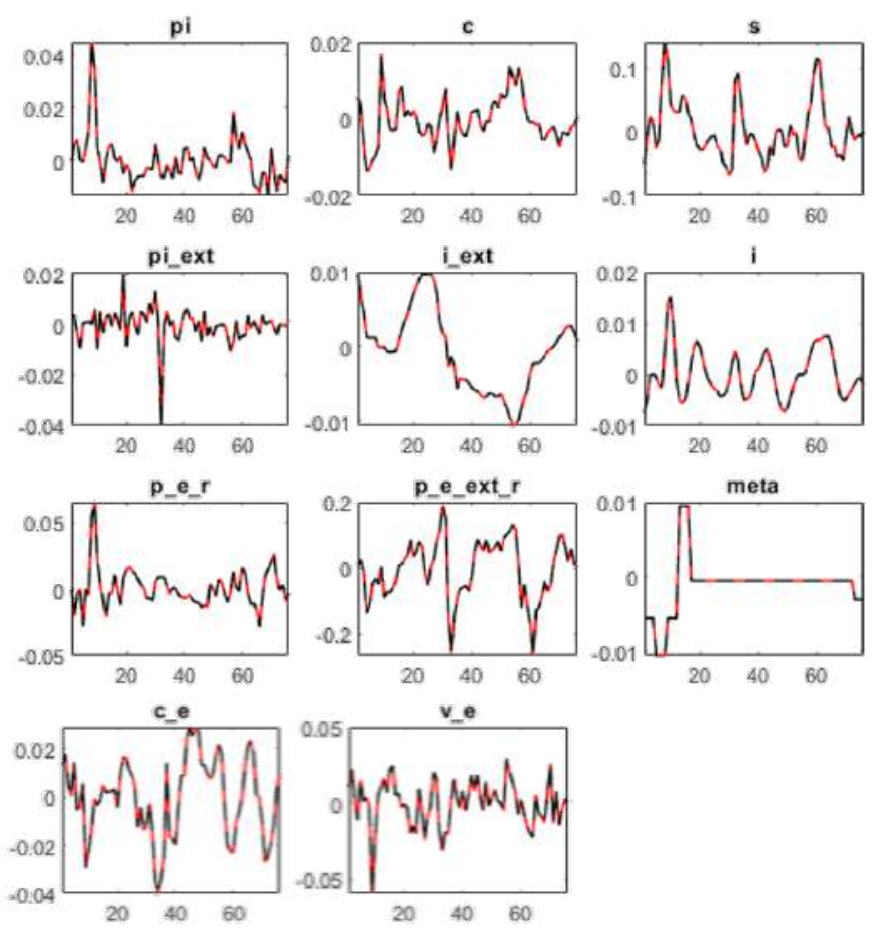
Smoothed shocks
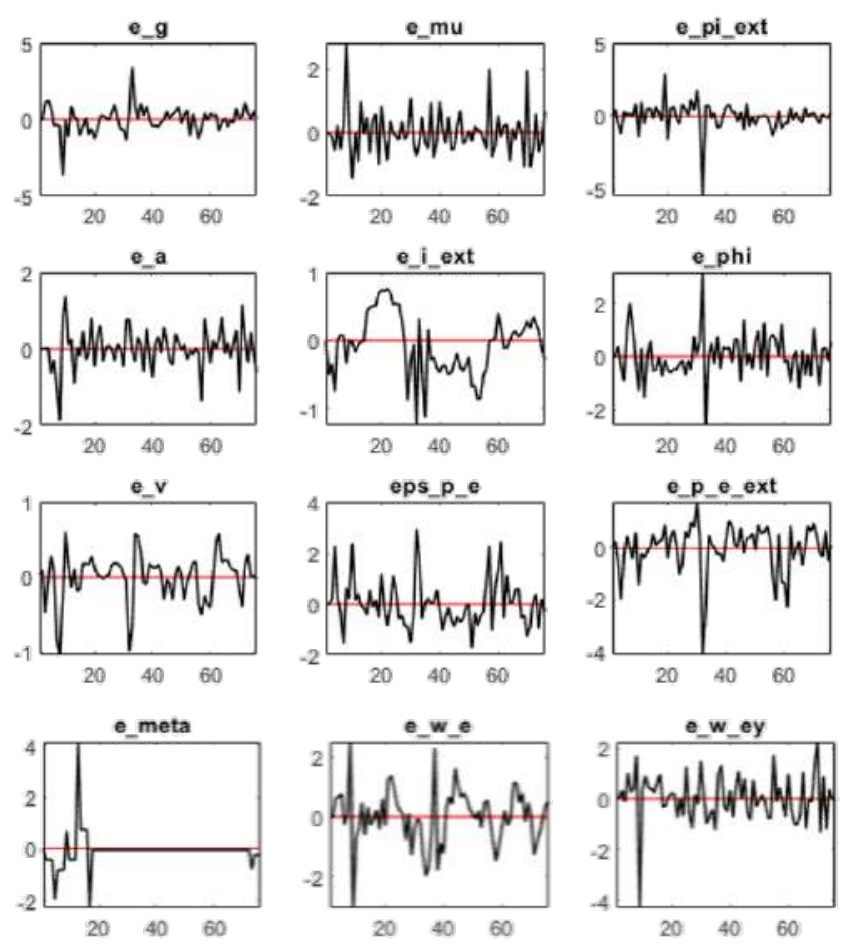

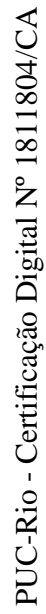

Priors x posteriors
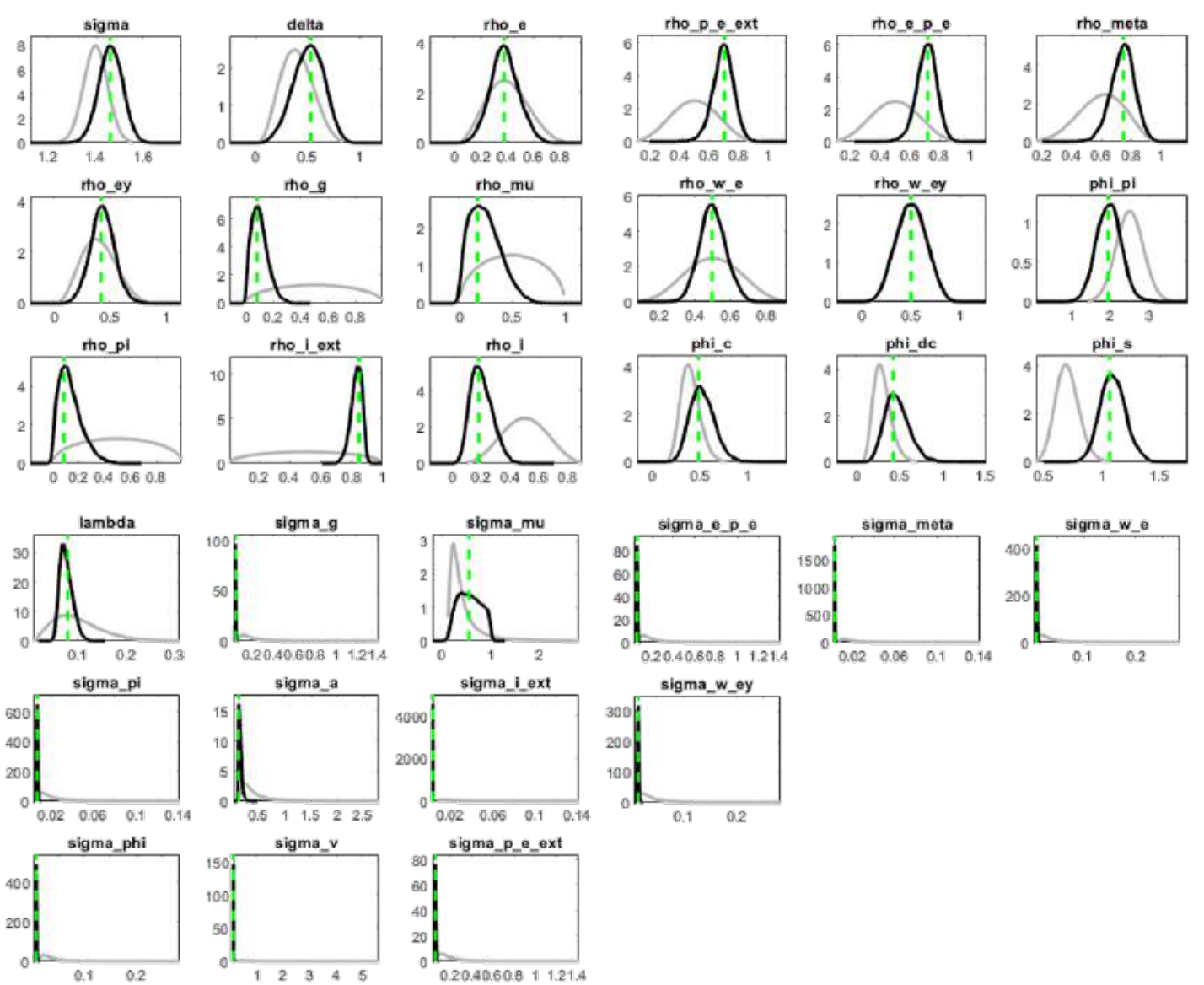
IRF: Choque de política monetária
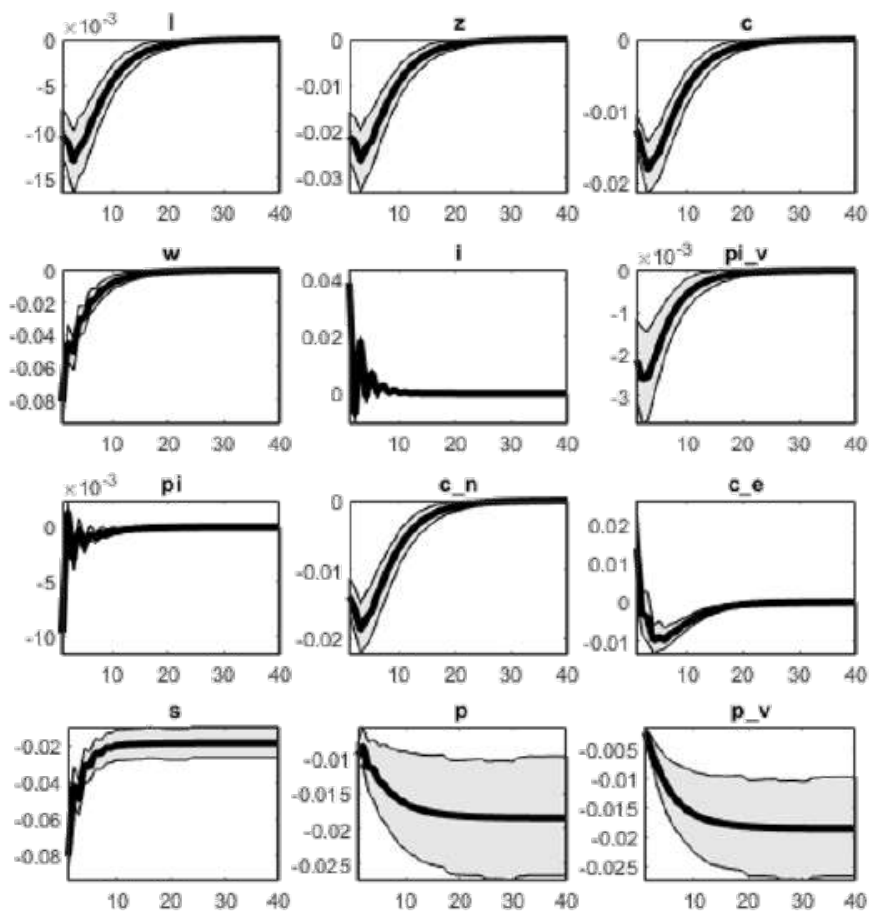

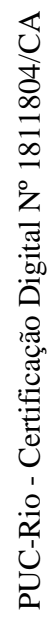
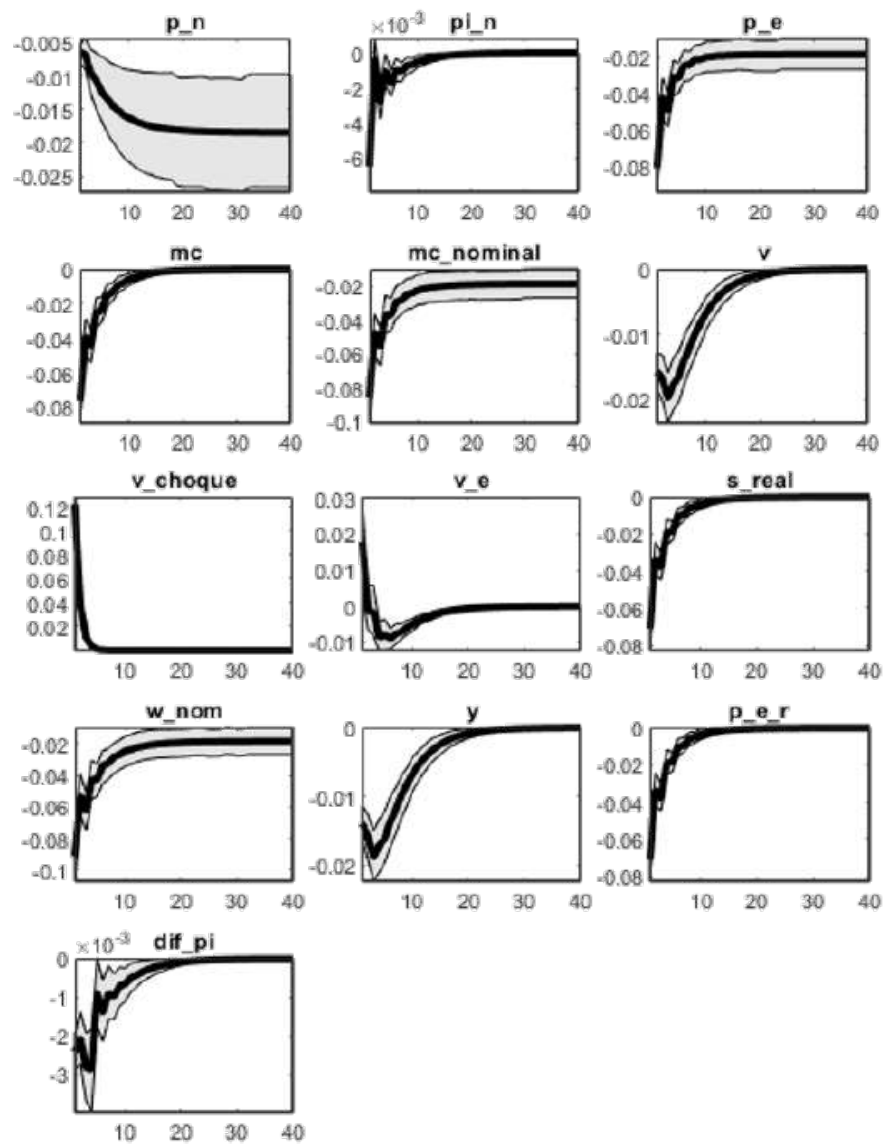
IRF: Choque nos preços internacionais de petróleo
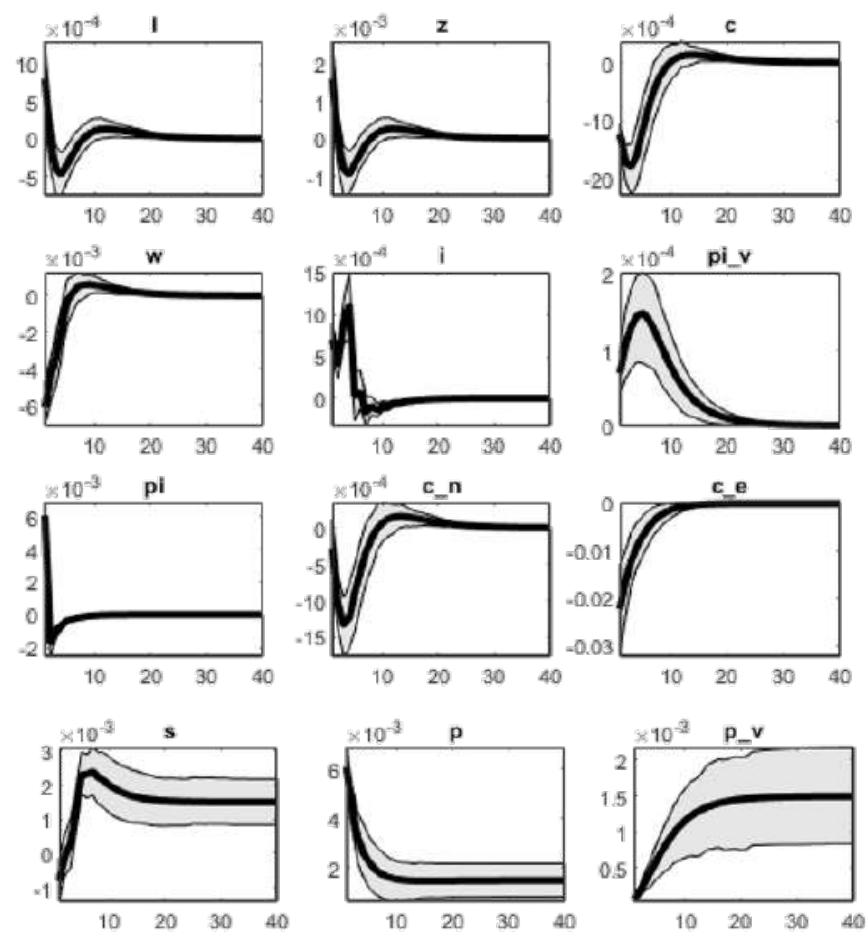

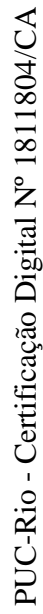
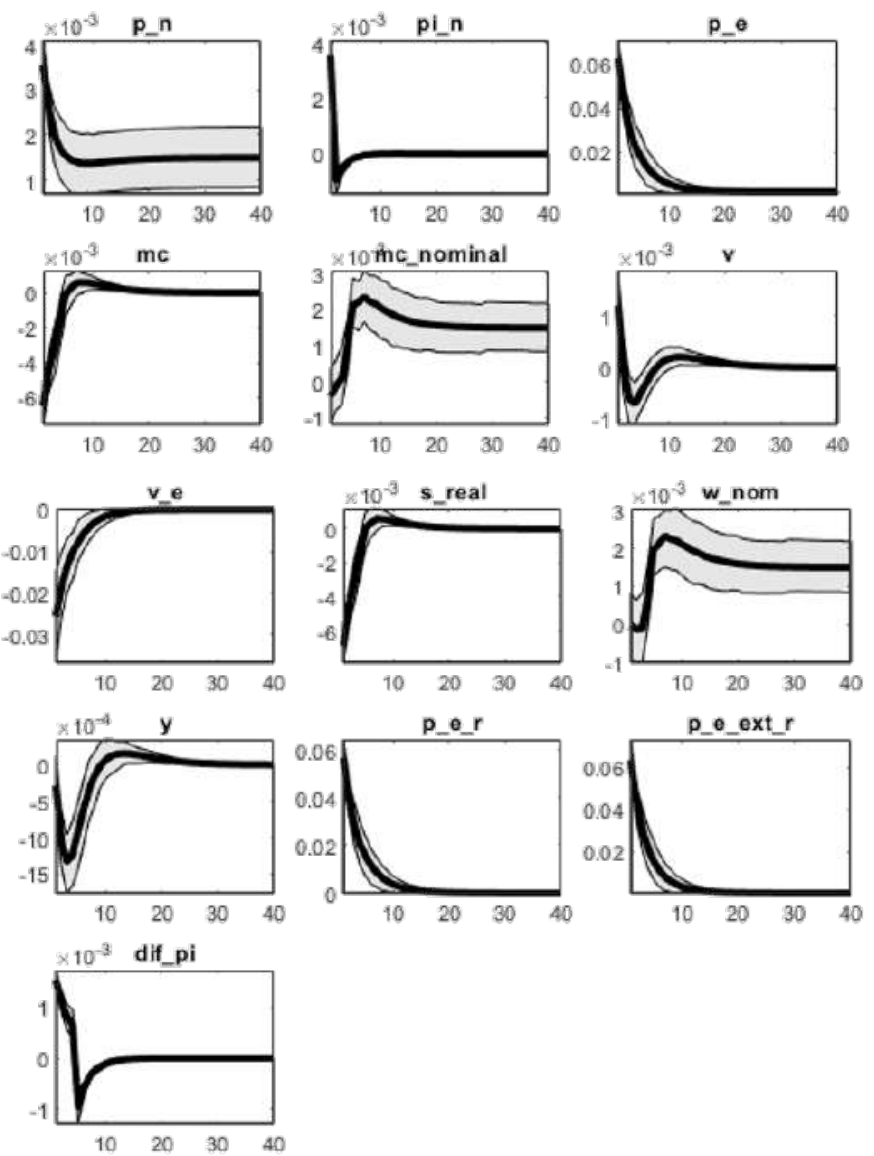
IRF: Choque nos preços de derivados nacionais
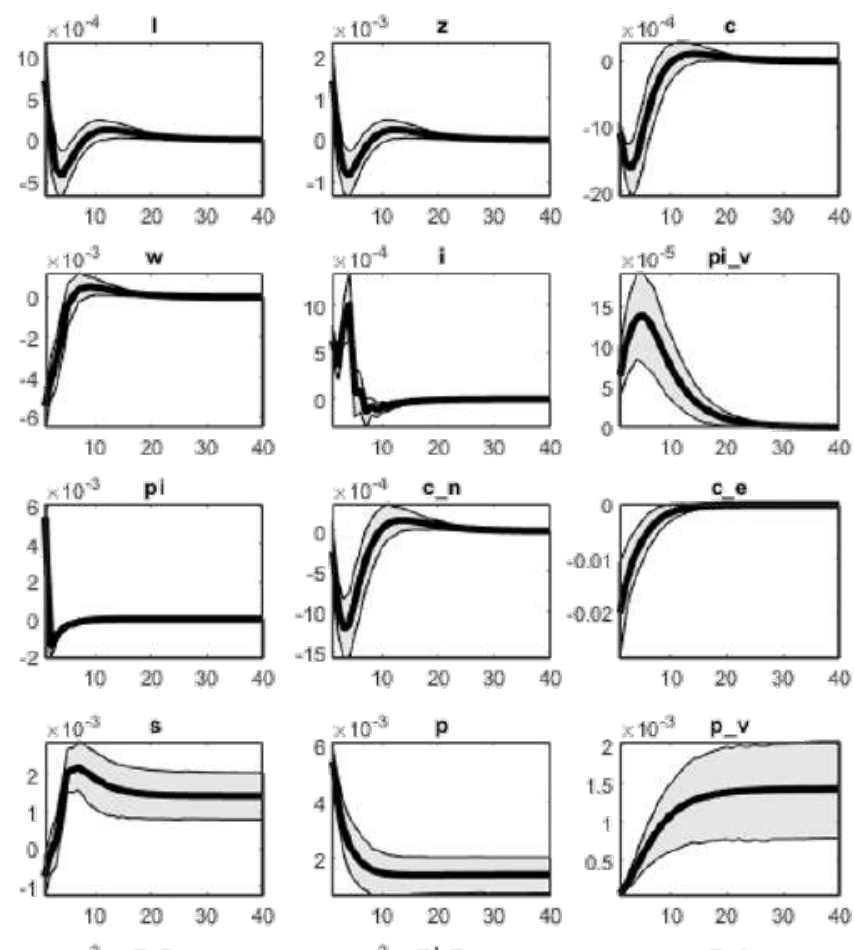

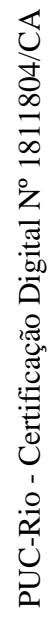
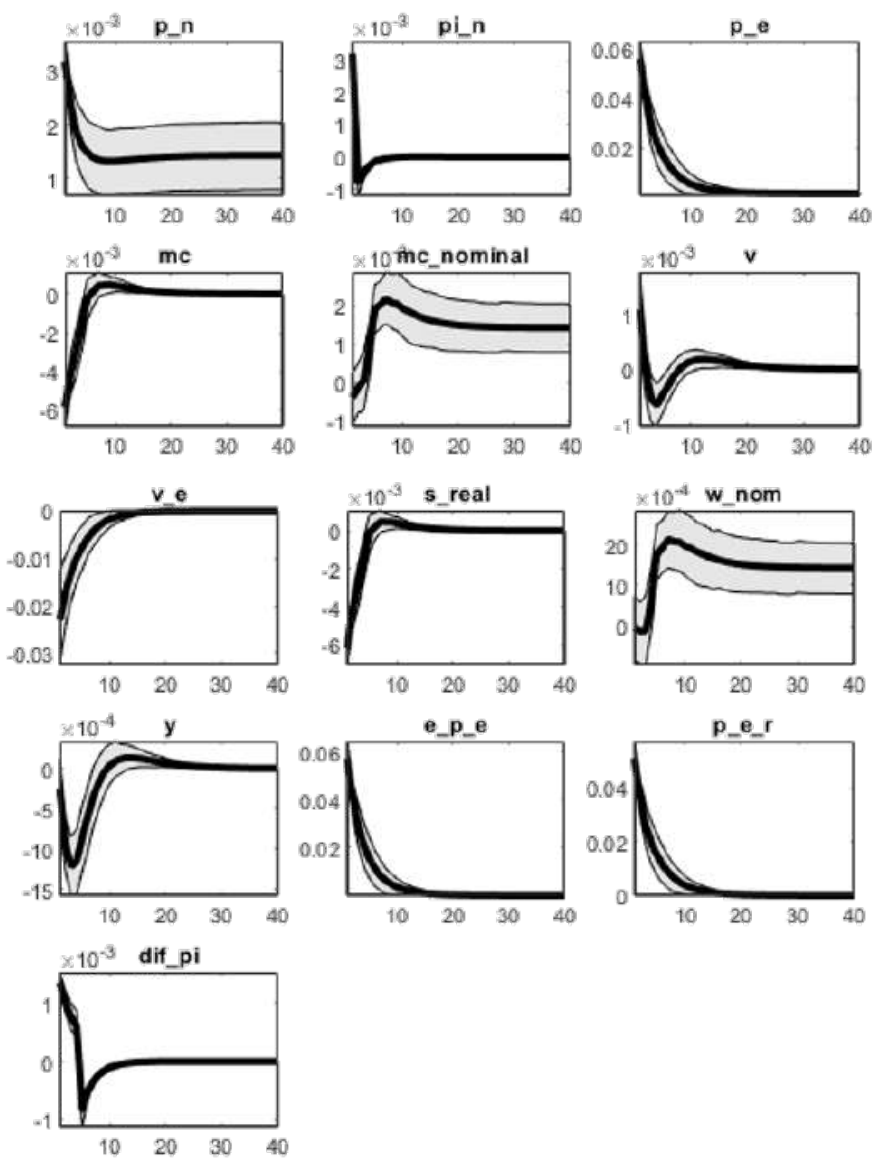
IRF: Choque de produtividade
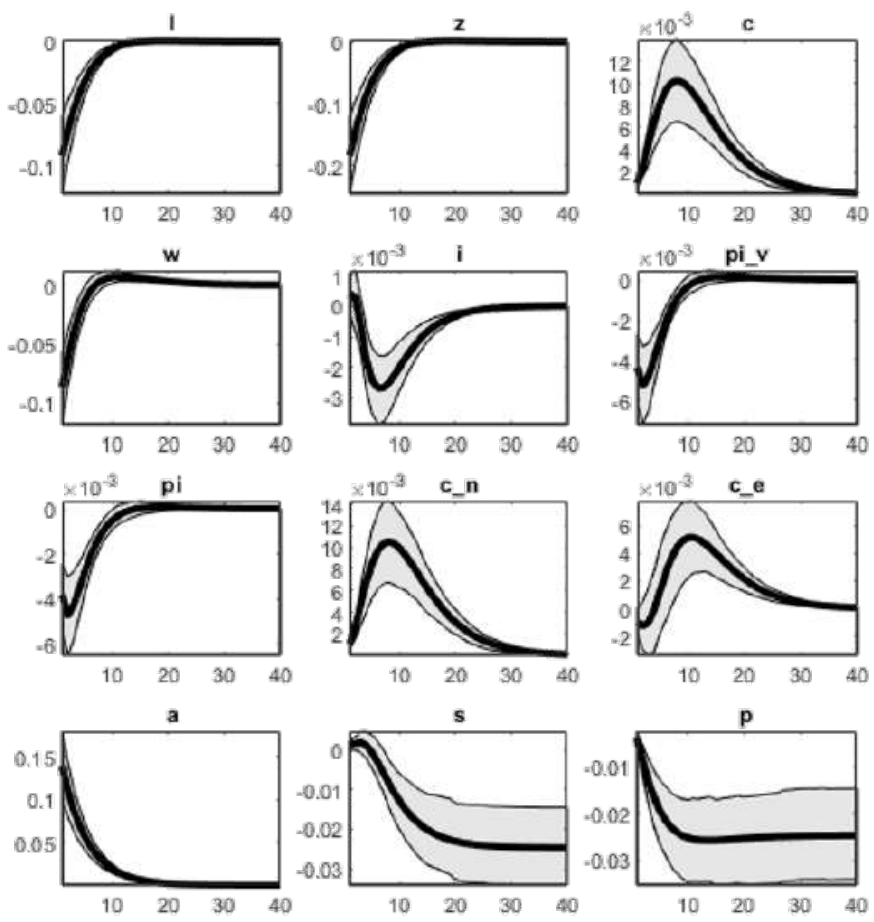

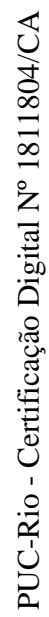
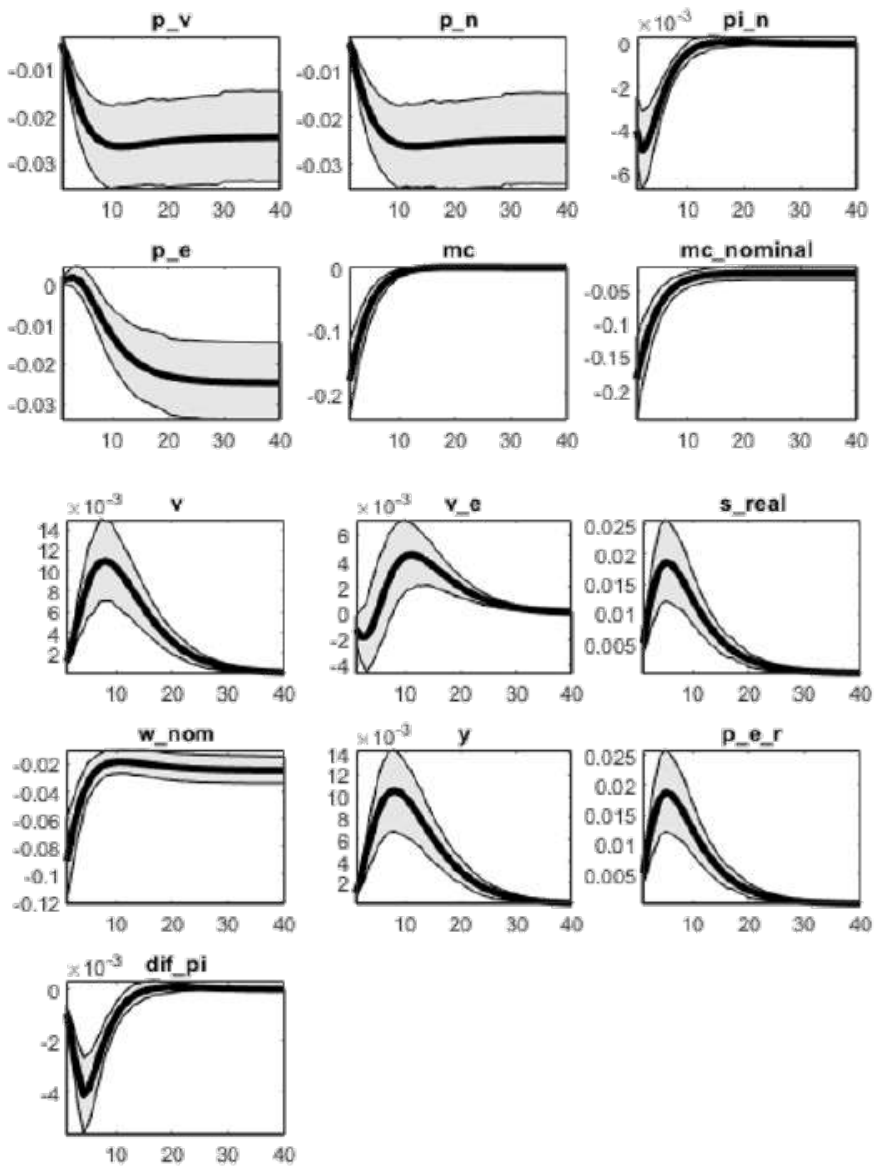
IRF: Choque de prêmio de risco
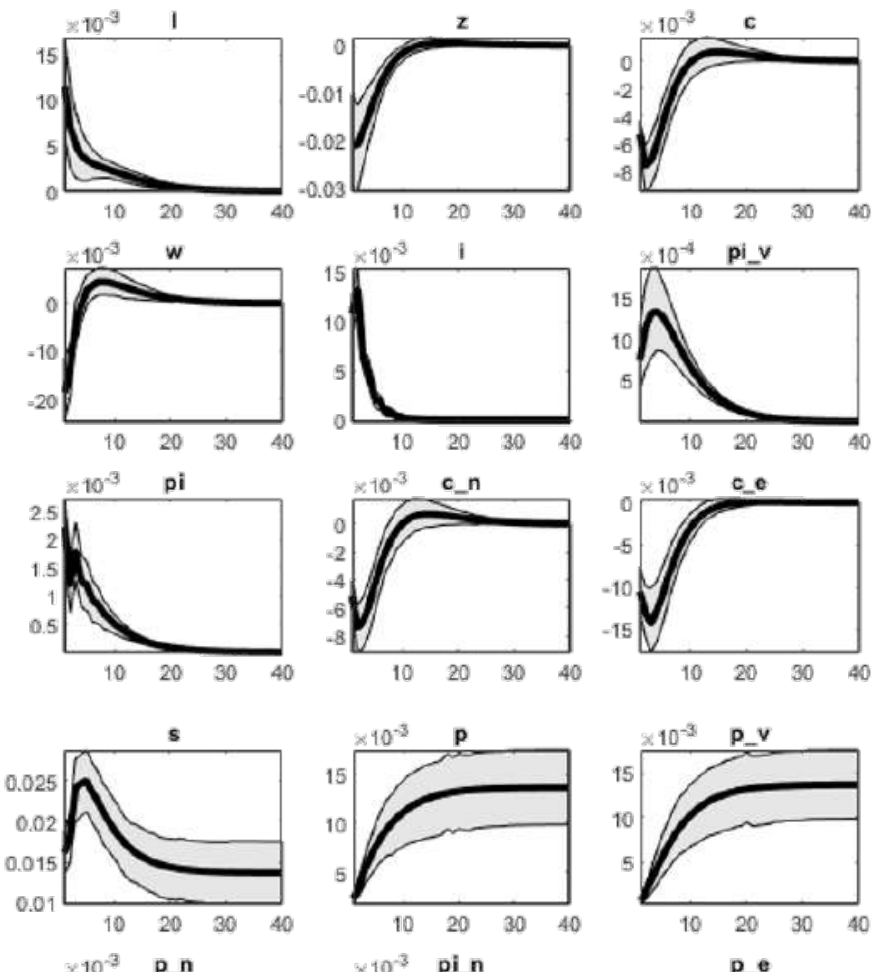

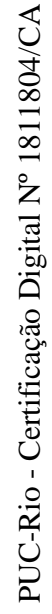
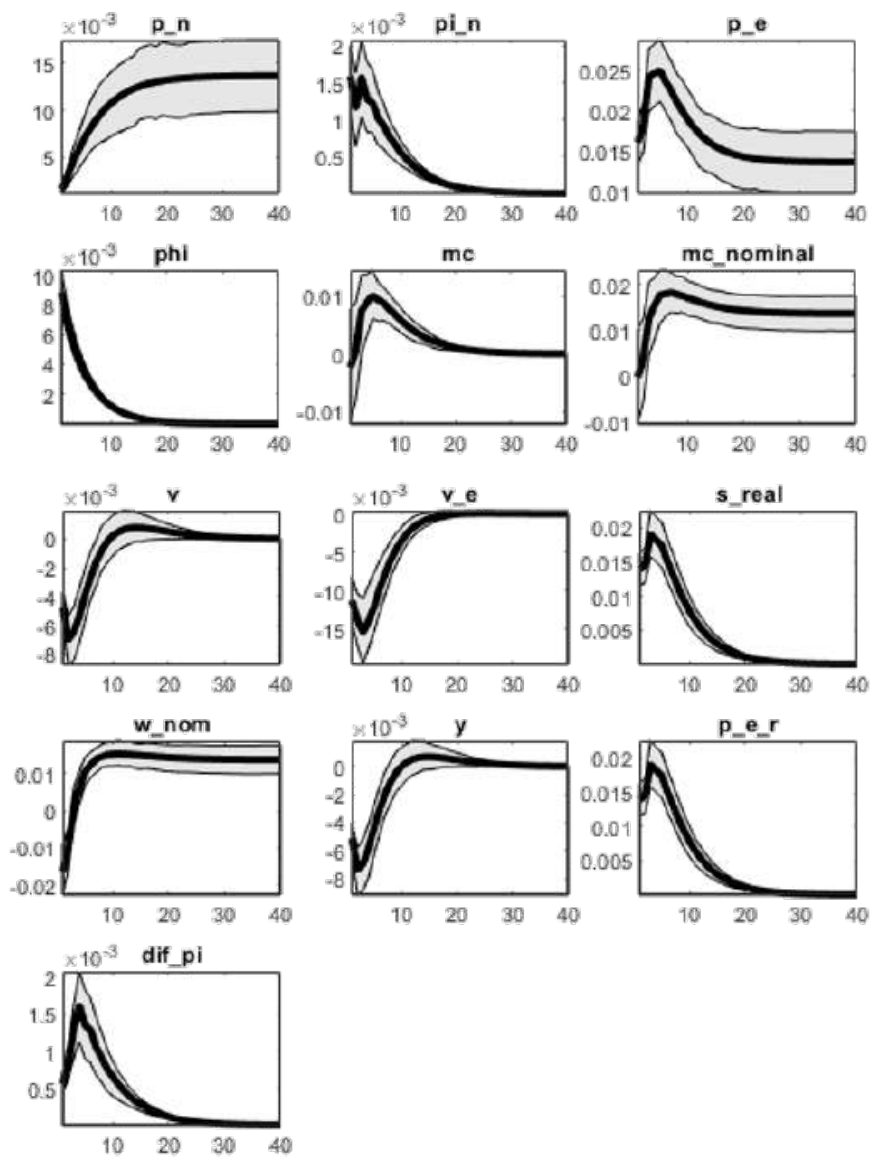
IRF: Choque na taxa de juros estrangeira
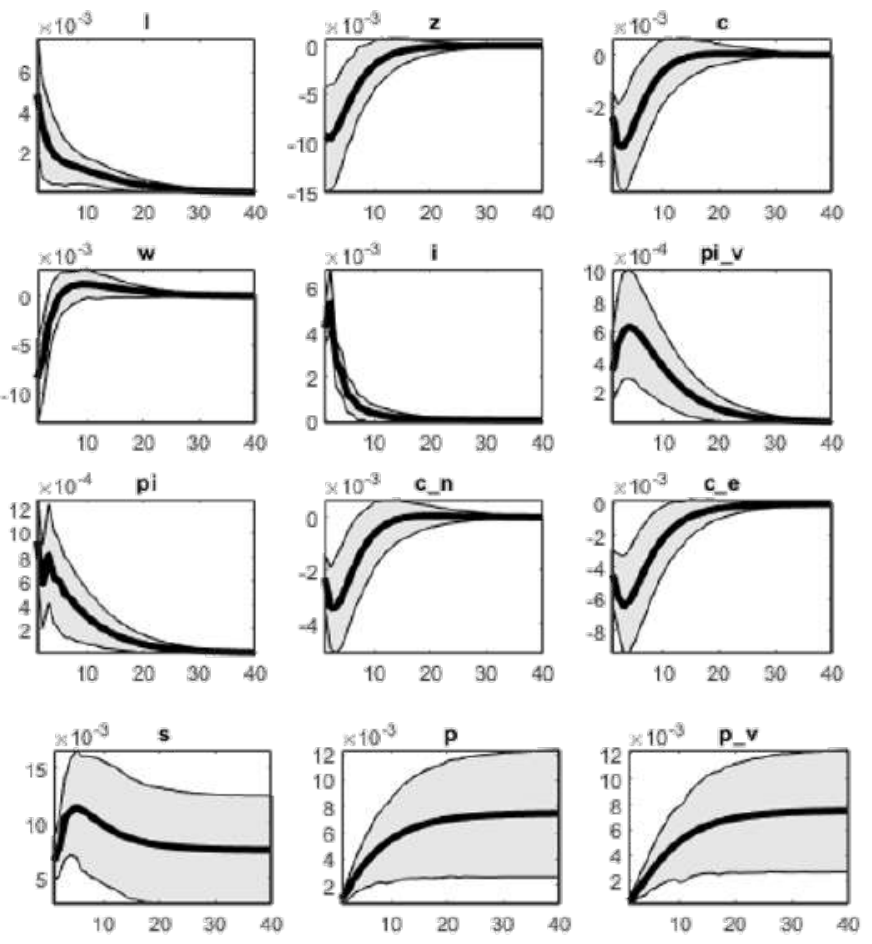

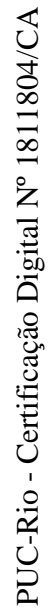
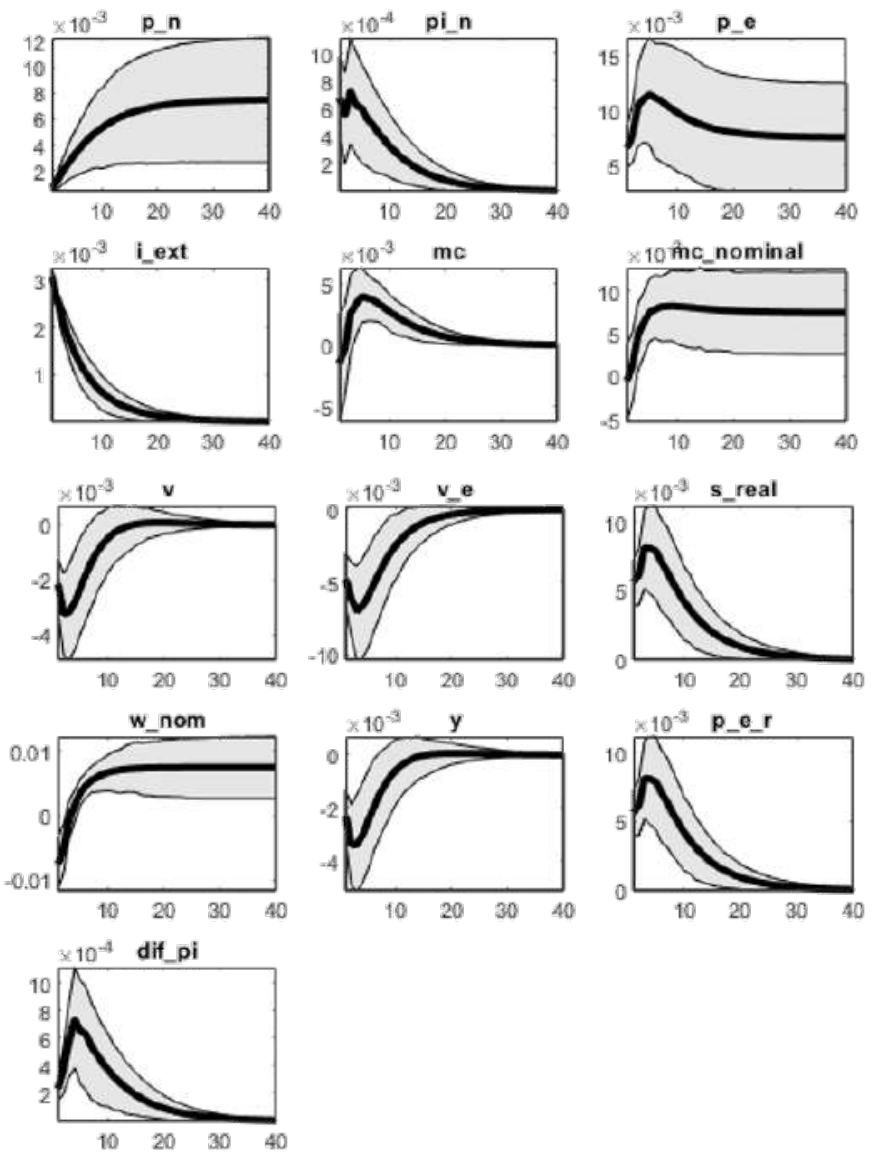


\subsection{3}

Alternativa IV: Backward-looking e core inflation

Backward-looking e core inflation:

MCMC diagnóstico multivariado
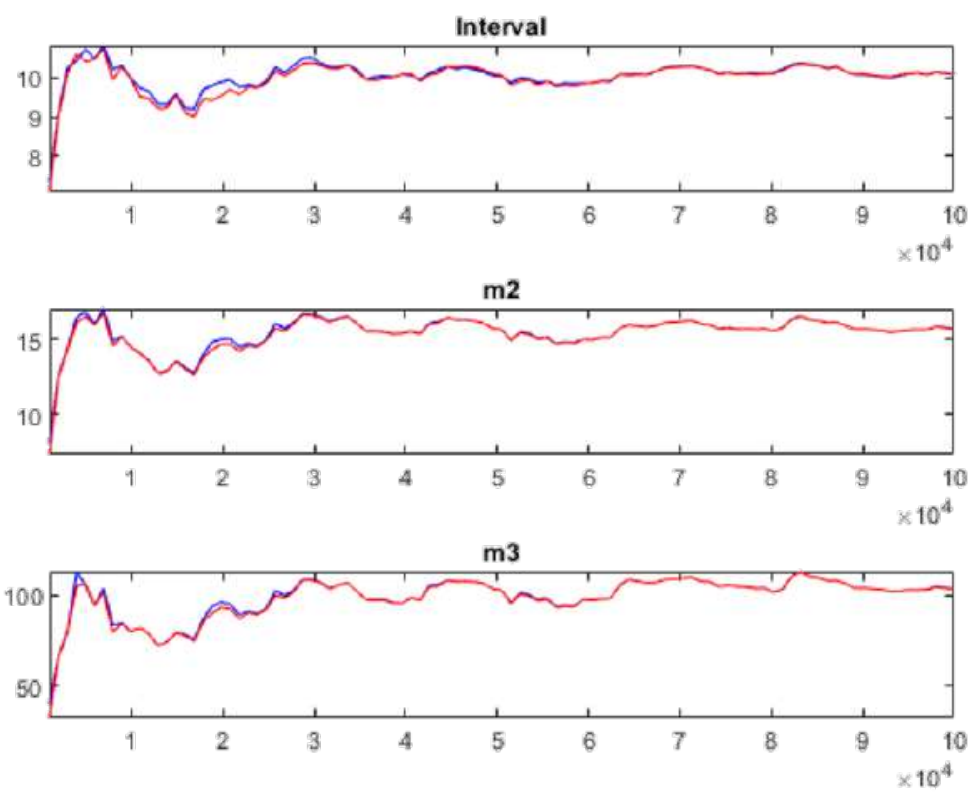

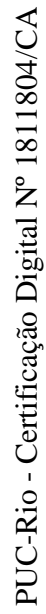

Historical x smoothed variables
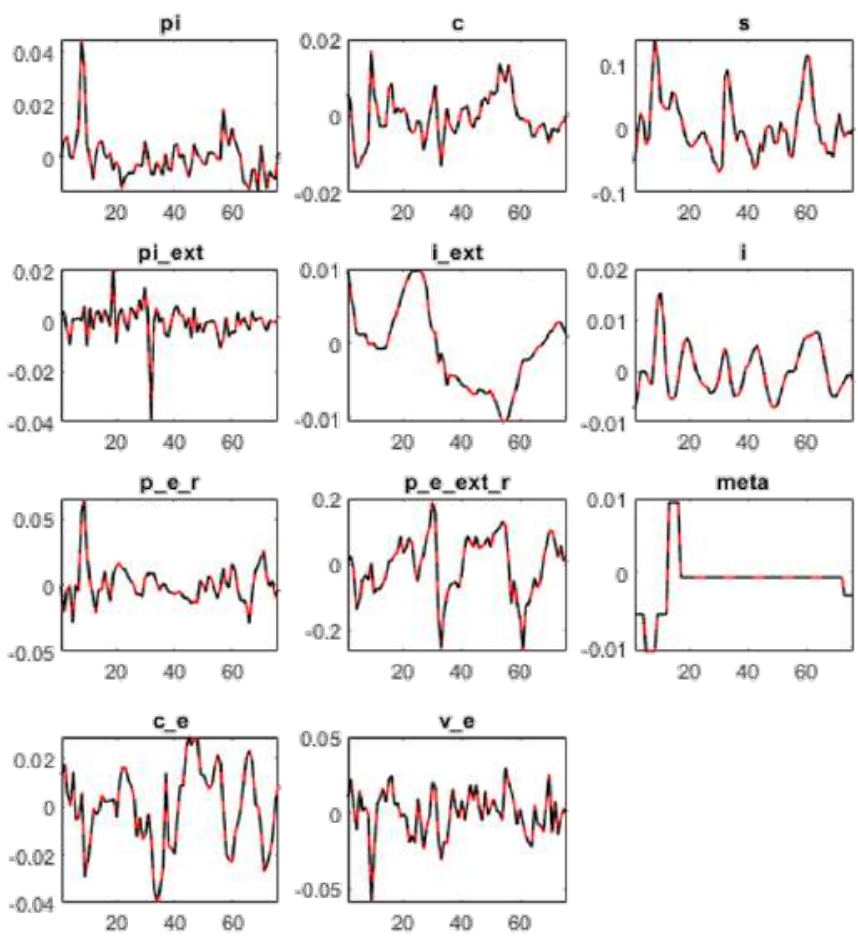
Smoothed shocks
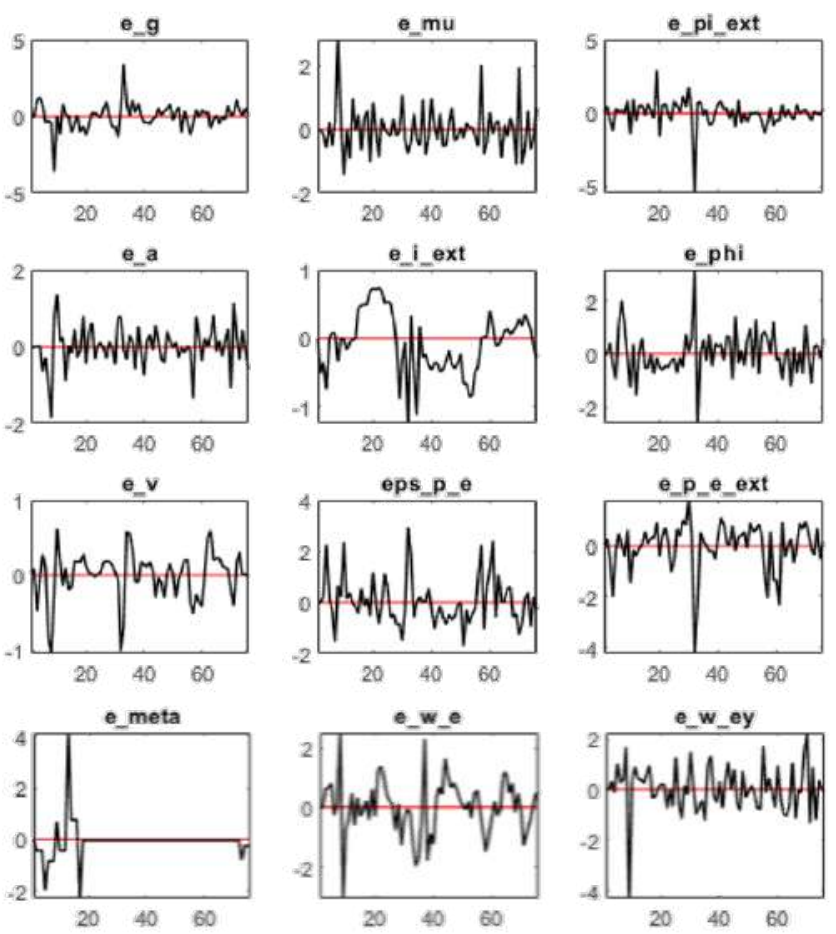

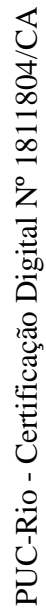

Backward-looking e core inflation: Priors x posteriors
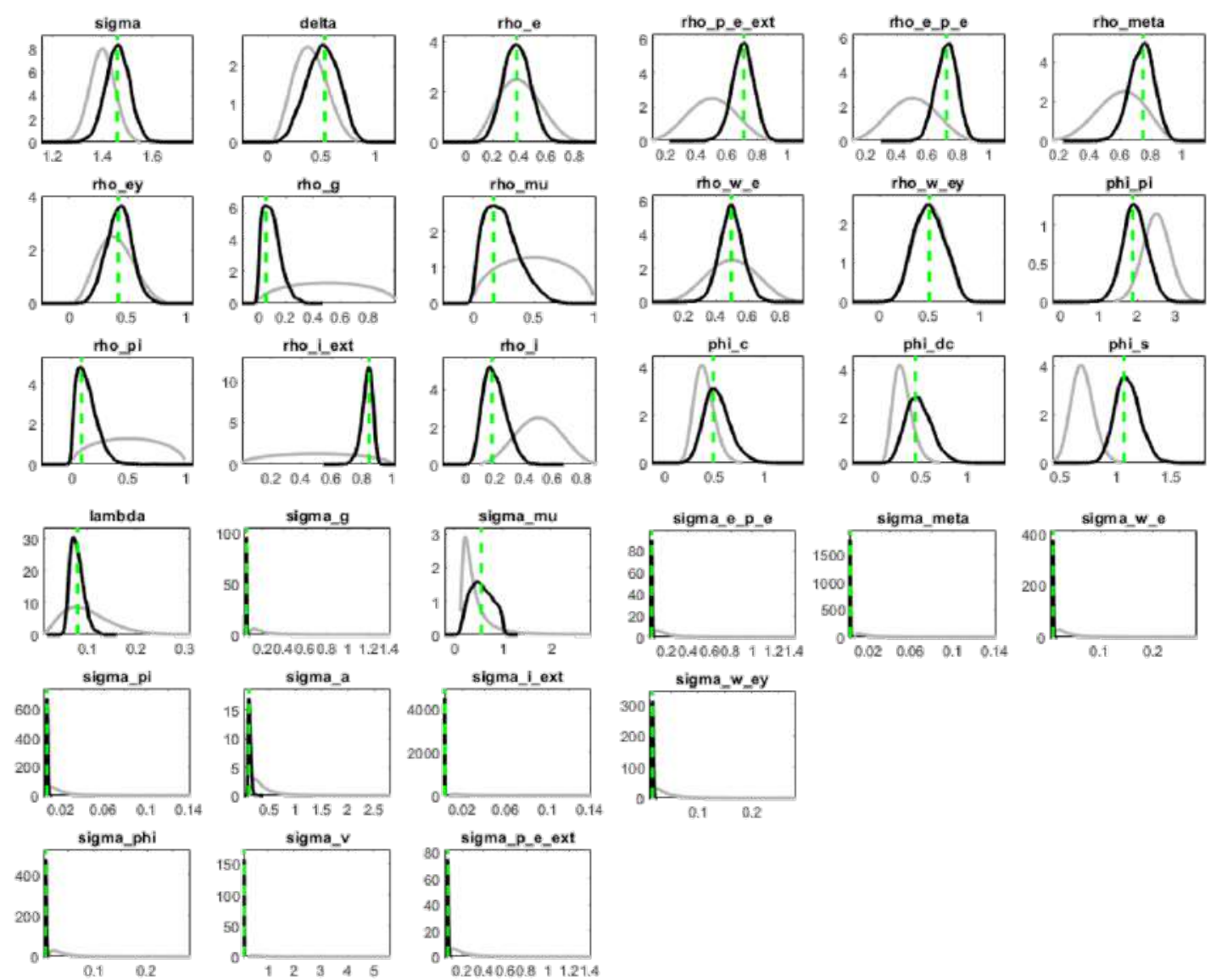
IRF: Choque de política monetária
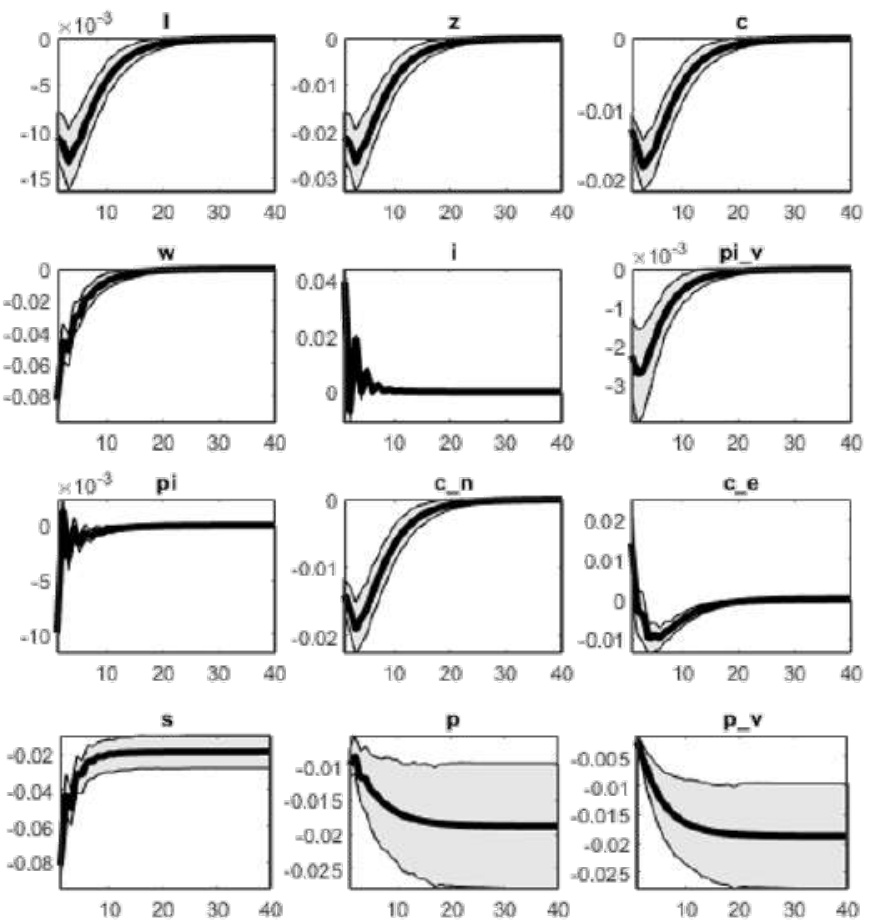

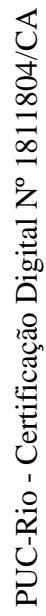
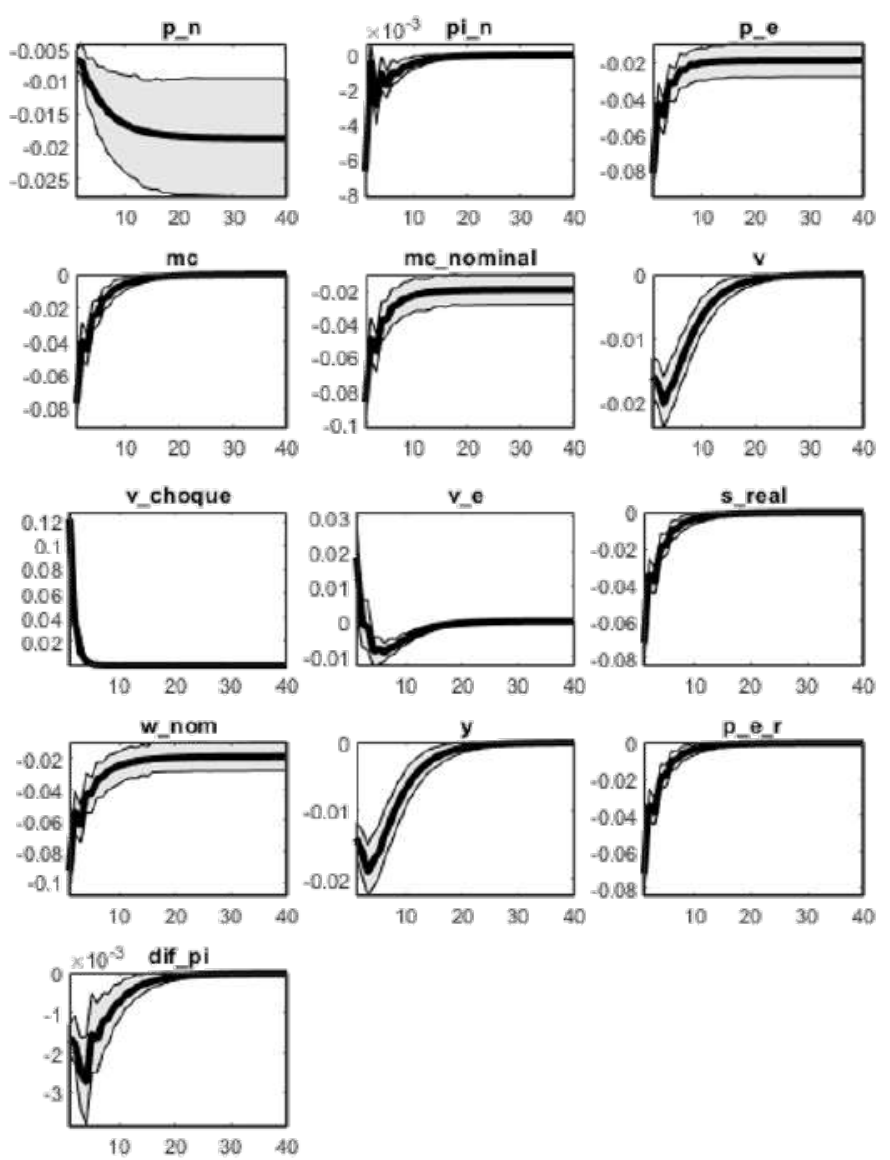
IRF: Choque nos preços internacionais de petróleo
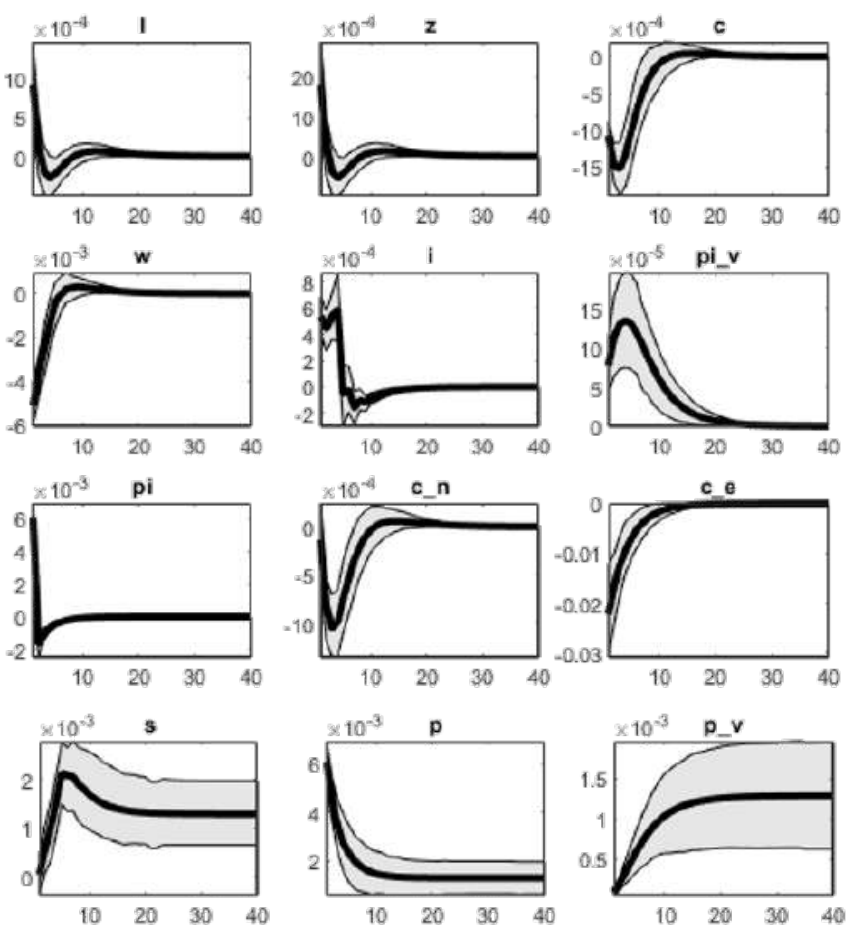

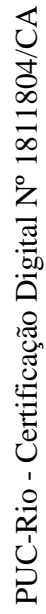
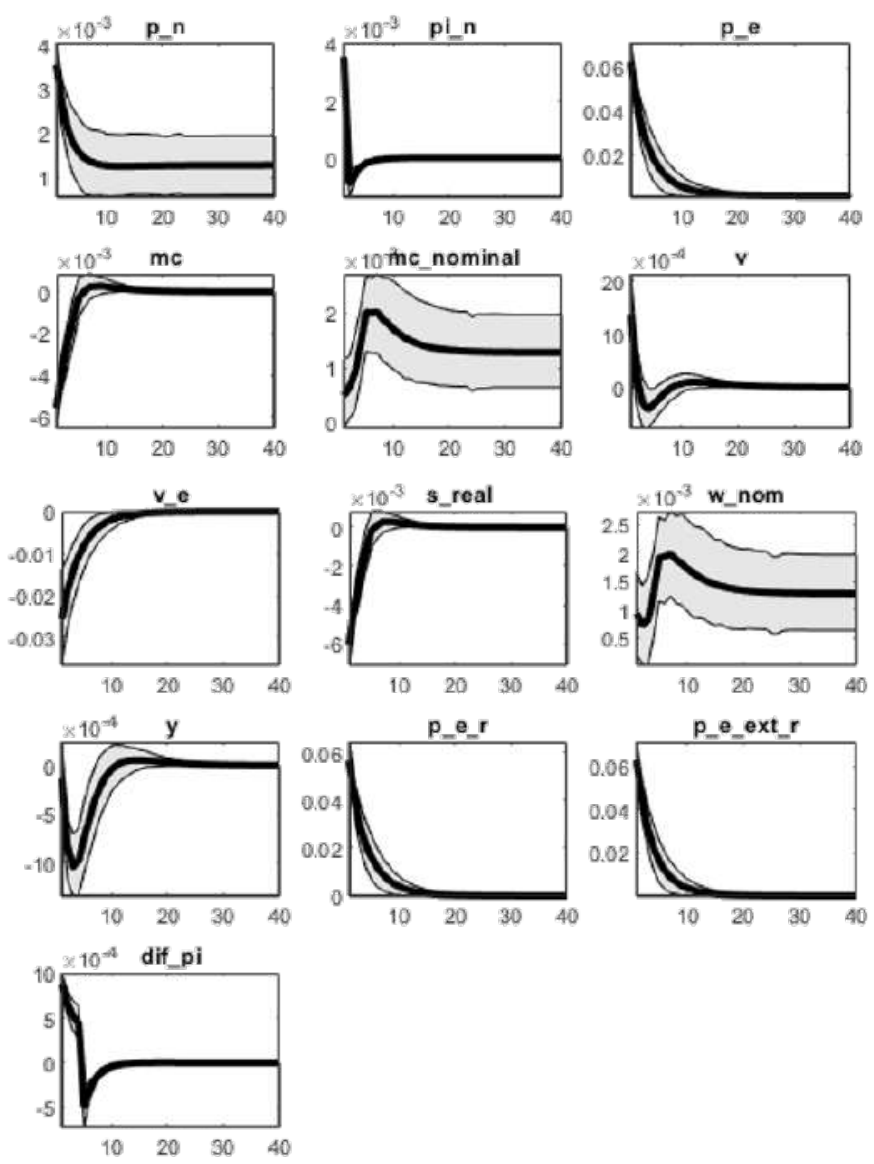
IRF: Choque nos preços de derivados nacionais
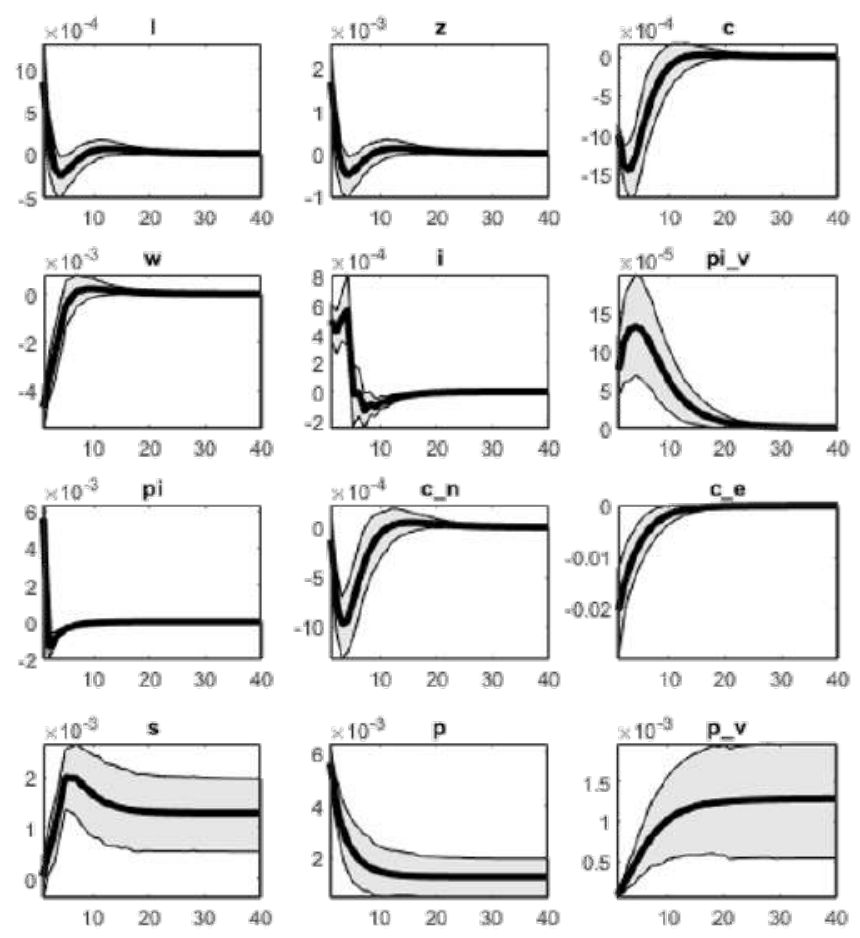

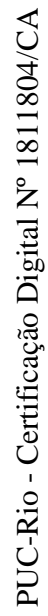
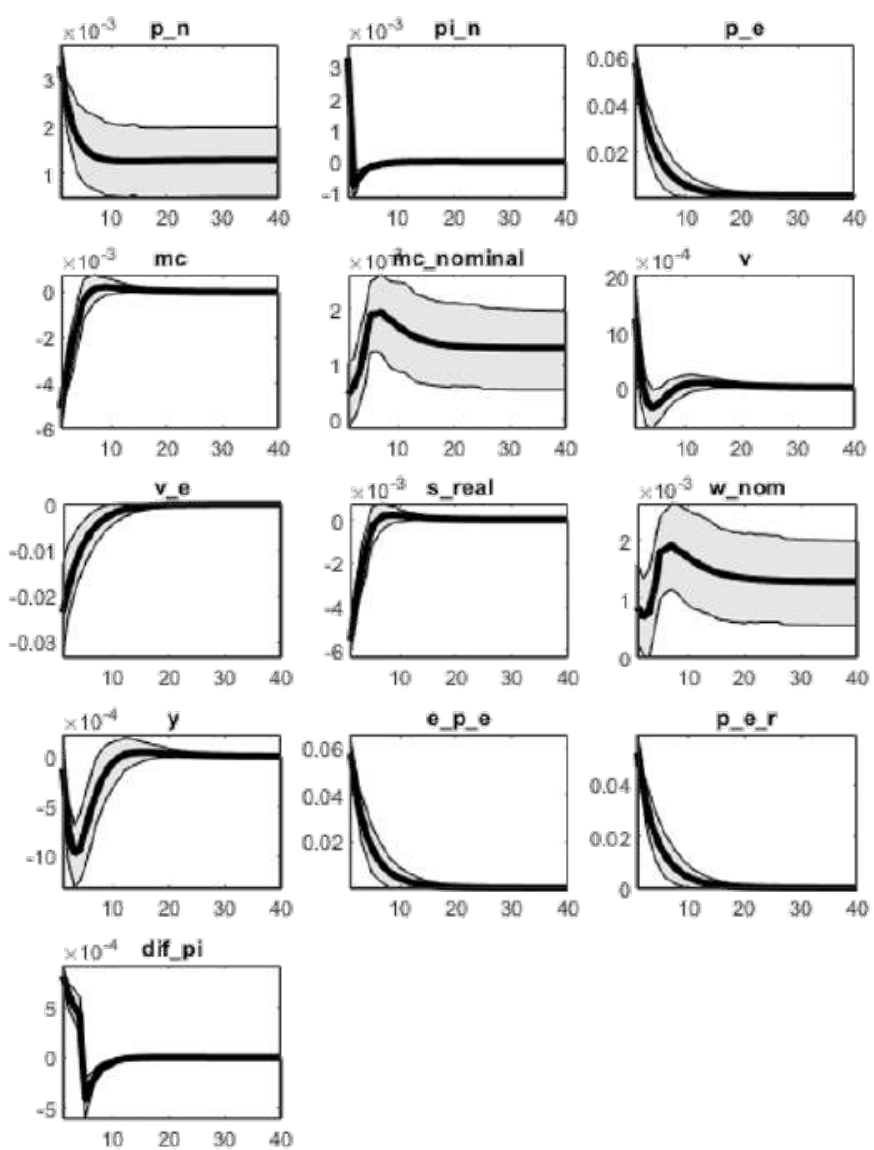
IRF: Choque de produtividade
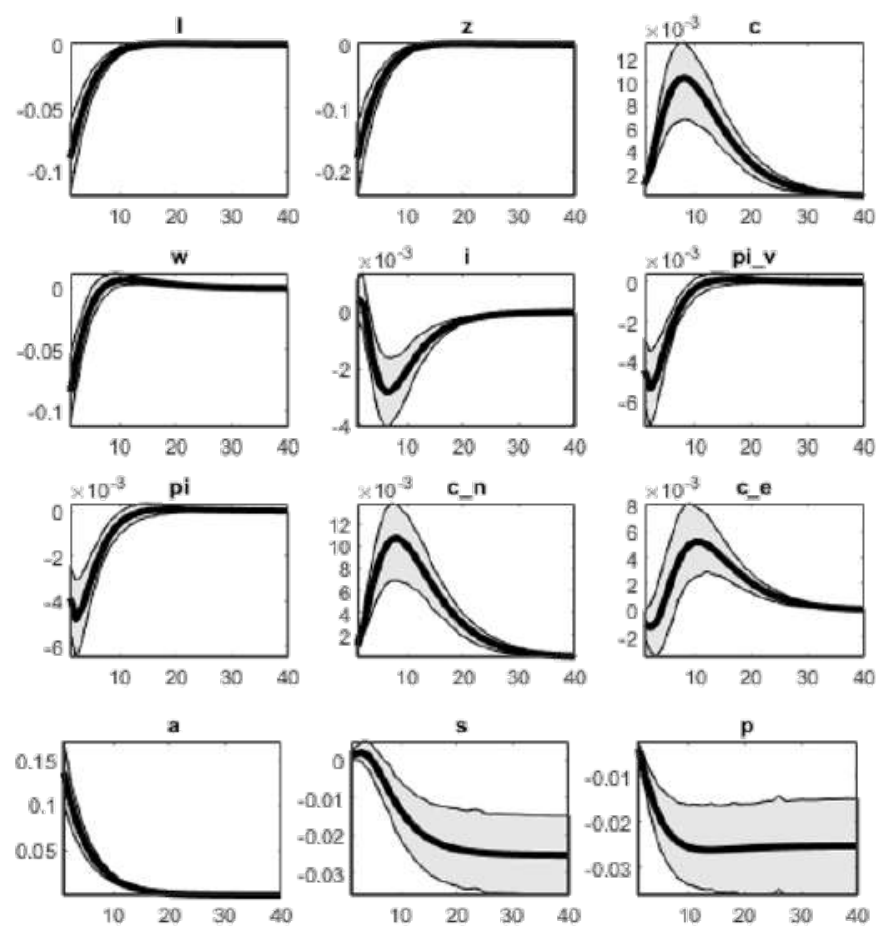

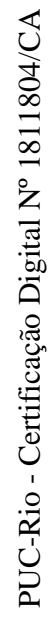
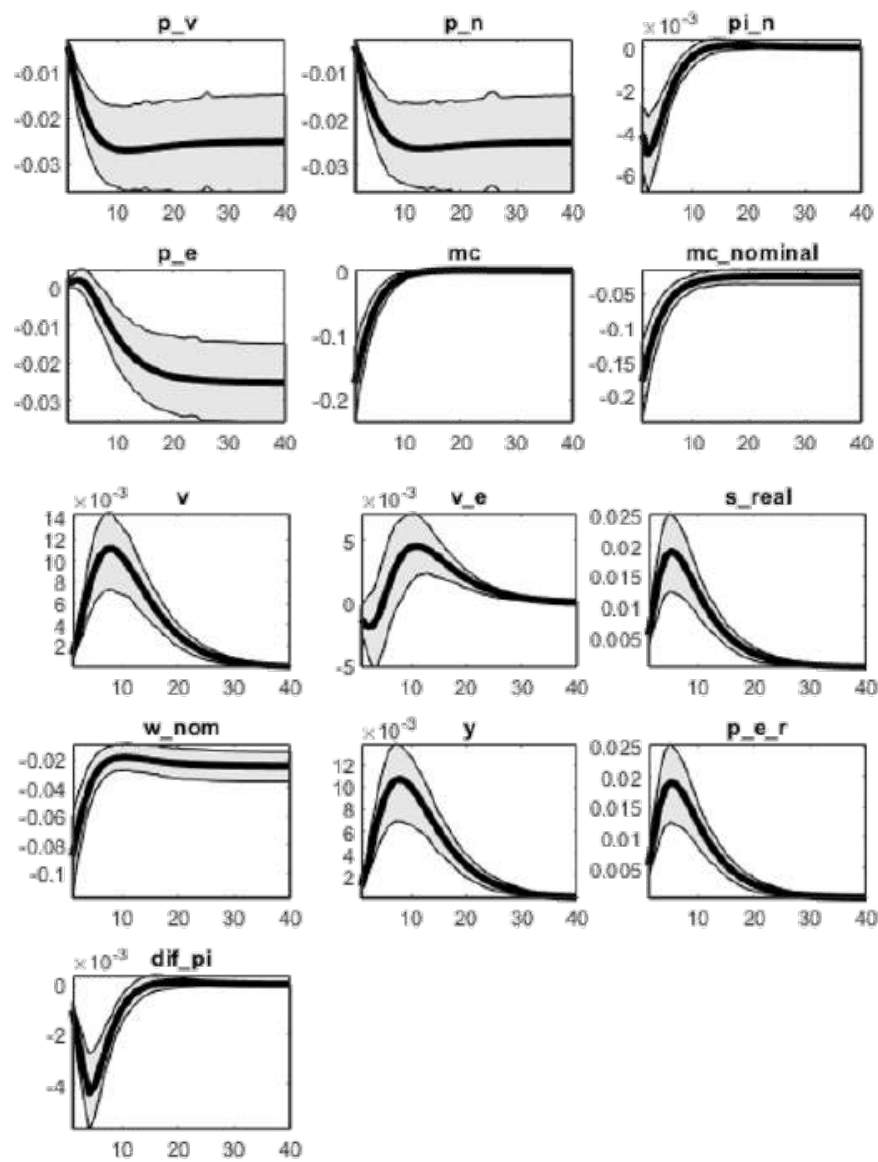
IRF: Choque de prêmio de risco
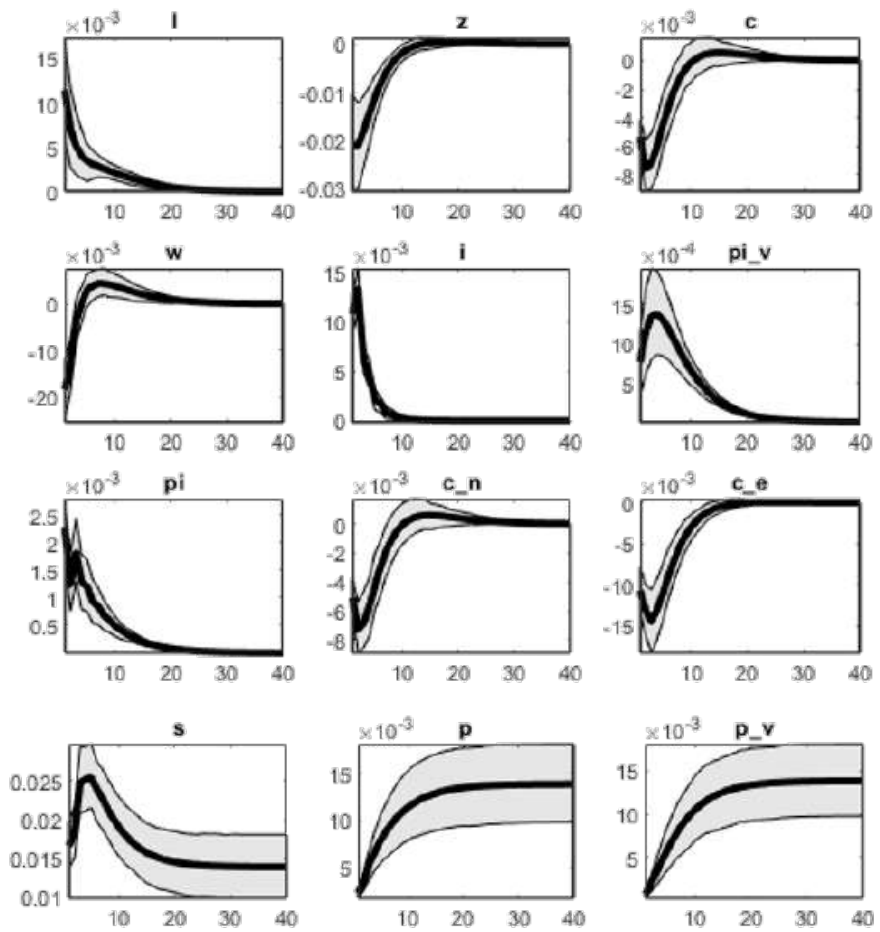

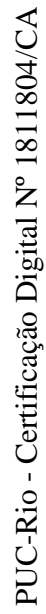
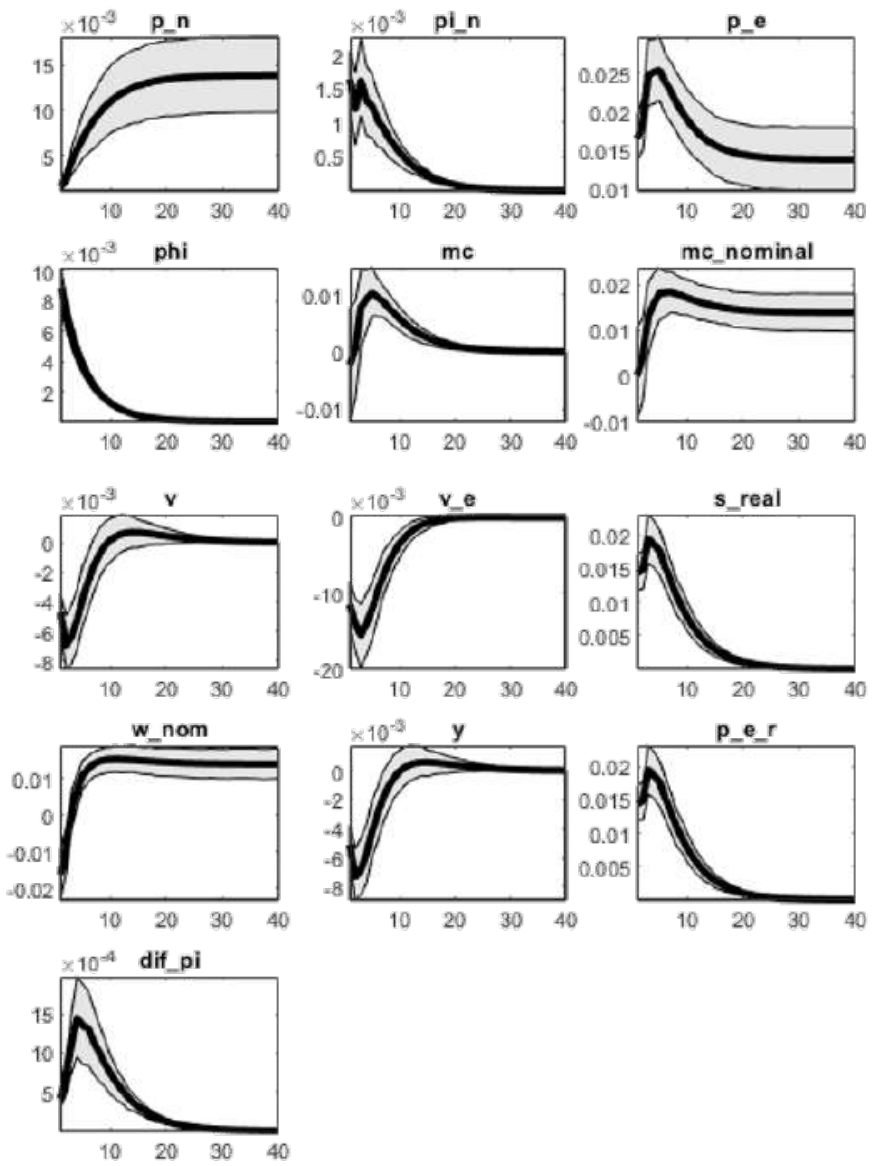
IRF: Choque na taxa de juros estrangeira
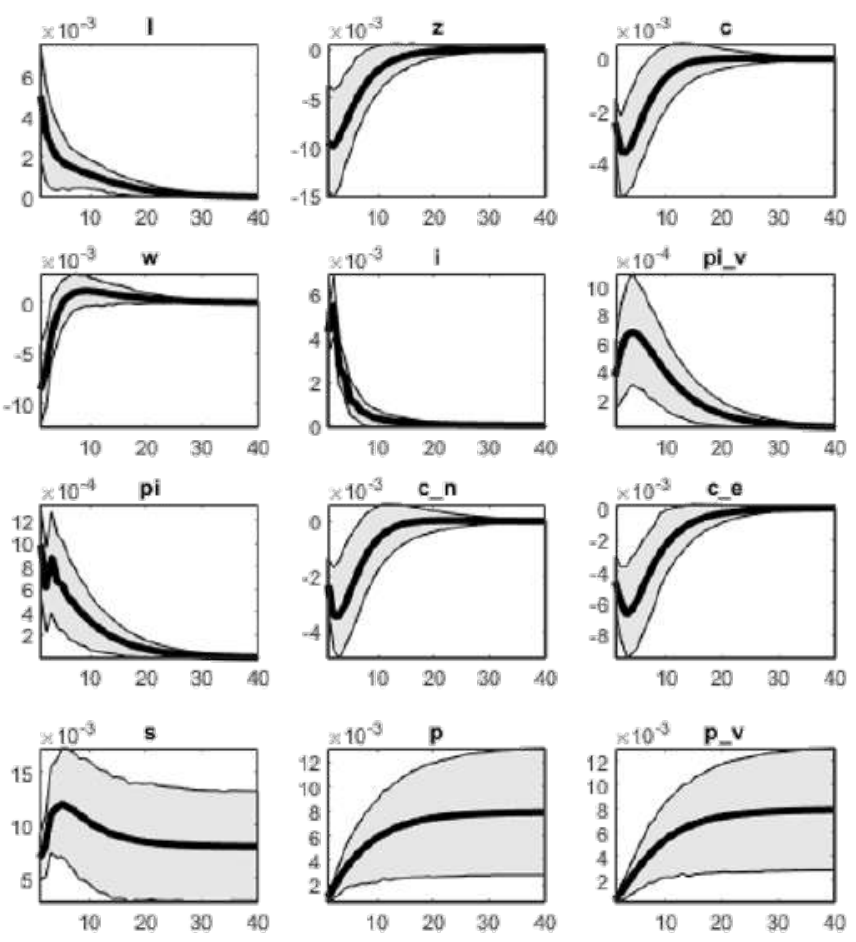

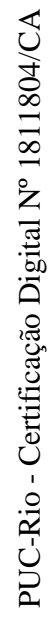
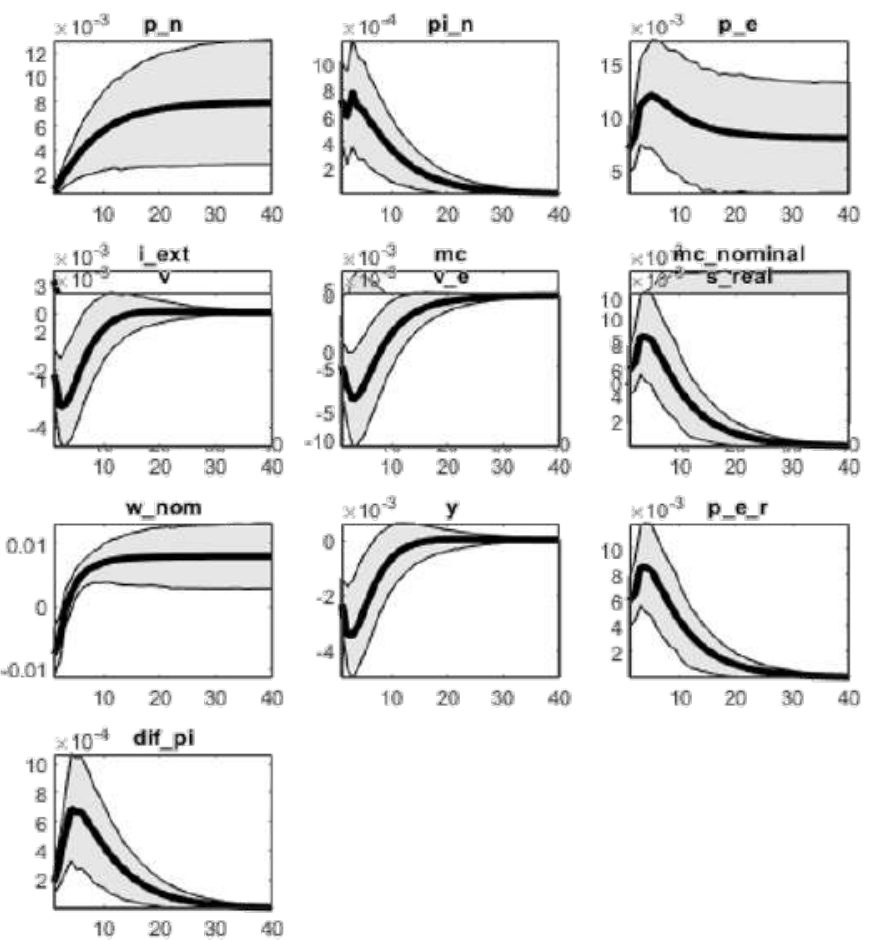


\section{6}

\section{Teste de robustez: série de dados começando em 2005}

O teste de robustez elaborado visa levar em consideração possíveis críticas ao fato da regra de politica monetária considerar a comparação da inflações passadas e futuras com a meta atual. Esta, por sua vez, seguiria um processo $\mathrm{AR}(1)$ e estaria sujeita a choques. Na realidade, o Conselho Monetário Nacional define em junho de cada ano a meta para a inflação de três anos-calendário à frente, sem possibilidade de choques nesse valor.

Diante de tal fato, optou-se por realizar o teste de robustez que consiste em estimar a alternativa de melhor aderência aos dados (Alternativa I: headline inflation e forward-looking) considerando apenas dados de 2005 a 2019, período onde a meta de inflação ficou praticamente constante no Brasil.

Como pode ser visto, há relativo aumento na sensibilidade da política monetária a desvios da inflação (de 2,16 para 2,46), e menor sensibilidade à variações no câmbio (de 1,05 a 0,93). Ainda, a persistência da taxa de juros é estimada ligeiramente maior (de 0,23 para 0,25 ).

Abaixo encontram-se os demais resultados.

\section{Forward-looking e headline inflation: MCMC diagnóstico multivariado}
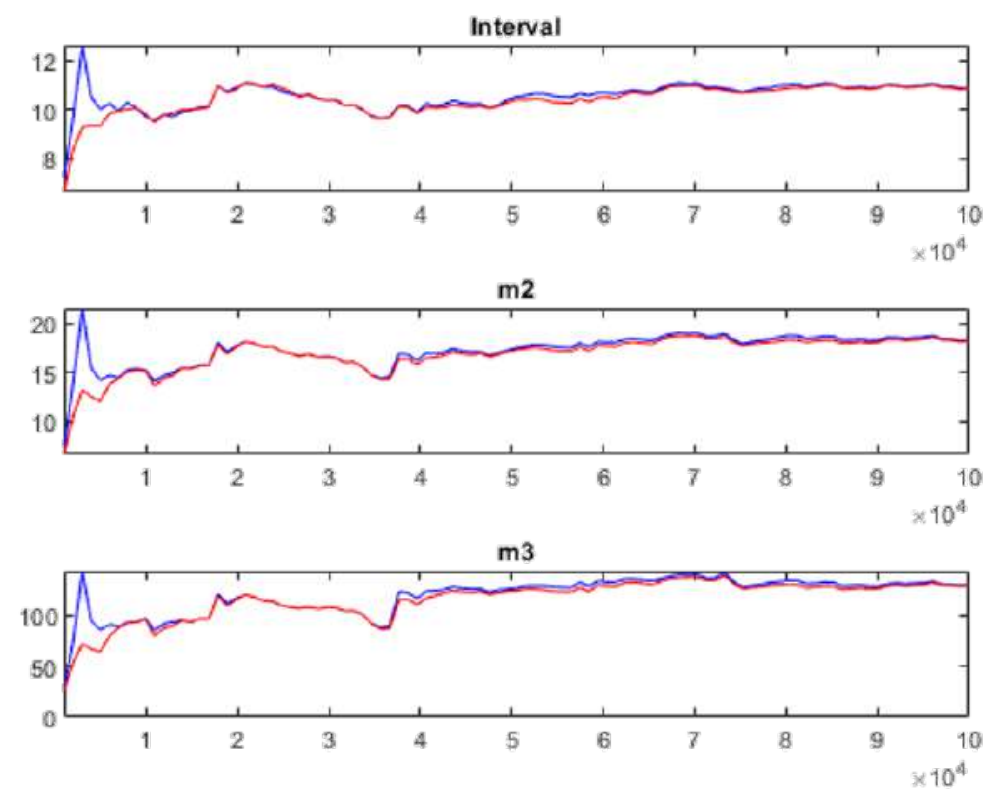
Historical x smoothed variables
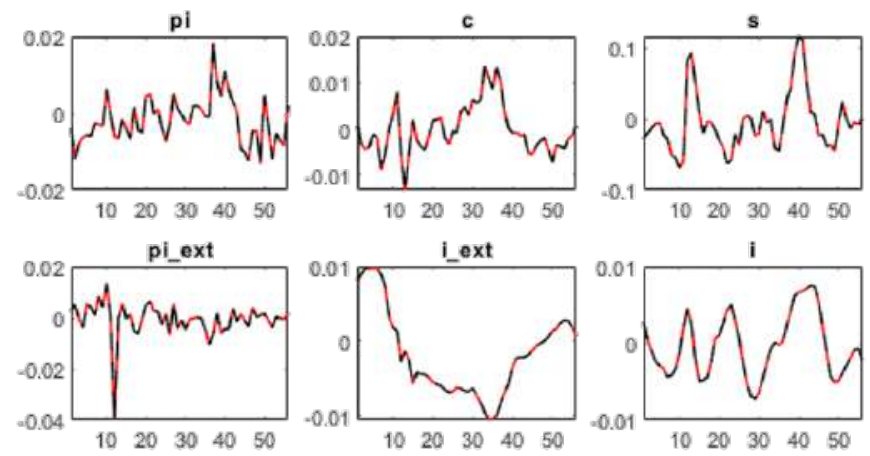

i_ext
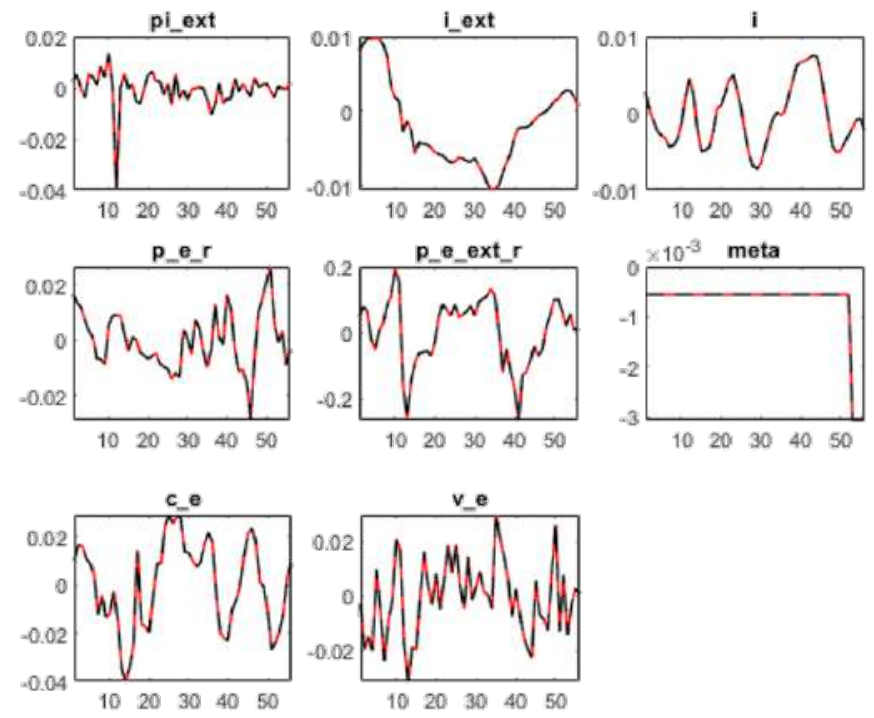

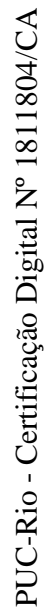

Smoothed shocks
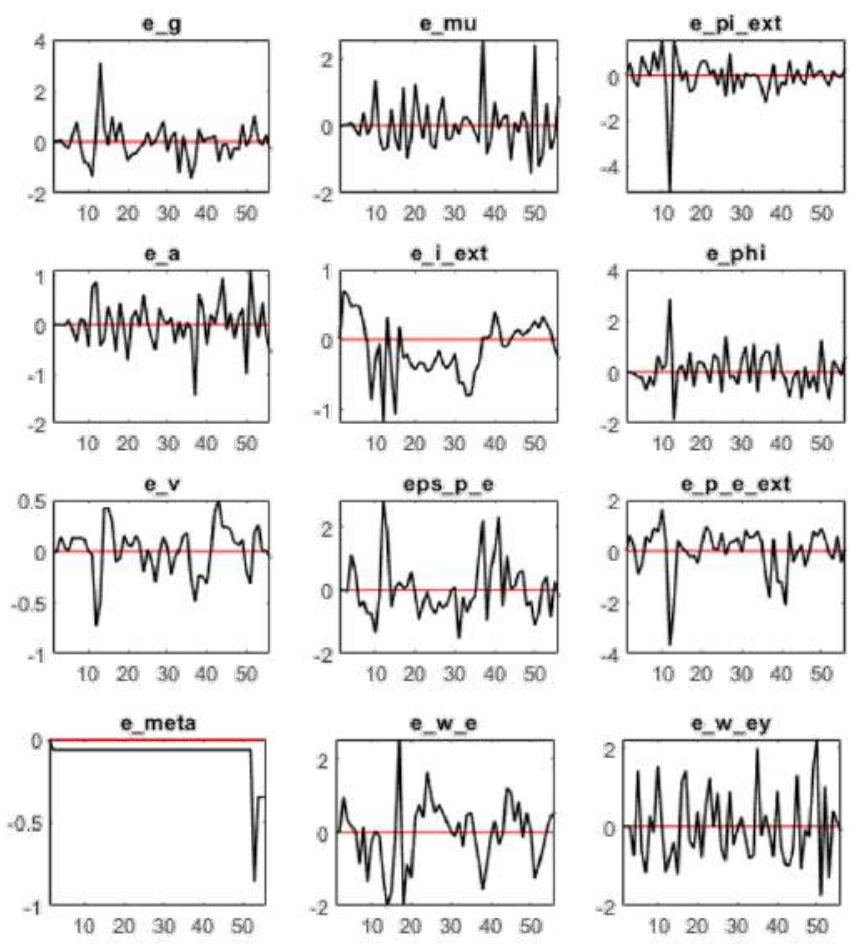
Teste de robustez - Forward-looking e headline inflation: Priors x posteriors
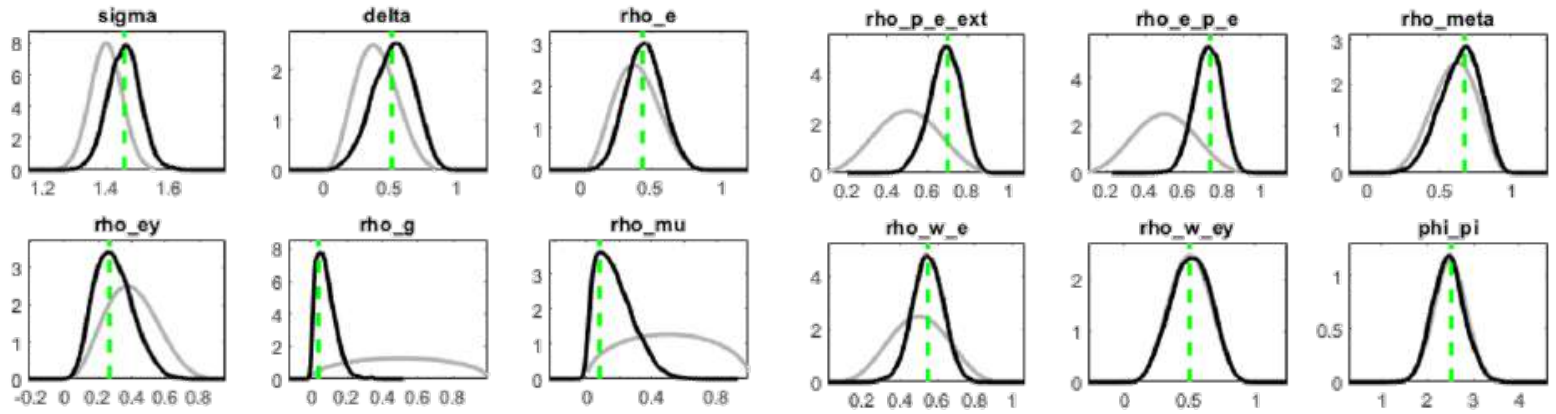

phi_pi
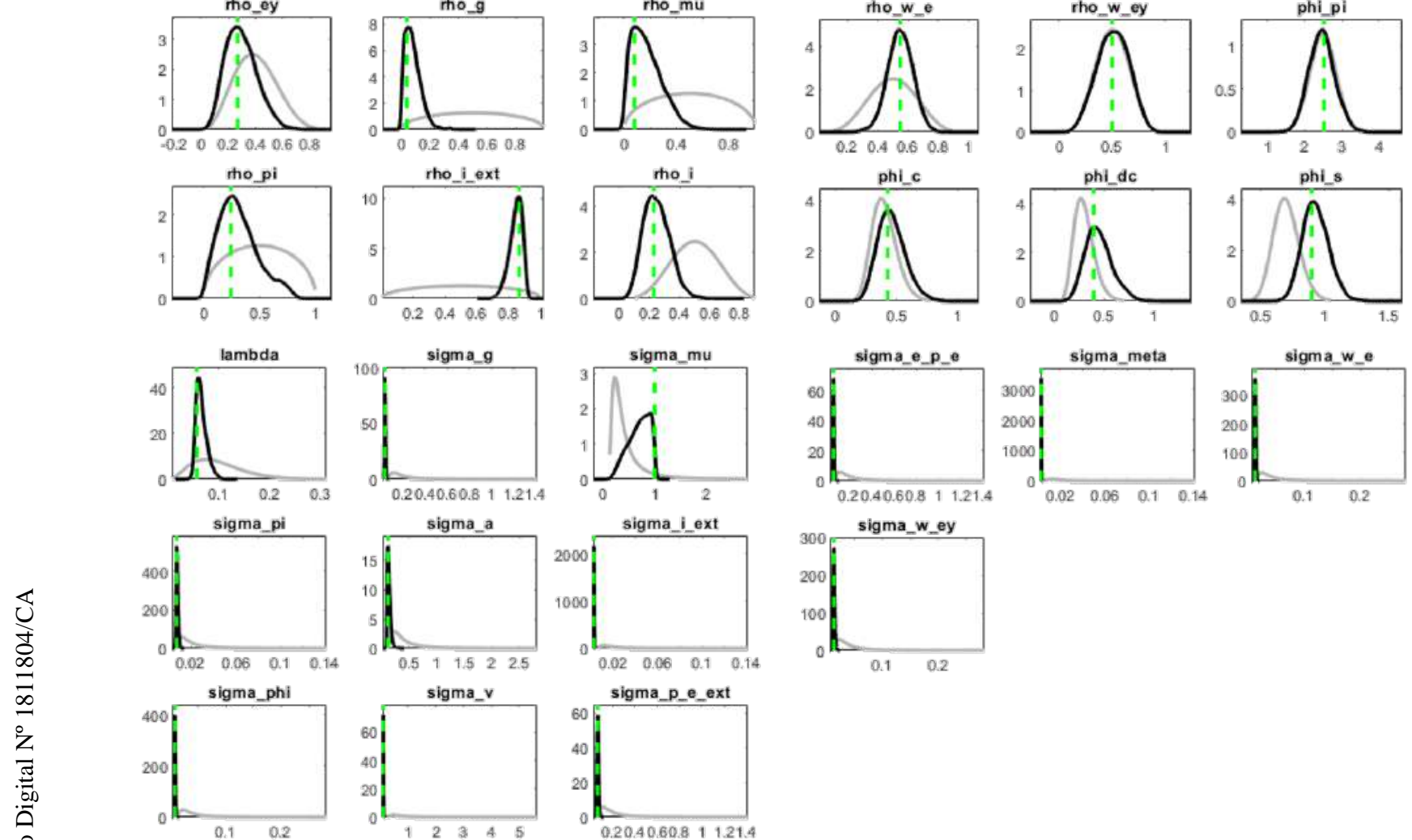

Z⿱ 
IRF: Choque de política monetária
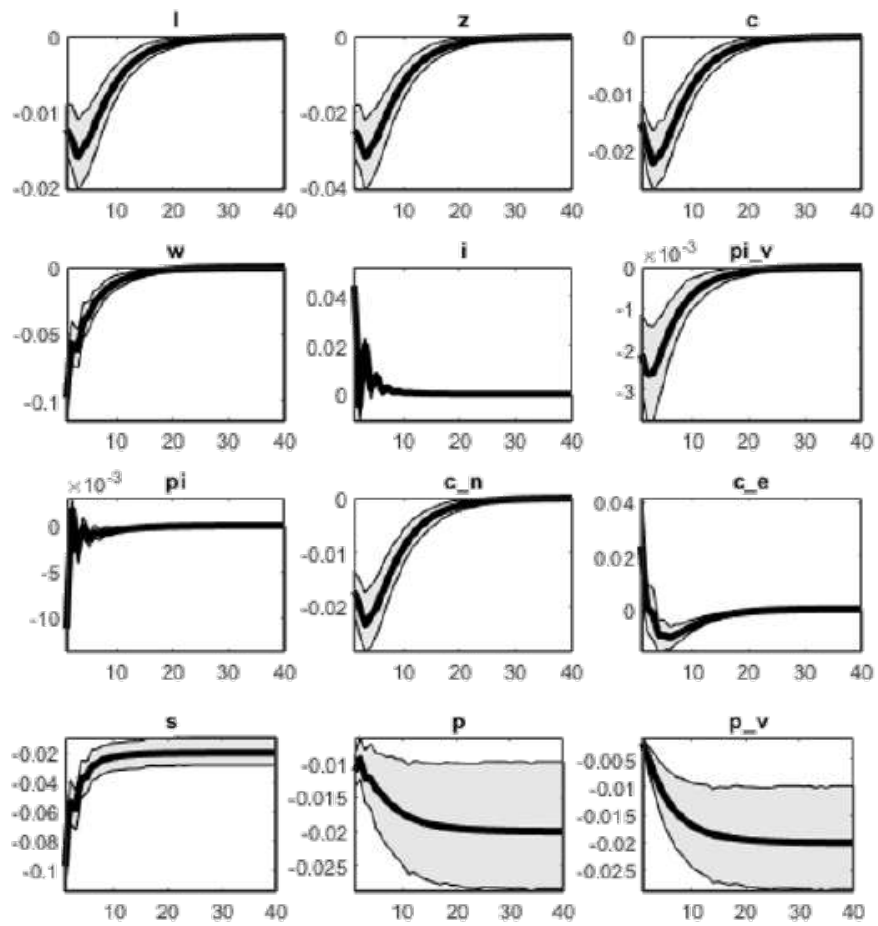

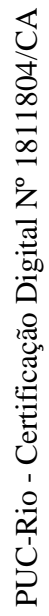
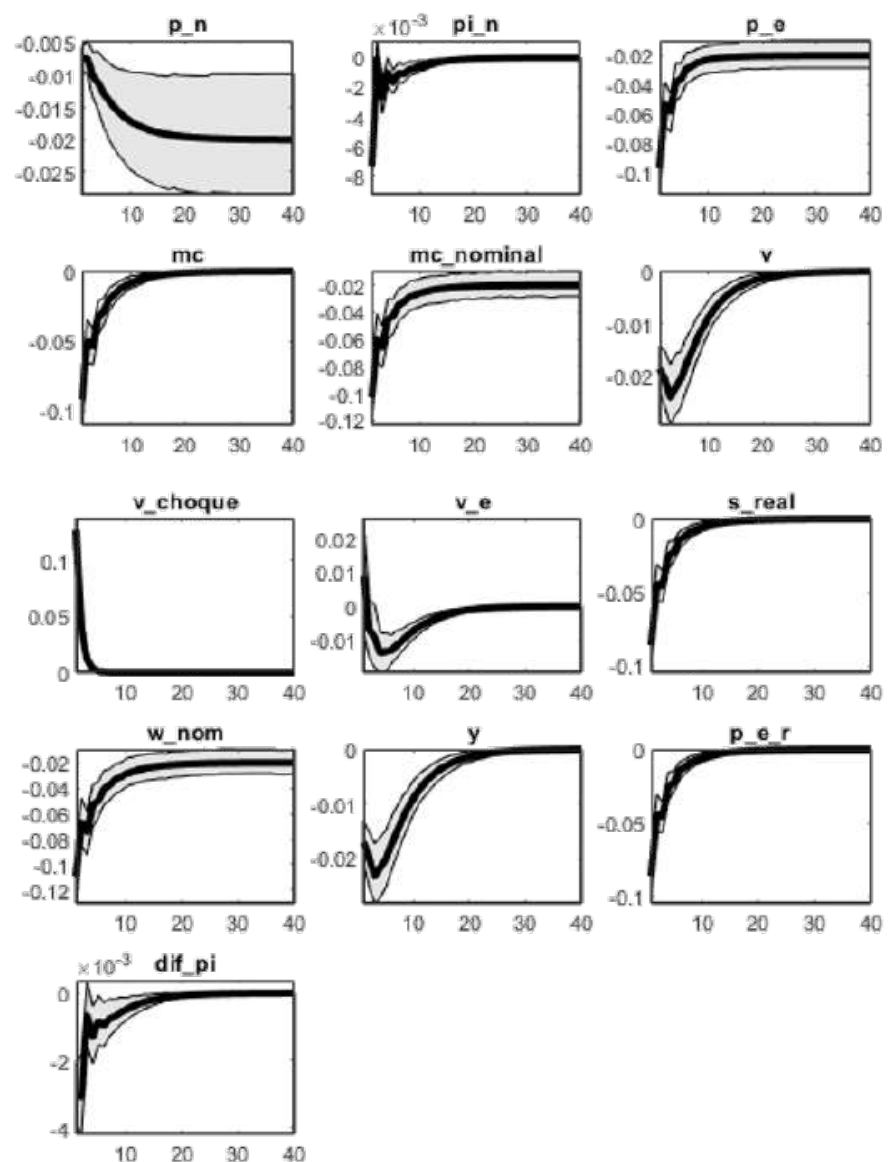
IRF: Choque nos preços internacionais de petróleo
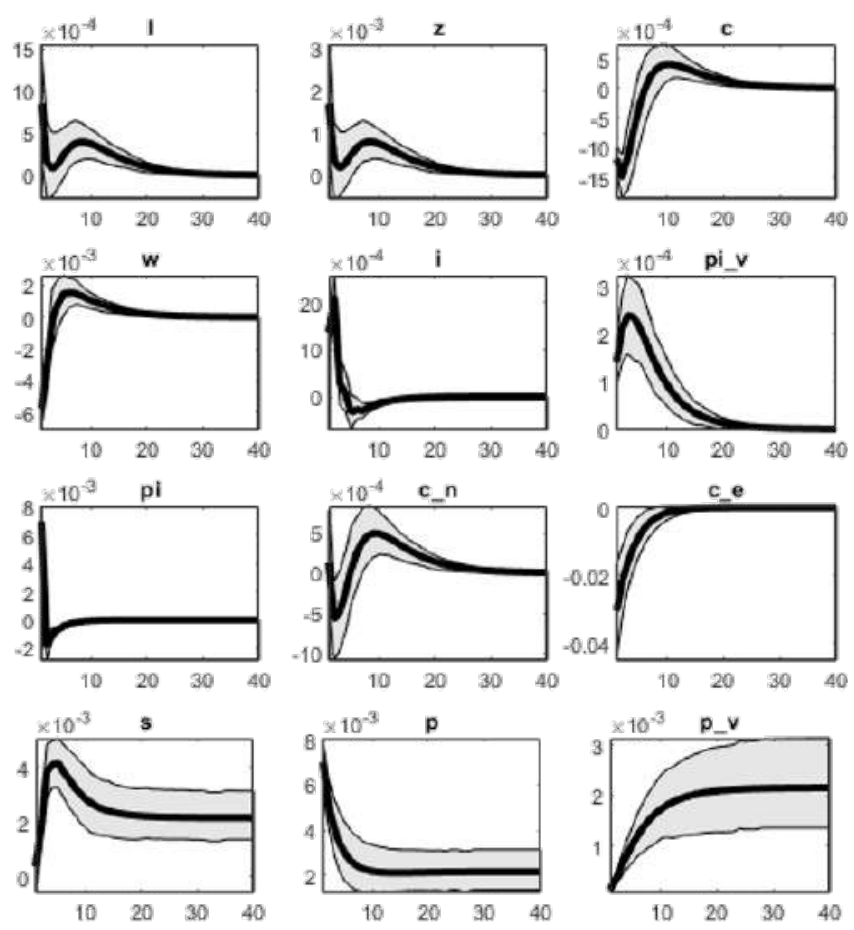

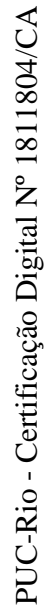
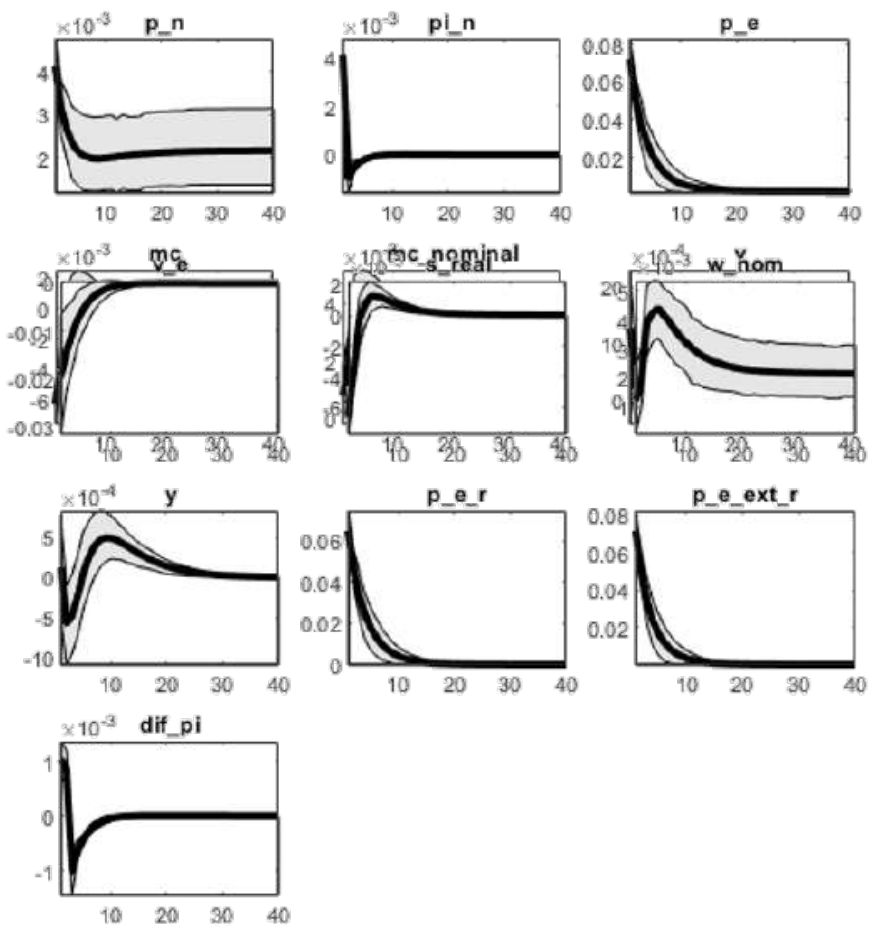
IRF: Choque nos preços derivados nacionais
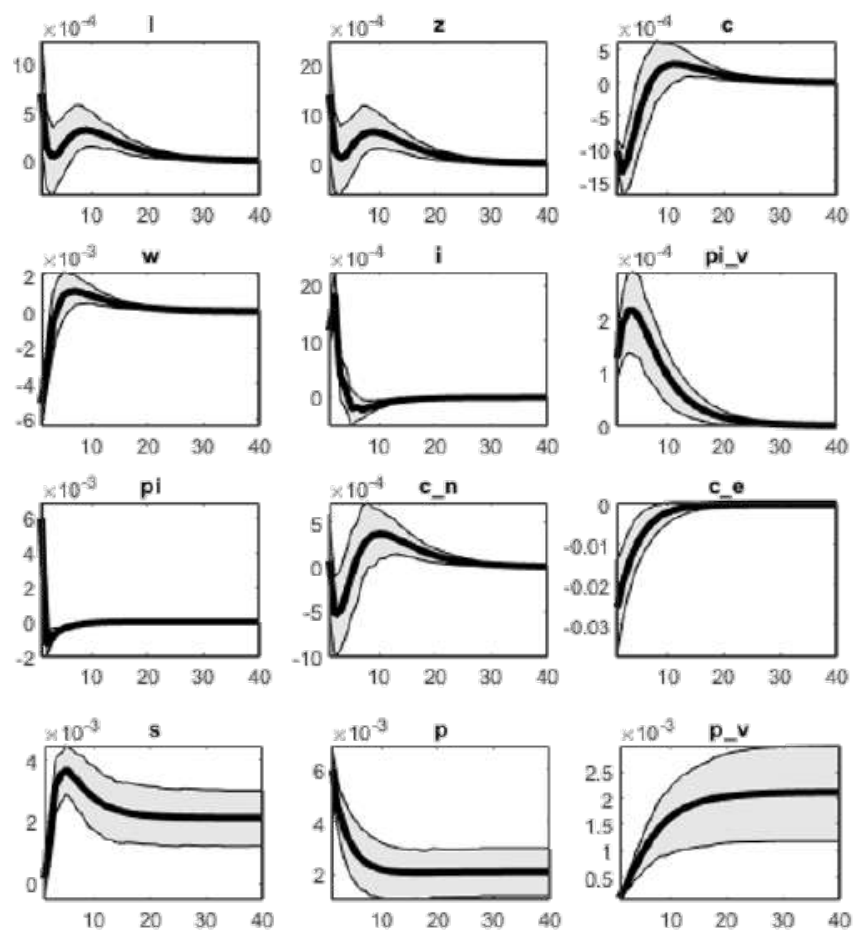

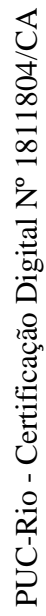
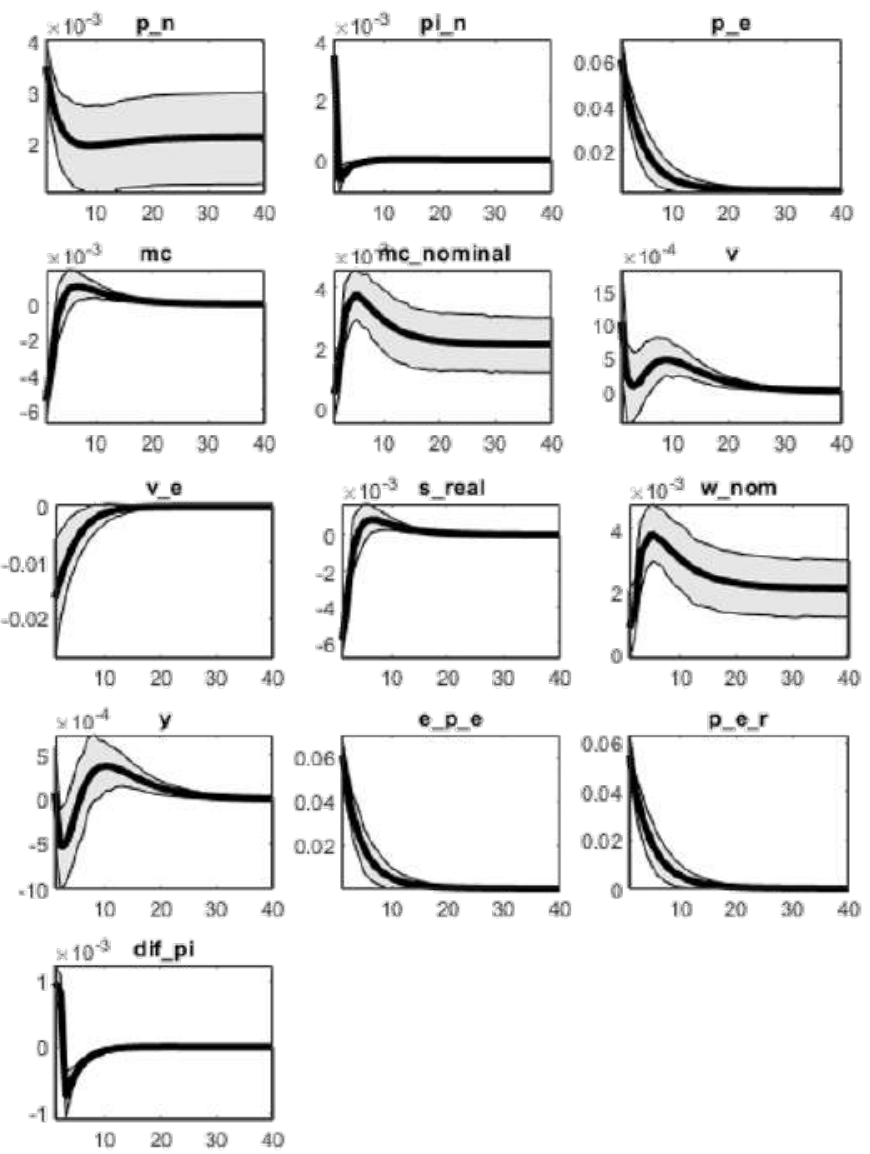
IRF: Choque de produtividade
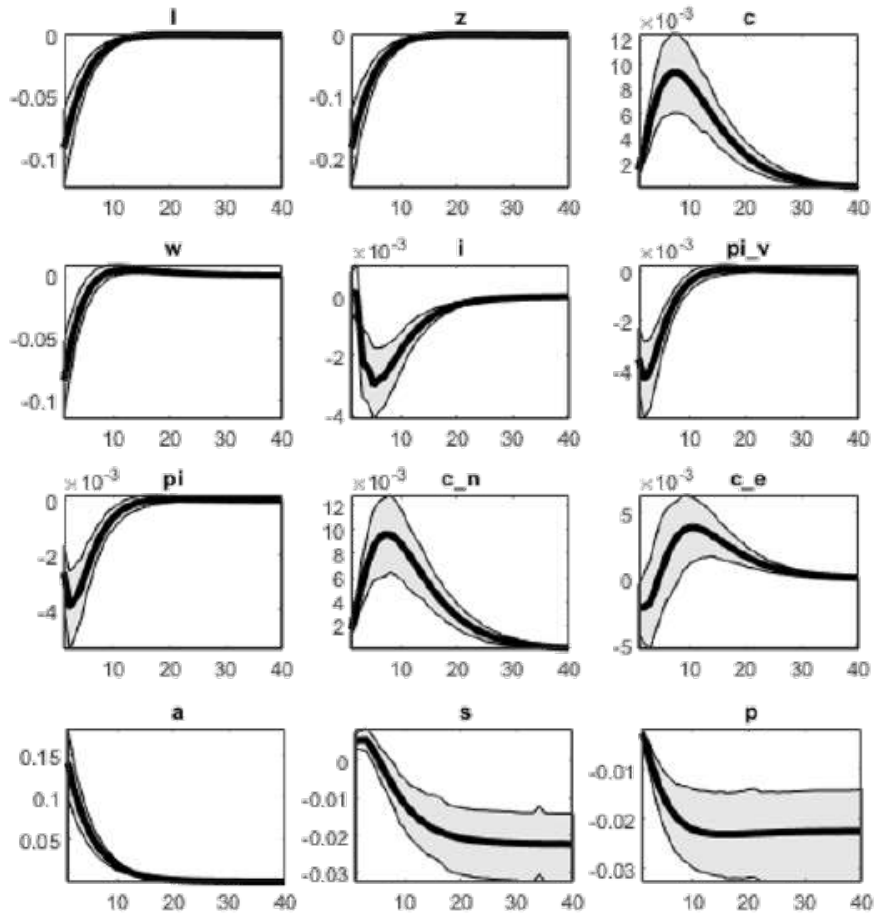

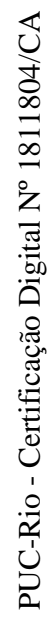
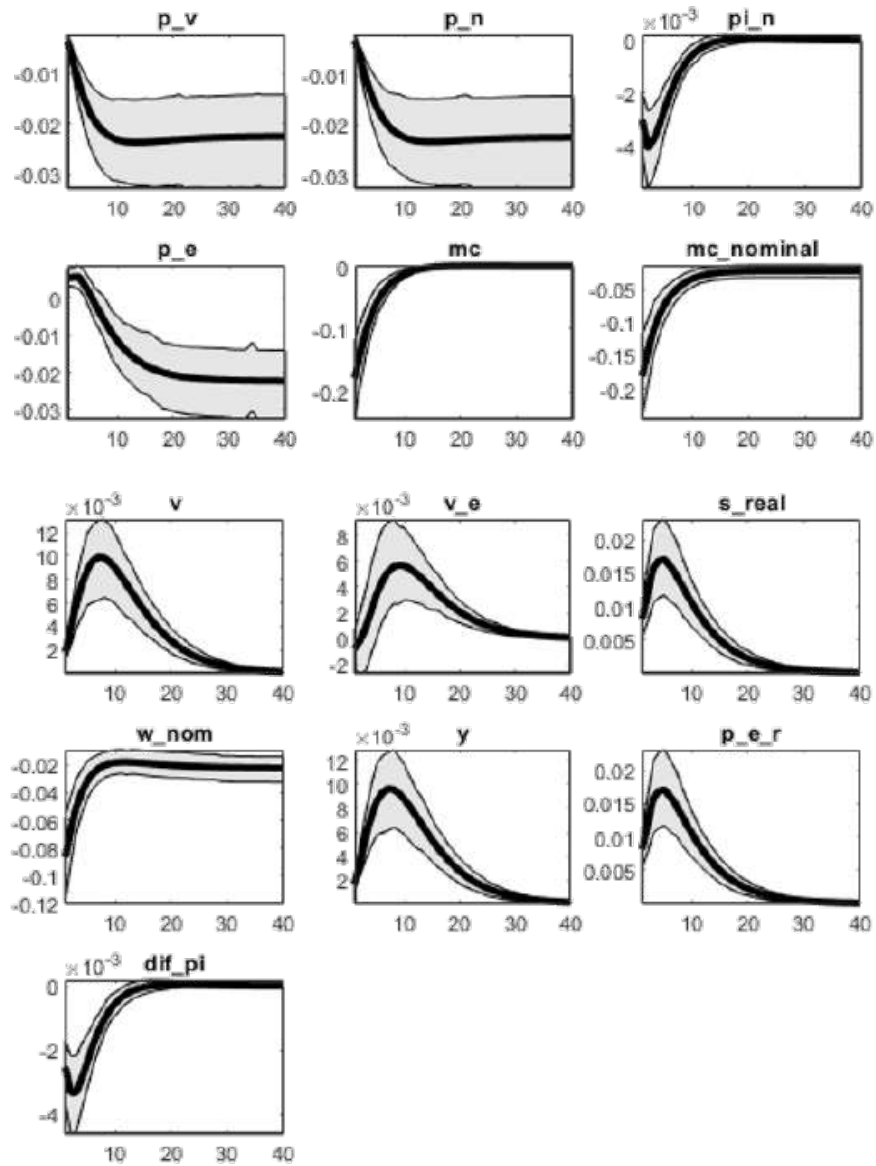
IRF: Choque de prêmio de risco
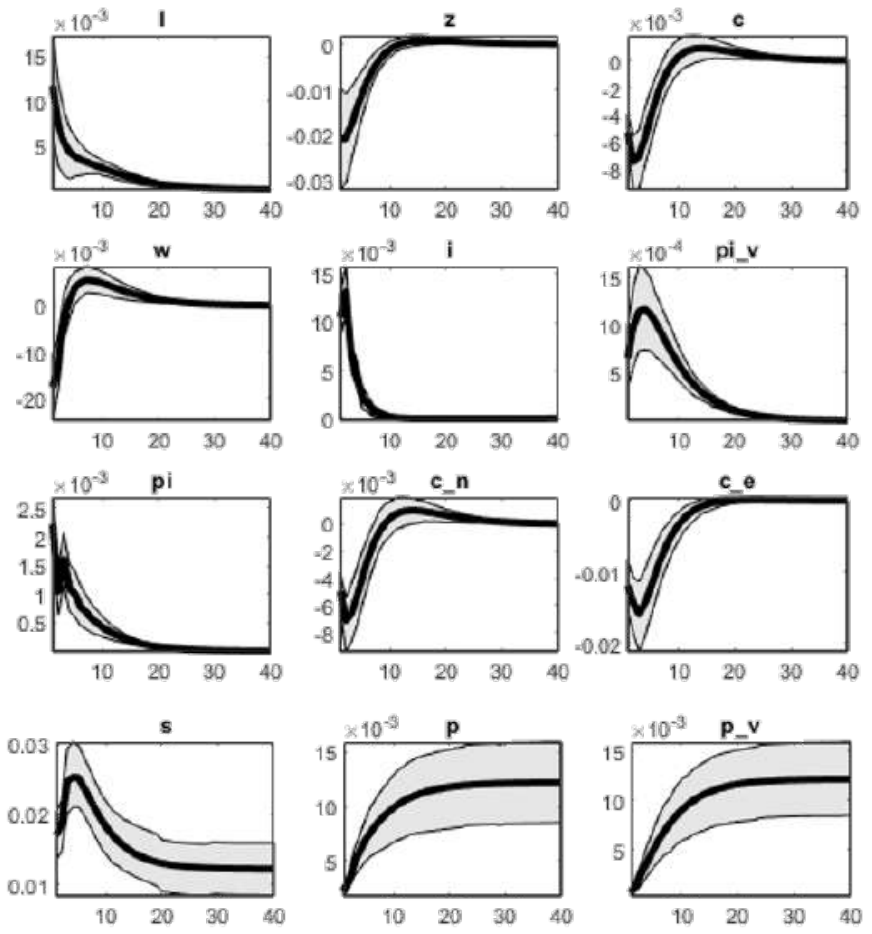

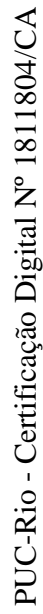
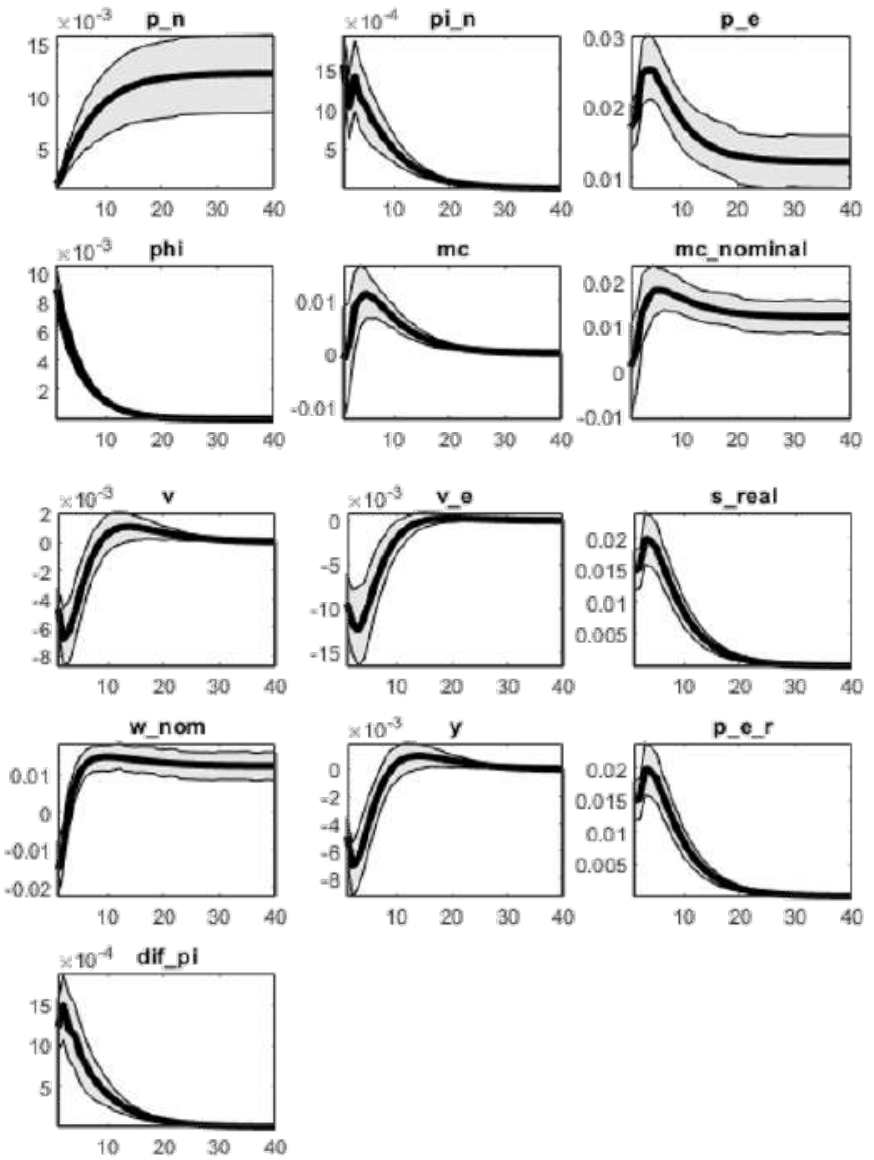
IRF: Choque na taxa de juros estrangeira
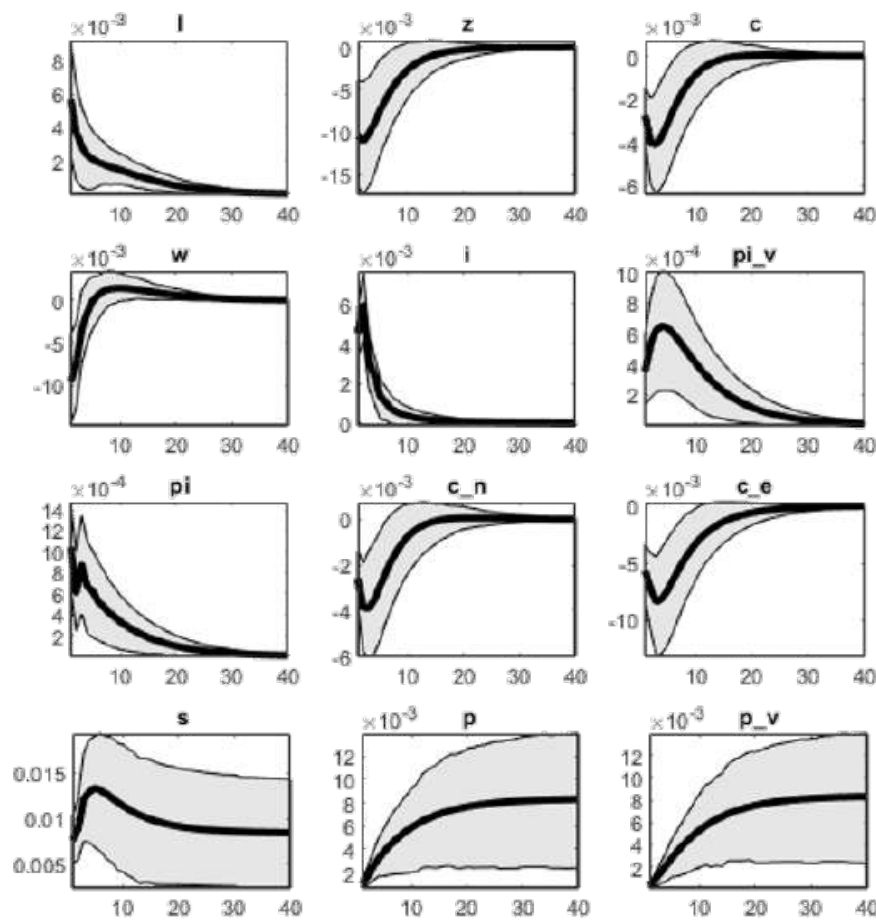

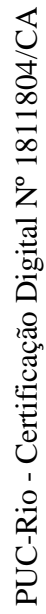
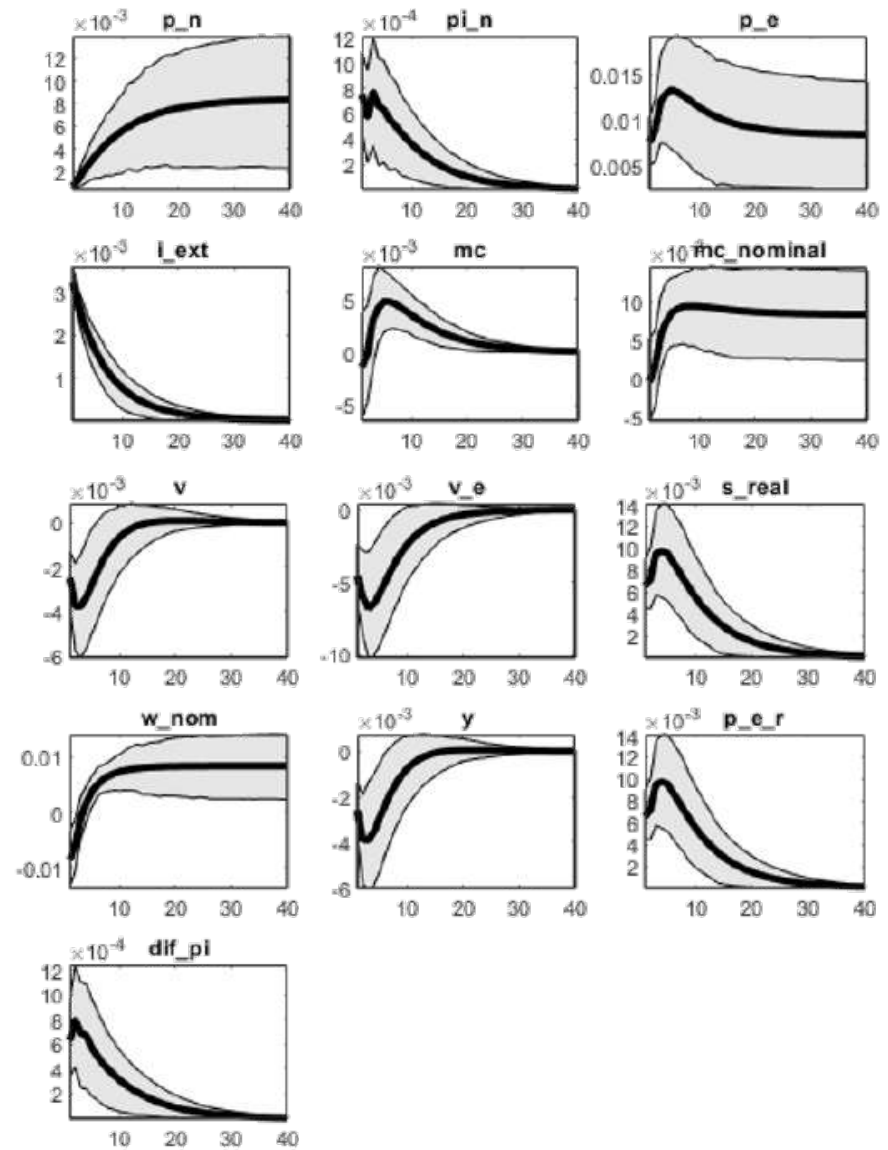


\section{7}

\section{Versão com calibração de parâmetro não identificado}

Por meio da observação dos gráficos que comparam priors x posteriors, foi possível observar que o parâmetro $\rho_{w_{e y}}$ não estaria identificado, por apresentar distribuição prior e posterior praticamente idêntica.

Ao analisar as possíveis causas de tal fato, acreditamos que tenha ocorrido devido à existência de apenas uma variável observável entre as presentes na cesta de insumos das firmas produtoras de bens finais, sendo ela a demanda por energéticos das firmas $\left(V_{e}\right)$. Já o parâmetro $\rho_{w_{e}}$ foi corretamente identificado, tendo como base as duas variáveis observáveis contidas na cesta de bens das famílias: $C_{e}$ e $C$.

Entre as potenciais soluções para tal fato estão a calibração do parâmetro $\rho_{w_{e y}}$ com base no valor estimado para $\rho_{w_{e}}$, o que foi feito sem alterações nos resultados. Estes podem ser encontrados abaixo, referindo-se à regra de política monetária que mira inflação headline e forward-looking:

\section{MCMC diagnóstico multivariado}

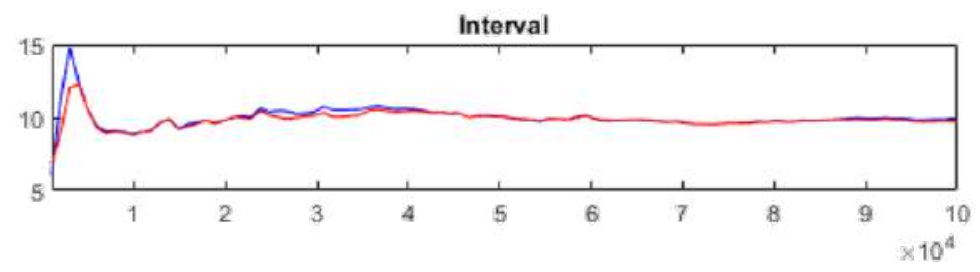

m2

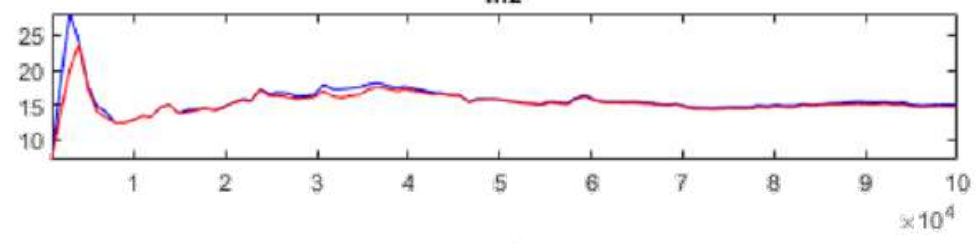

m3

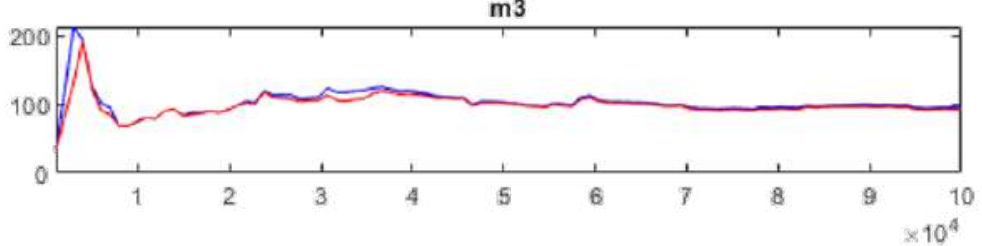


Historical x smoothed variables
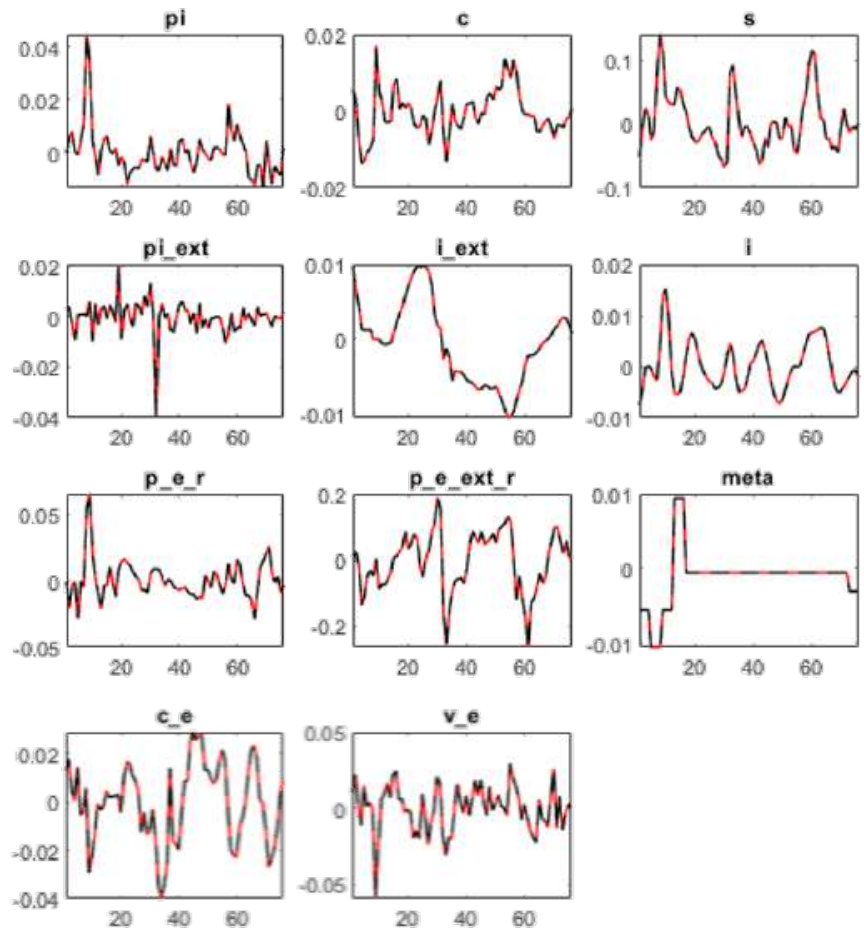

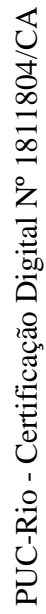

Smoothed shocks
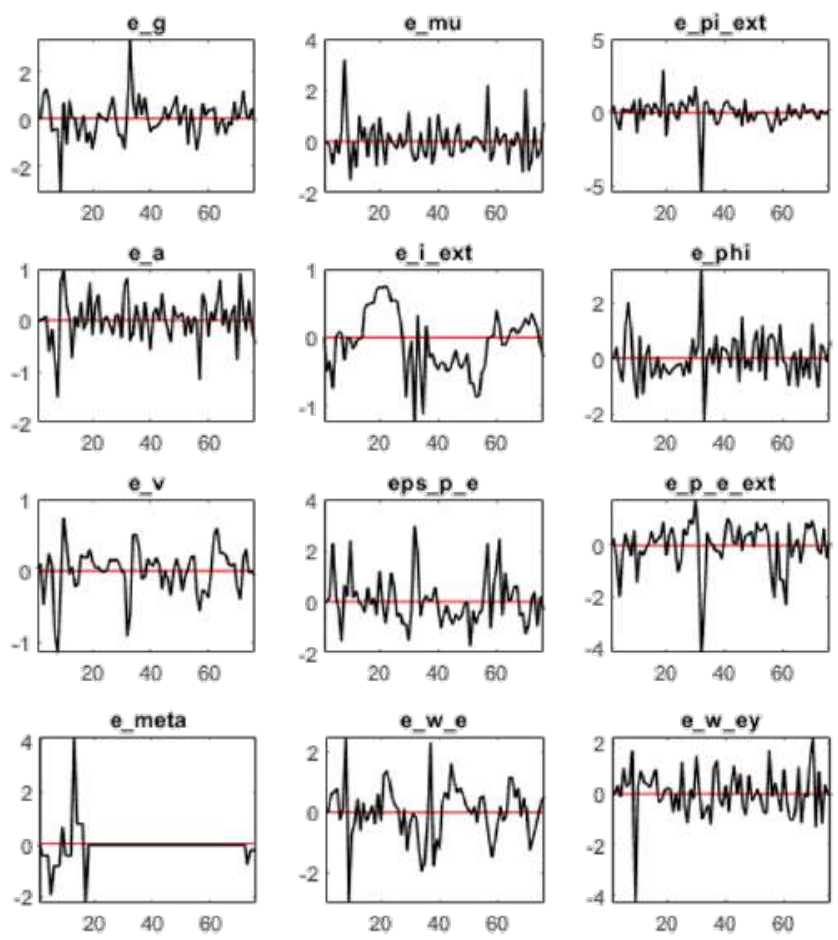
Priors $\mathrm{x}$ posteriors
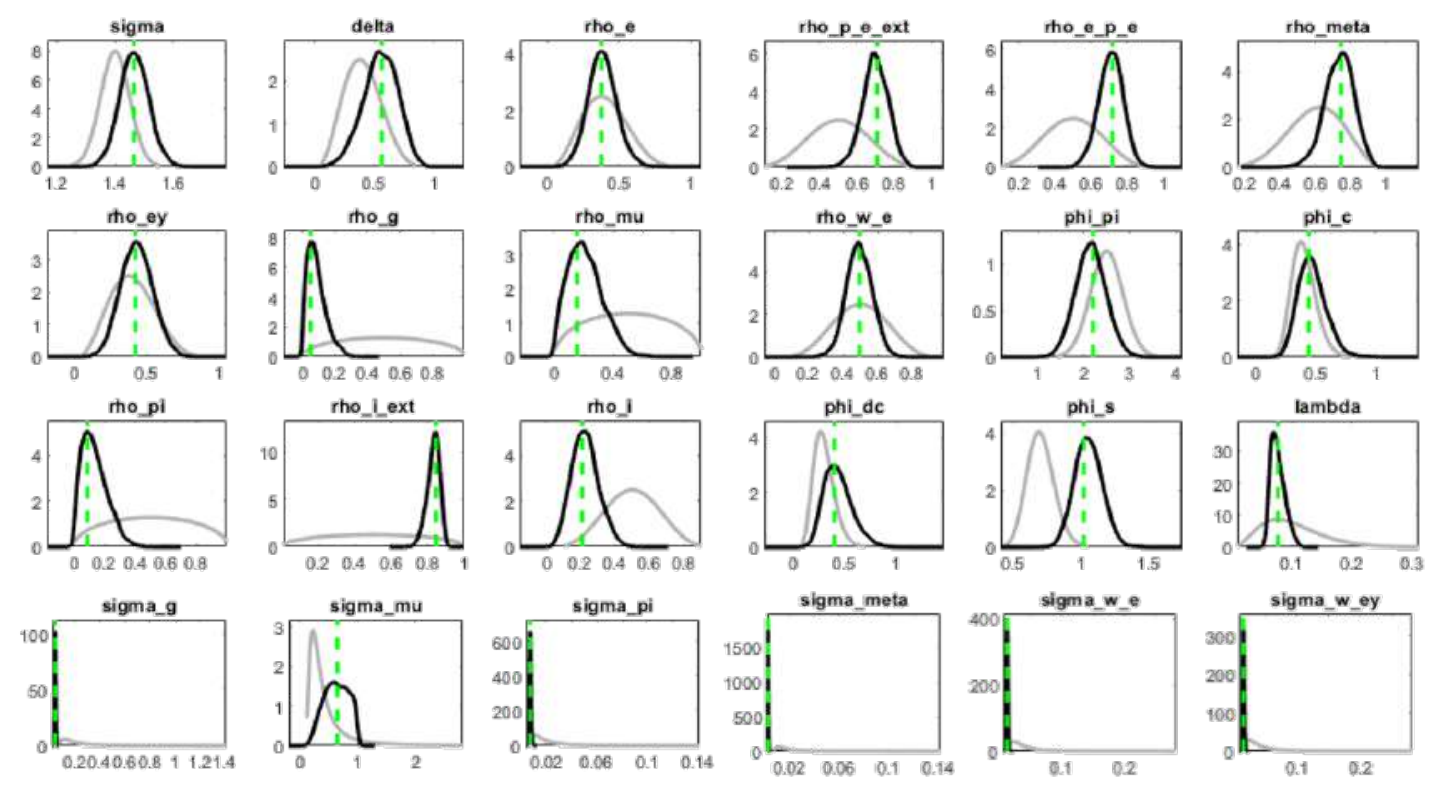

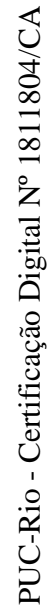
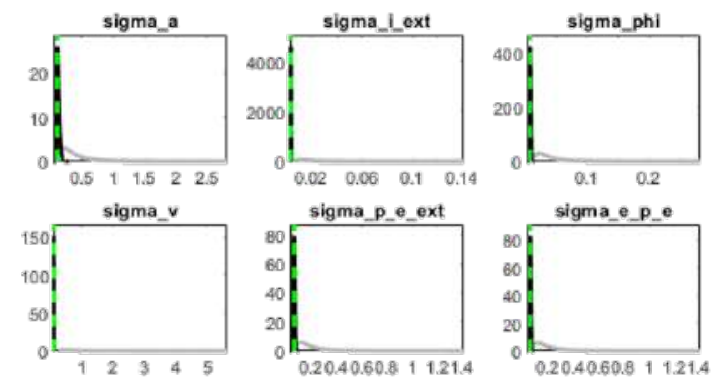
IRF: Choque de política monetária
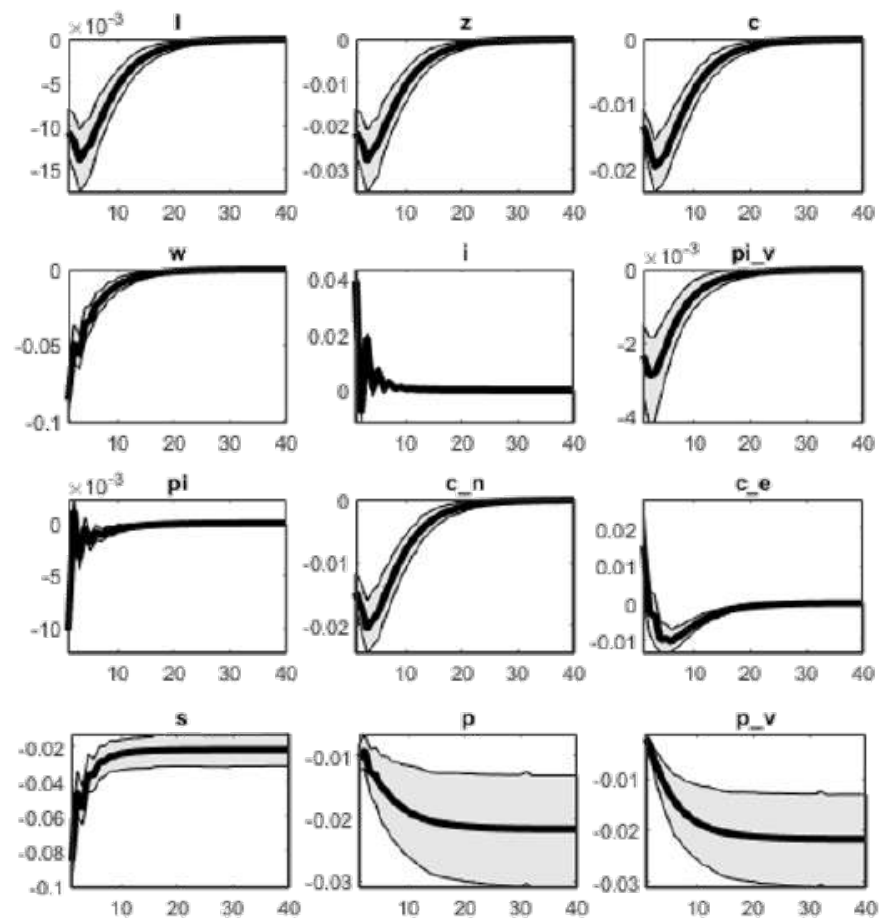

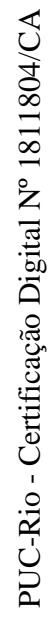
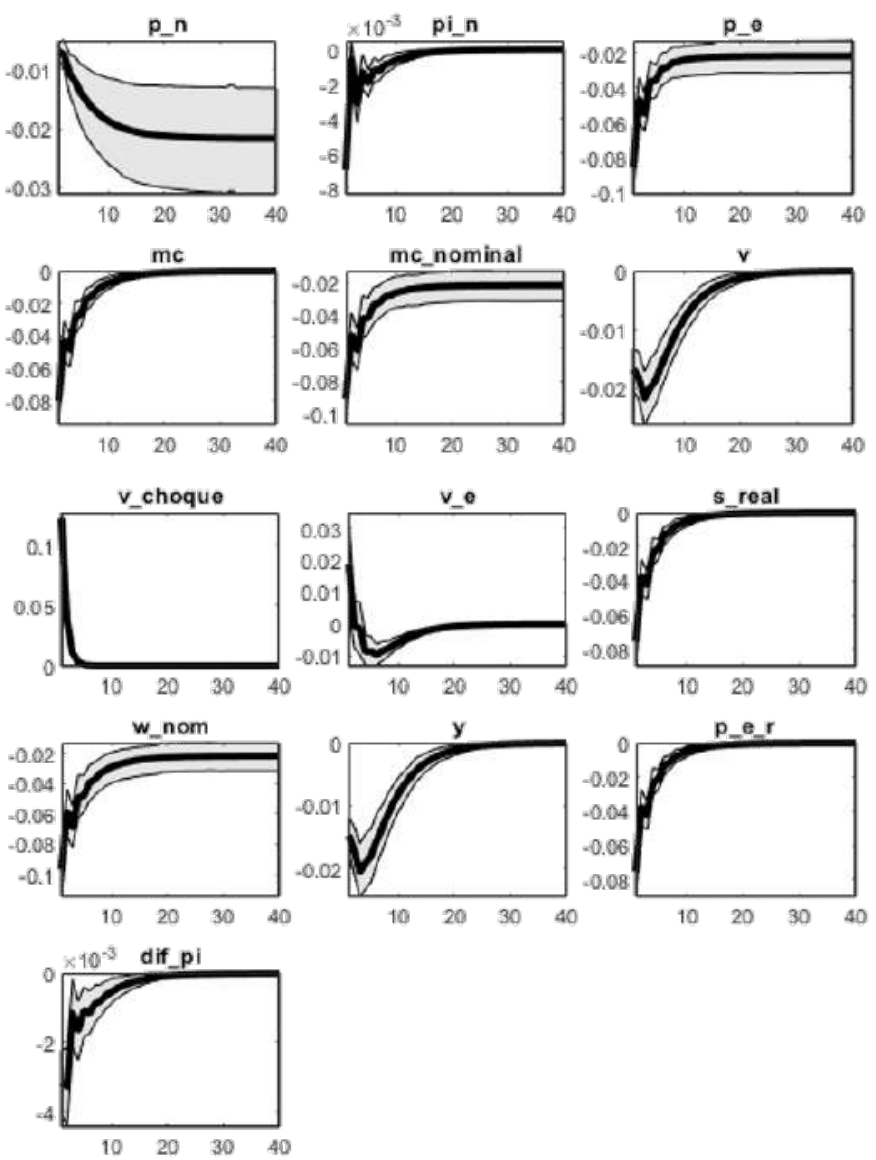
IRF: Choque nos preços internacionais de petróleo
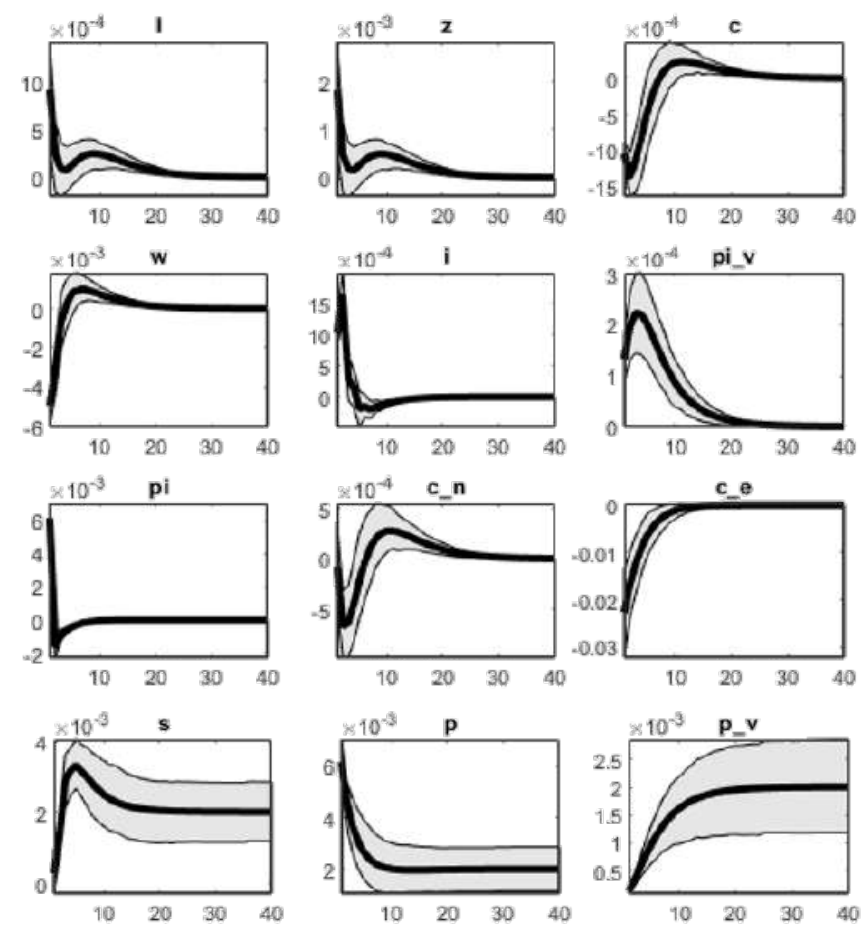

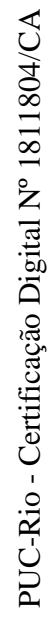
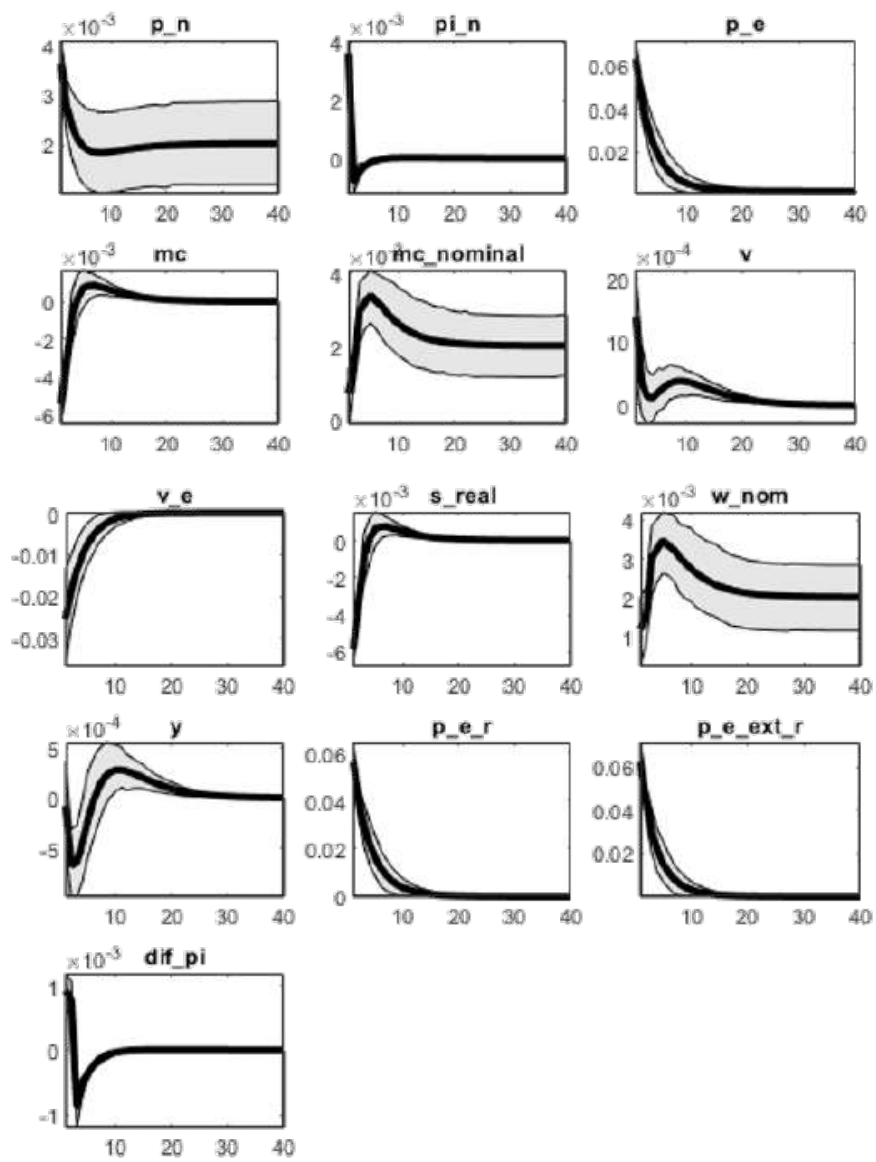
IRF: Choque nos preços de derivados nacionais
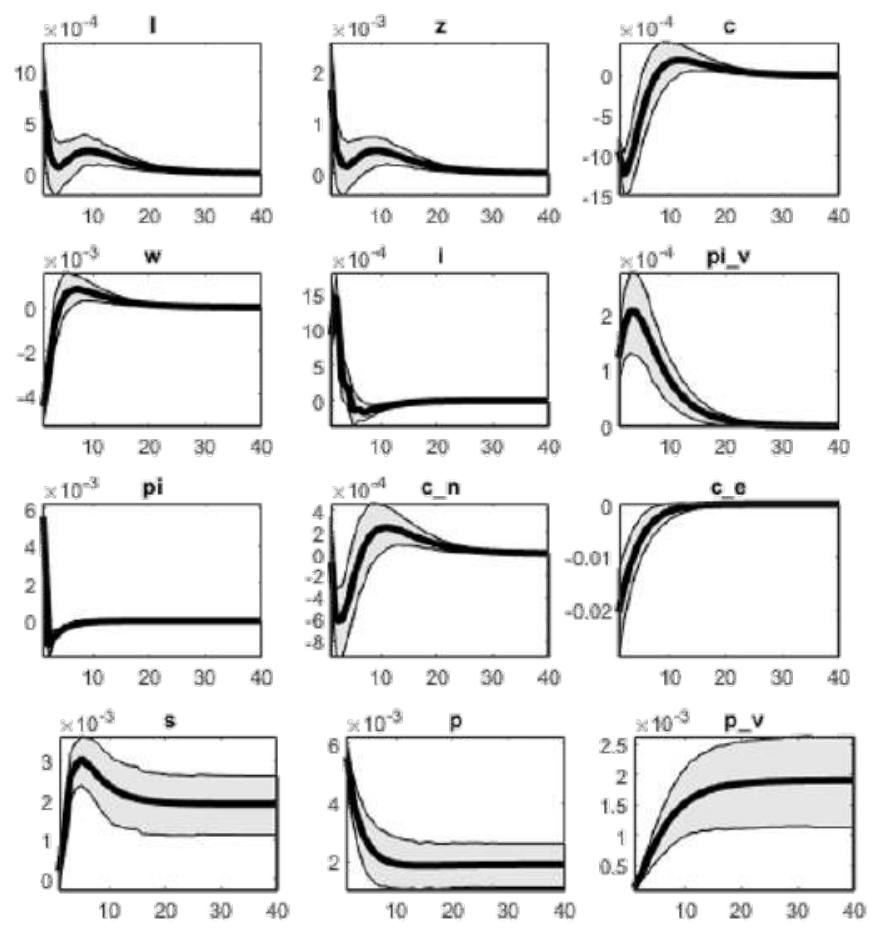

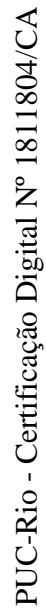
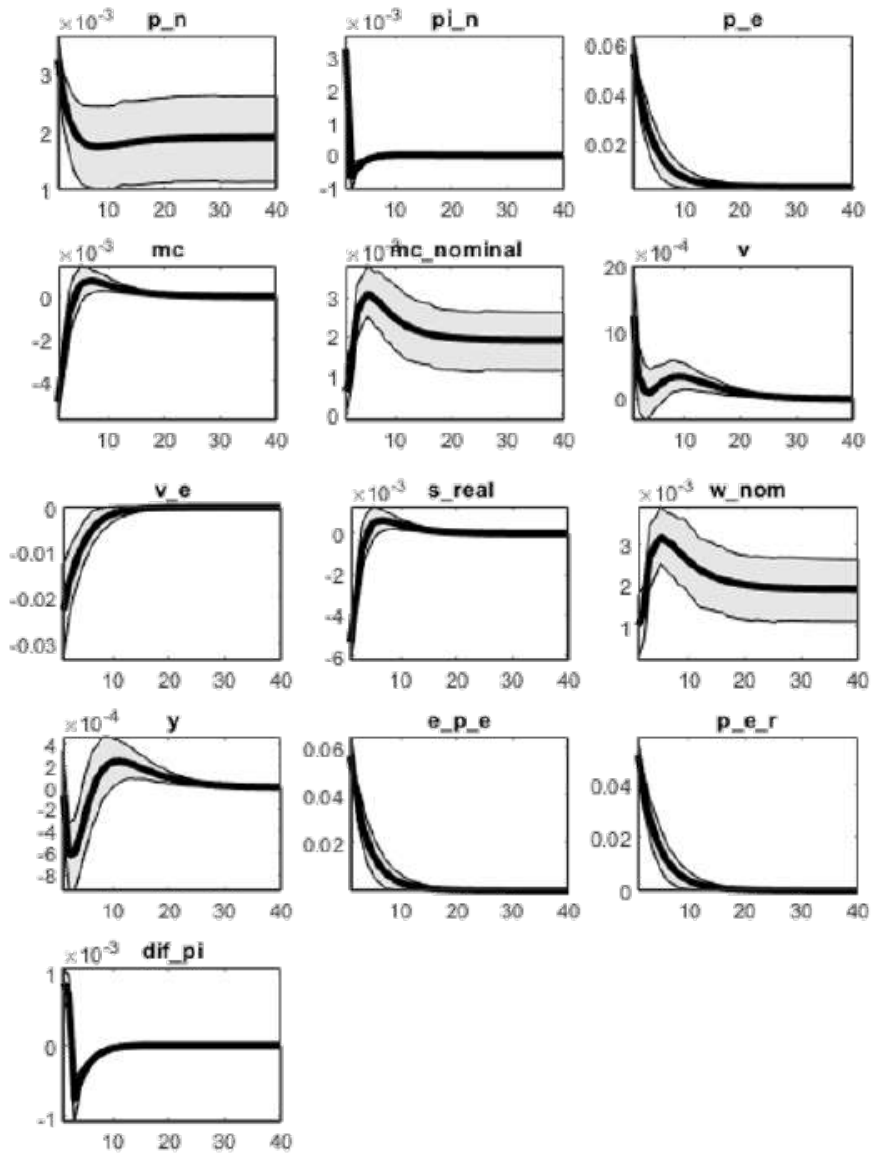
IRF: Choque de produtividade
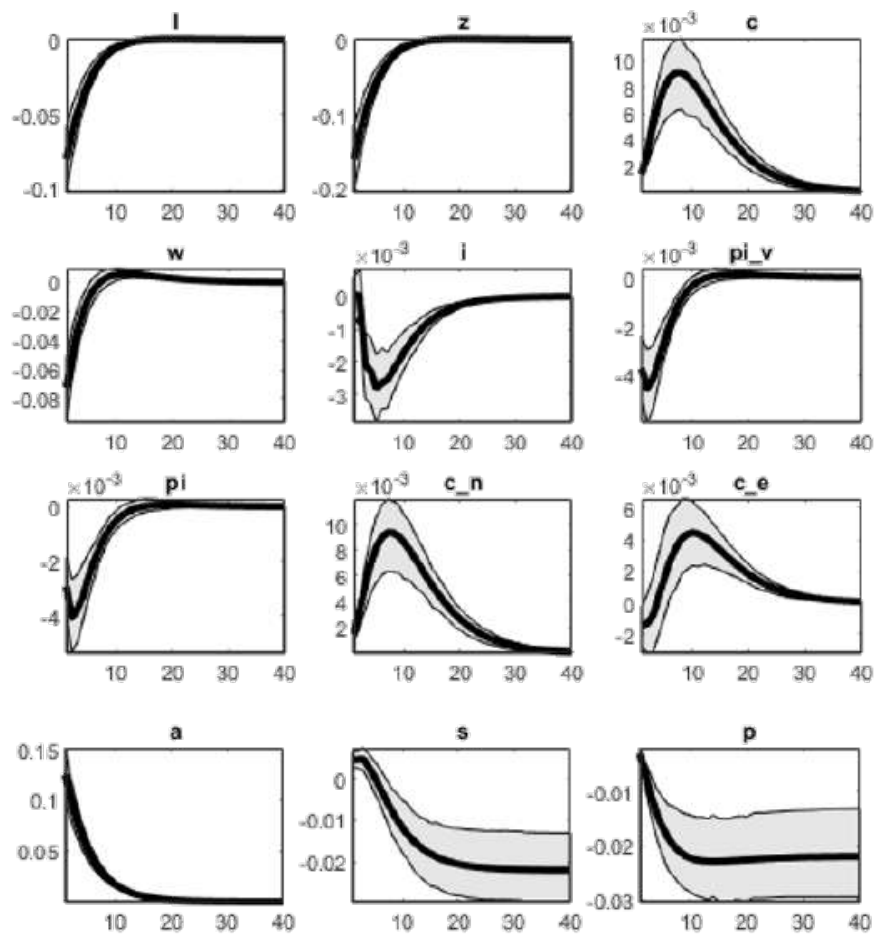

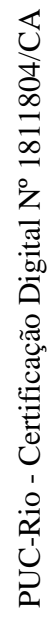
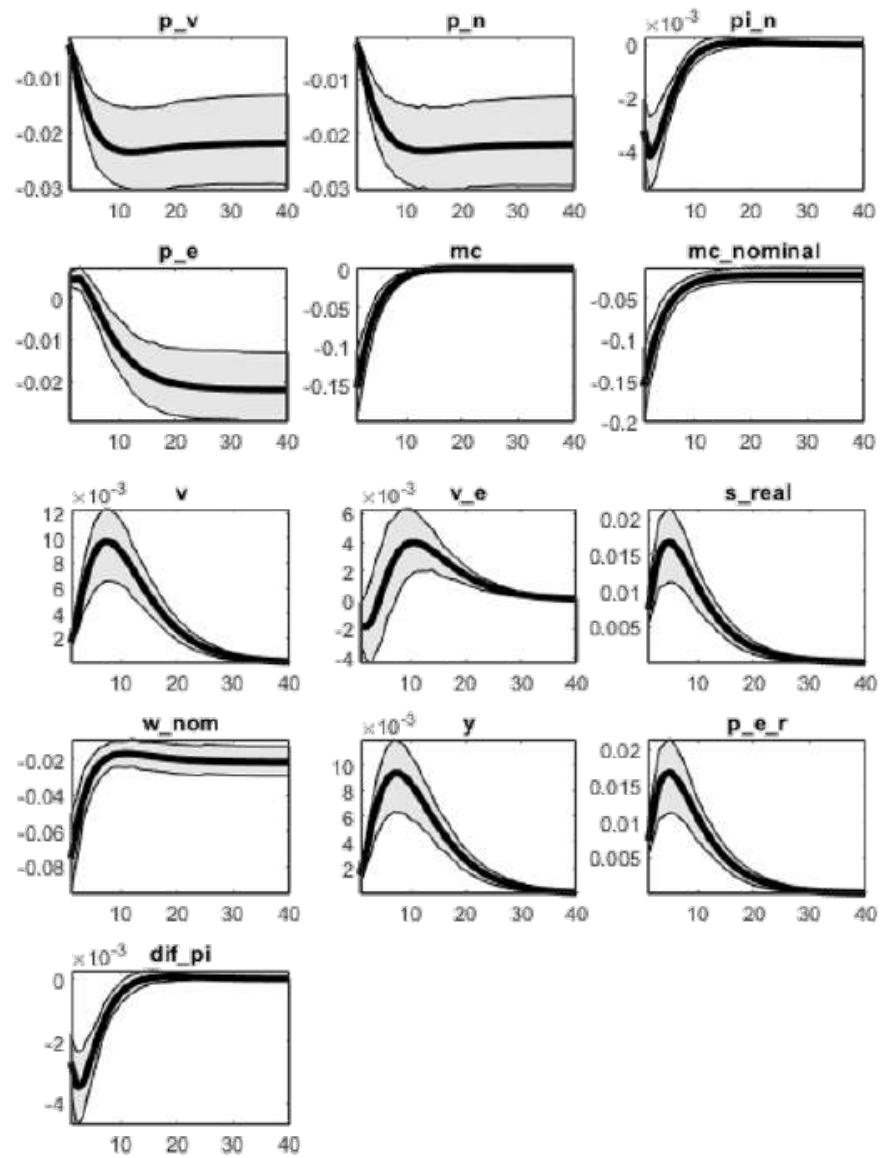
IRF: Choque de prêmio de risco
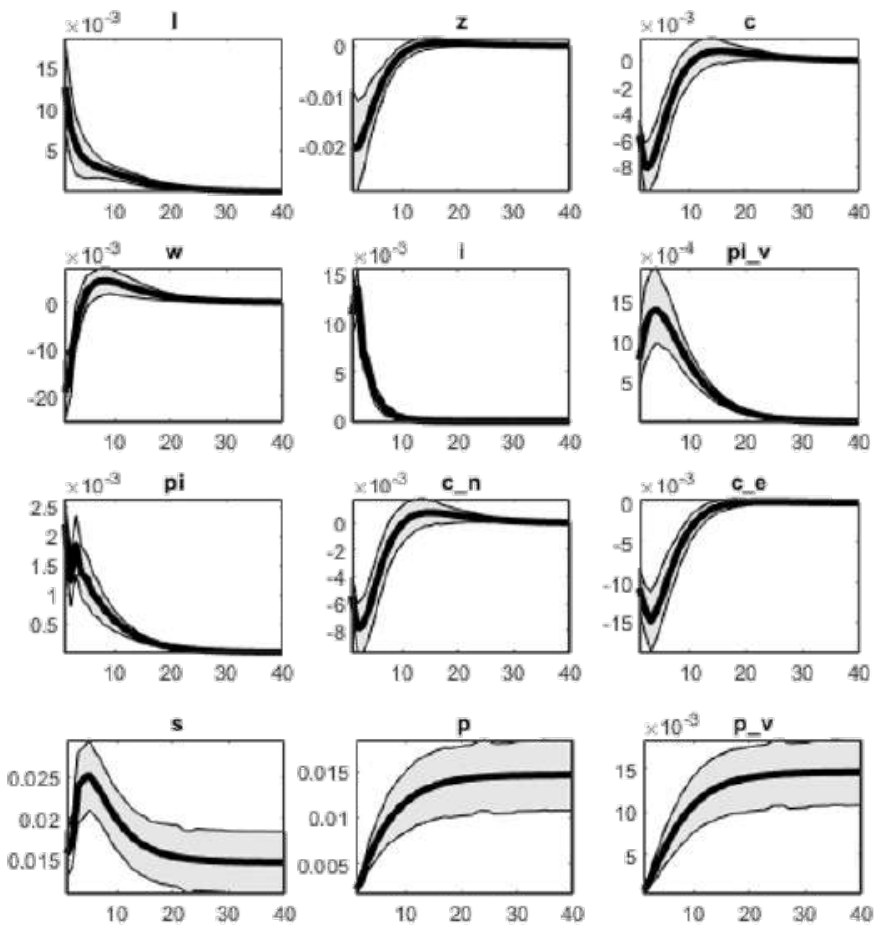

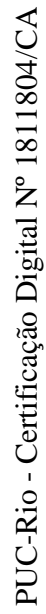
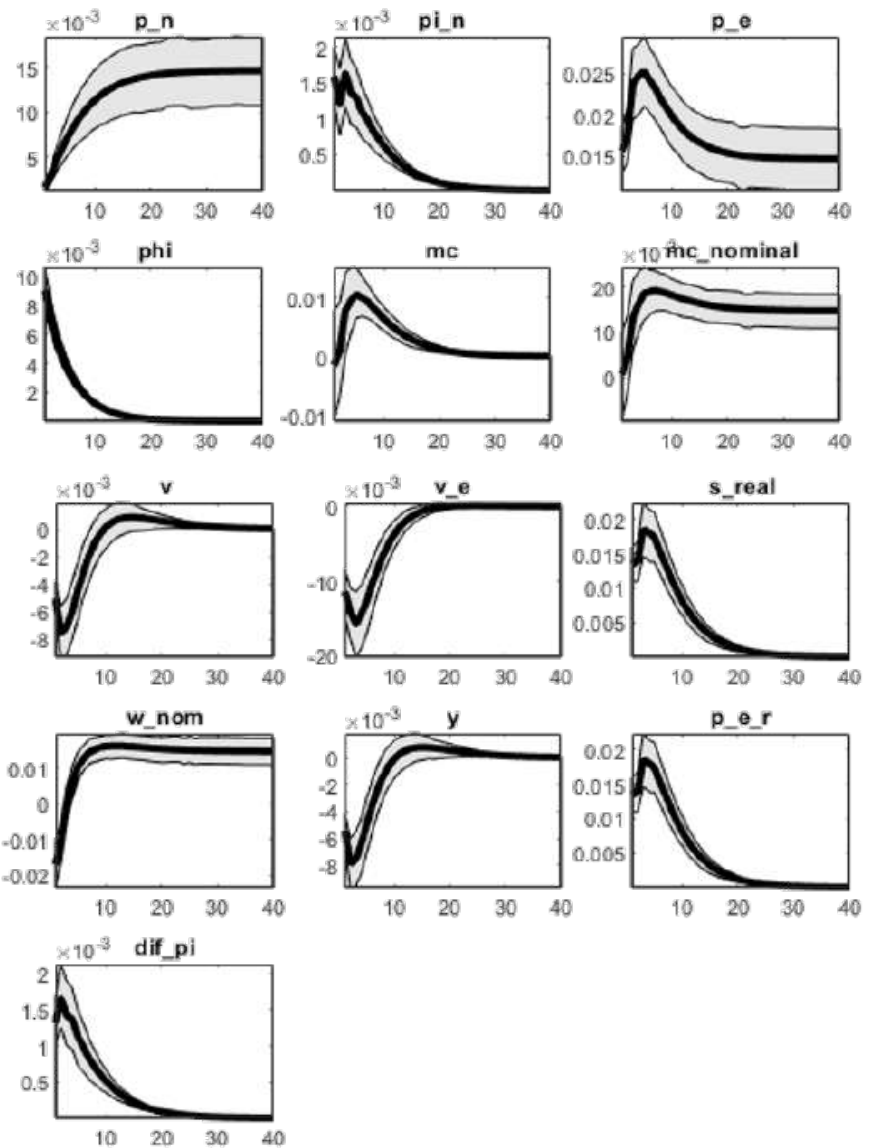
IRF: Choque na taxa de juros estrangeira
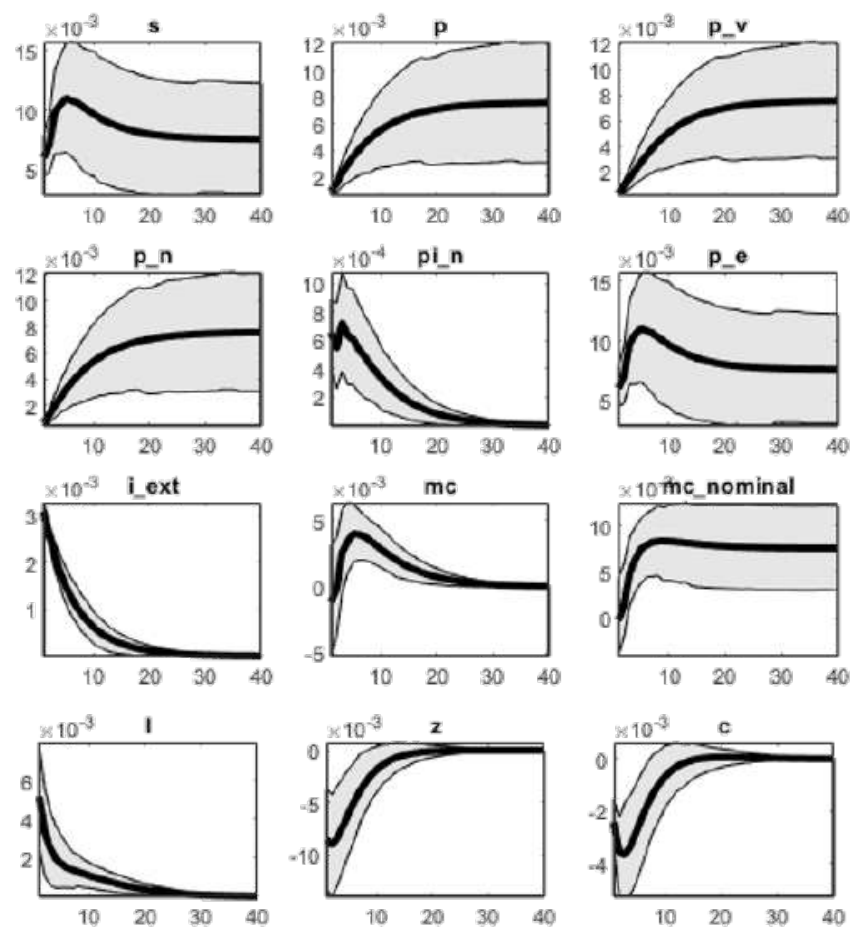

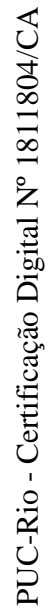
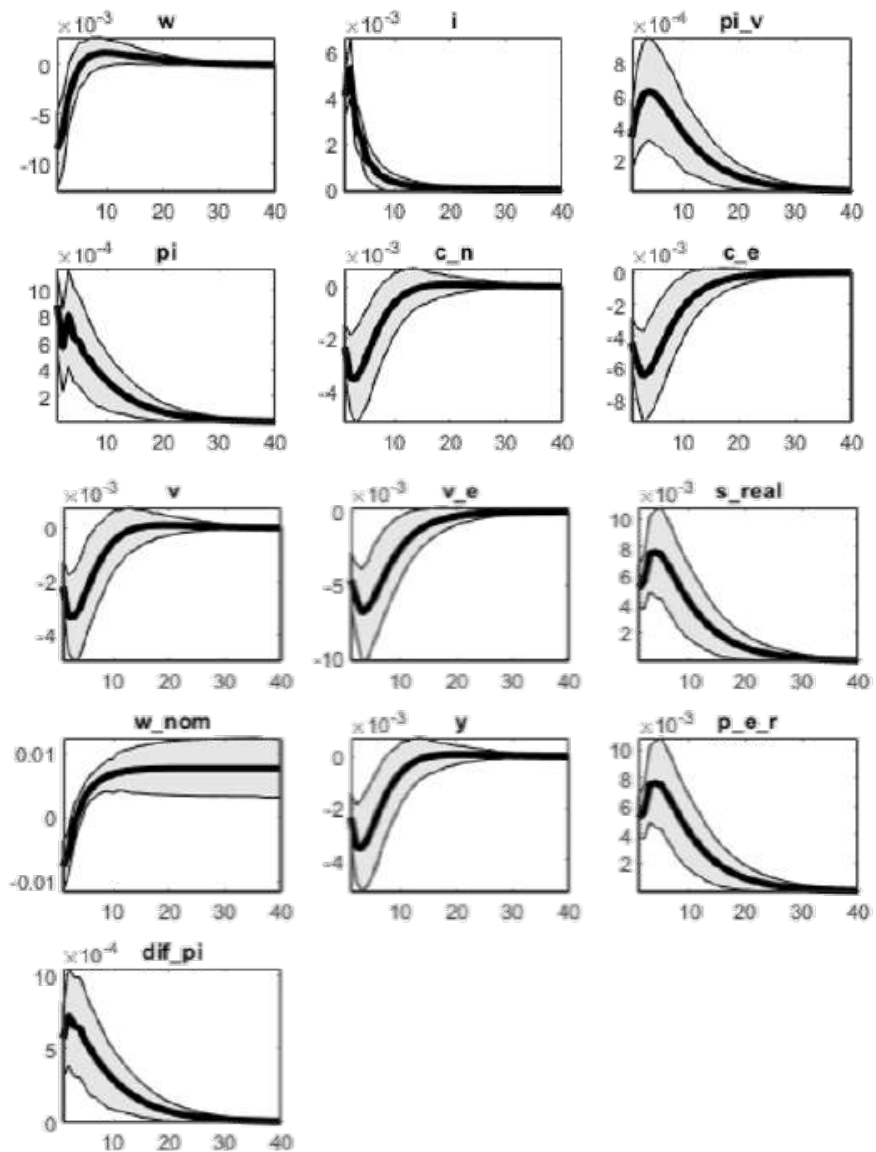